University of Windsor

Scholarship at UWindsor

1992

\title{
Energies and relativistic corrections for the Rydberg states of helium: Variational results and asymptotic analysis
}

Gordon W. F. Drake

University of Windsor

Z. C. Yan

Follow this and additional works at: https://scholar.uwindsor.ca/physicspub

Part of the Physics Commons

\section{Recommended Citation}

Drake, Gordon W. F. and Yan, Z. C.. (1992). Energies and relativistic corrections for the Rydberg states of helium: Variational results and asymptotic analysis. Physical Review A, 46 (5), 2378-2409.

https://scholar.uwindsor.ca/physicspub/75

This Article is brought to you for free and open access by the Department of Physics at Scholarship at UWindsor. It has been accepted for inclusion in Physics Publications by an authorized administrator of Scholarship at UWindsor. For more information, please contact scholarship@uwindsor.ca. 


\title{
Energies and relativistic corrections for the Rydberg states of helium: Variational results and asymptotic analysis
}

\author{
G. W. F. Drake and Zong-Chao Yan \\ Department of Physics, University of Windsor, Windsor, Ontario, Canada N9B 3P4
}

(Received 9 March 1992)

\begin{abstract}
The results of an extended series of high-precision variational calculations for all states of helium up to $n=10$ and $L=7$ (excluding $S$ states above $n=2$ ) are presented. Convergence of the nonrelativistic eigenvalues ranges from five parts in $10^{15}$ for the $2 P$ states to four parts in $10^{19}$ for the $10 \mathrm{~K}$ states. Relativistic and quantum electrodynamic corrections of order $\alpha^{2}, \alpha^{3}, \alpha^{2} \mu / M, \alpha^{2}(\mu / M)^{2}$, and $\alpha^{3} \mu / M$ are included and the required matrix elements listed for each state. For the $1 s 2 p^{3} P_{J}$ states, the lowest-order spindependent matrix elements of the Breit interaction are determined to an accuracy of three parts in $10^{9}$, which, together with higher-order corrections, would be sufficient to allow an improved measurement of the fine-structure constant. Methods of asymptotic analysis are extended to provide improved precision for the relativistic and relativistic-recoil corrections. A comparison with the variational results for the high-angular-momentum states shows that the "standard-atomic-theory" and "long-range-interaction" pictures discussed by Hessels et al. [Phys. Rev. Lett. 65, 2765 (1990)] come into agreement, thereby resolving what appeared to be a discrepancy. The comparison shows that the asymptotic expansions for the total energies are accurate to better than $\pm 100 \mathrm{~Hz}$ for $L>7$, and results are presented for the $9 L$, $10 L$, and $10 M$ states (i.e., angular momentum $L=8$ and 9). Significant discrepancies with experiment persist for transitions among the $n=10$ states, which cannot be easily accommodated by supposed higher-order corrections or additional terms. Finally, the asymptotic analysis indicates that a revision to the quantum-defect method is required for the analysis of high-precision data.
\end{abstract}

PACS number(s): 31.20.Di, 31.15. $+\mathrm{q}, 31.30 . \mathrm{Jv}$

\section{INTRODUCTION}

Transition frequencies among the $n=10$ manifold of states of helium have recently attracted much attention because of the high precision that has been achieved, both experimentally $[1,2]$ and theoretically [3-6]. A comparison between the two appears to show welldefined systematic discrepancies that are much larger than the estimated uncertainties in either. A unique feature of the comparison is that the precision is sufficient to be sensitive to quantum electrodynamic (QED) effects of both the Lamb shift [3-6] and long-range CasimirPolder-retardation types [7-12]. The latter arises (in lowest order) from corrections to short-range approximations made in the usual form of the retarded Breit electron-electron interaction [13]. The result is a form appropriate for low-lying states in the following sense. For a Rydberg electron with radial coordinate $x$ in the range $a_{0}<<x<a_{0} / \alpha$, the retardation terms are proportional to $e^{2} \alpha^{2} a_{0}^{3} / x^{4}$; but for $x \gg a_{0} / \alpha$, the power-law dependence changes to $e^{2} \alpha a_{0}^{4} / x^{5}$. The change in powerlaw dependence is a unique signature of Casimir effects in their many forms [12], but no precise confirmation yet exists. Since the effect can now, in principle, be detected as a residual energy shift in the Rydberg states of helium, it is essential to verify that all other aspects of the theory are correct and under good numerical control.

The experiment of Hessels et al. [1,2] referred to above consists of measuring the frequencies for the sequence of transitions $10 F-10 G, 10 G-10 H, \ldots, 10 K-10 L$. For these transitions, there are two very different theoretical approaches with overlapping ranges of validity, which can be checked against each other. A demonstrated agreement between the two would provide a strong confirmation of both, assuming of course that the underlying formulation of quantum electrodynamics is correct. In historical order, the asymptotic-expansion (AE) method [14-21], valid for high angular momentum $L$, regards the Rydberg electron as moving in the field of a polarizable core consisting of the inner $1 s$ electron and the nucleus with charge $Z$. This gives rise to an asymptotic expansion for the effective nonrelativistic potential experienced by the Rydberg electron of the form

$$
V(x)=-\frac{Z-1}{x}-\frac{\alpha_{1}}{2 x^{4}}-\frac{\alpha_{2}-6 \beta_{1}}{2 x^{6}}-\cdots
$$

together with corresponding asymptotic expansions for the relativistic and other higher-order corrections. Here, $x$ is the radial coordinate for the Rydberg electron, $Z-1$ is the screened nuclear charge, $\alpha_{1}$ is the dipole polarizability of the core, $\alpha_{2}$ is the quadrupole polarizability, and $\beta_{1}$ is a nonadiabatic correction to the dipole term. This method has been developed to a high degree of refinement by Drachman [18-21], with the terms in (1) now being known in their entirety up to $x^{-10}$ [21]. The major limitation of (1) is that the series is an asymptotic one which eventually diverges and so must be terminated after a finite number of terms, depending on the value of $L$. Its great virtue is that the results are entirely analytic 
and cover all high- $L$ states.

The second method, valid for both low- and high- $L$ states, consists of finding high-precision variational solutions to the complete nonrelativistic Schrödinger equation, using correlated basis sets $[22-24,6]$. The relativistic corrections are then determined directly from matrix elements of the Breit interaction, including finite nuclear mass, anomalous magnetic moment, and Lamb-shift corrections. In their presentation of experimental data, Hessels et al. [1] and Lundeen [2] refer to this approach as "standard atomic theory" (SAT). They also introduce a hybrid "long-range-interaction" (LRI) picture in which the nonrelativistic variational eigenvalues are used, together with the $\mathrm{AE}$ results for the relativistic corrections and an approximate version of the Lamb shift. A slight additional complication is that the LRI results also contain the retardation terms and their modifications due to the Casimir-Polder effect [9-11]. However, the shortrange form of the retardation terms is already included in SAT. If the small residual Casimir-Polder modifications, denoted by Au and Mesa [11] as $\Delta V_{\text {ret }}^{\prime \prime}$, are added to SAT, then one would expect it to come into agreement with LRI, at least in the asymptotic limit of high $L$. The results presented by Hessels et al. [1] indicate that they do not agree, with differences of the same order of magnitude as $\Delta V_{\text {ret }}^{\prime \prime}$ itself. Since the primary purpose of their experiment is to observe the effects of $\Delta V_{\text {ret }}^{\prime \prime}$, it is essential to resolve this theoretical discrepancy.

An important result of this paper is to show that if higher-order terms in the asymptotic expansion for the relativistic corrections are included in LRI, and the same expression for the Lamb-shift terms is used, then SAT and LRI come into close agreement for high $L$. What is left is a much larger discrepancy between both theories and experiment for the transition frequencies. However, the main purpose of this paper is to present a detailed listing and asymptotic analysis of high-precision variational calculations to supplement the results already given in Ref. [5]. Except for details of the variational calculations described there and in previous work [22-24], the present paper is reasonably self-contained. In Sec. II, the basic theory of asymptotic expansions is reviewed, and contributions to the nonrelativistic energy discussed. Section III first discusses the relativistic terms of relative order $\alpha^{2} Z^{2}$, and their finite-nuclear-mass (relativisticrecoil) corrections of order $\alpha^{2} Z^{2} \mu / M$; and then extends the asymptotic expansion for these terms to higher order in $1 / x$. Section IV compares the AE results with the variationally determined matrix elements. This section presents a complete tabulation of the necessary matrix elements for all states up to $n=10$ and $1 \leq L \leq 7$, together with the $1 s 2 s^{1} S$ and ${ }^{3} S$ states. A comparison with the AE results for the total energies clearly shows that there is no fundamental difference between SAT and LRI. For low $L$, the differences are due entirely to the lack of convergence in the asymptotic expansions. Section V presents a brief update of the comparison with experiment, especially for transitions among the $n=10$ states. Section VI describes an important modification that should be made to the quantum-defect method due to terms quadratic in the reduced mass ratio $y=\mu / M$. Fi- nally, Sec. VII summarizes the results and presents conclusions.

\section{ASYMPTOTIC EXPANSION THEORY}

The aim of this section is to review asymptoticexpansion theory and the contributions to the nonrelativistic energy, including new terms recently derived by Drachman [21]. Since the effects of mass polarization on both the nonrelativistic energy and the relativistic corrections can be obtained in parallel with little extra effort, these will also be included in both this section and the next.

The basic starting point is the three-particle nonrelativistic Schrödinger equation

$$
\begin{aligned}
\left(-\frac{\hbar^{2}}{2 M} \nabla_{R_{0}}^{2}\right. & -\frac{\hbar^{2}}{2 m} \nabla_{R_{1}}^{2}-\frac{\hbar^{2}}{2 m} \nabla_{R_{2}}^{2}-\frac{Z e^{2}}{\left|\mathbf{R}_{0}-\mathbf{R}_{1}\right|} \\
& \left.-\frac{Z e^{2}}{\left|\mathbf{R}_{0}-\mathbf{R}_{2}\right|}+\frac{e^{2}}{\left|\mathbf{R}_{1}-\mathbf{R}_{2}\right|}\right] \Psi=E_{\mathrm{NR}} \Psi,
\end{aligned}
$$

where $\mathbf{R}_{0}$ is the position vector of the nucleus of mass $M$, and $\mathbf{R}_{1}$ and $\mathbf{R}_{2}$ are the position vectors of the two electrons of mass $m$. The standard transformation to centerof-mass and relative coordinates [25] generates a masspolarization term of the form $-(\mu / M) \nabla_{1} \cdot \nabla_{2}$, where $\mu=m M /(m+M)$ is the reduced mass. This could be treated by perturbation theory; but as pointed out by Drachman [20,21], it is simpler to use instead Jacobi coordinates defined by

$$
\begin{aligned}
& \mathbf{r}=\left(\mathbf{R}_{1}-\mathbf{R}_{0}\right) / a_{\mu} \\
& \mathbf{x}=\Lambda\left[\mathbf{R}_{2}-\mathbf{R}_{0}-\boldsymbol{y}\left(\mathbf{R}_{1}-\mathbf{R}_{0}\right)\right] / a_{\mu}, \\
& \mathbf{X}=\Lambda\left[\mathbf{R}_{0}+\boldsymbol{y}\left(\mathbf{R}_{1}+\mathbf{R}_{2}-\mathbf{R}_{0}\right)\right] / a_{\mu},
\end{aligned}
$$

where

$$
y=\mu / M, \Lambda=1 /\left(1-y^{2}\right),
$$

and $a_{\mu}=(m / \mu) a_{0}$ is the reduced Bohr radius. The derivatives in (2) transform according to

$$
\begin{aligned}
& \nabla_{R_{1}}=a_{\mu}^{-1}\left(\nabla_{r}-\Lambda y \nabla_{x}+\Lambda y \nabla_{X}\right), \\
& \nabla_{R_{2}}=a_{\mu}^{-1}\left(\Lambda \nabla_{x}+\Lambda y \nabla_{X}\right), \\
& \nabla_{R_{0}}=-a_{\mu}^{-1}\left[\nabla_{r}+\Lambda(1-y)\left(\nabla_{x}-\nabla_{X}\right)\right] .
\end{aligned}
$$

Since $X$ is an ignorable coordinate, the Hamiltonian becomes

$$
\begin{aligned}
H & =\left[-\frac{1}{2} \nabla_{r}^{2}-\frac{Z}{r}\right]+\Lambda\left(-\frac{1}{2} \nabla_{x}^{2}-\frac{Z-1}{x}\right]+V(\mathbf{r}, \mathbf{x}) \\
& \equiv h_{r}+\Lambda h_{x}+V(\mathbf{r}, \mathbf{x})
\end{aligned}
$$

with

$V(\mathbf{r}, \mathbf{x})=\Lambda\left(\frac{Z-1}{x}-\frac{Z}{|\mathbf{x}+\Lambda y \mathbf{r}|}+\frac{1}{|\mathbf{x}-\Lambda(1-y) \mathbf{r}|}\right)$

in units of $e^{2} / a_{\mu}$ throughout. In the above, adding and 
subtracting the term $-\Lambda(Z-1) / x$ gives the screened hydrogenic form for $h_{r}+\Lambda h_{x}$, leaving a perturbation $V(\mathbf{r}, \mathbf{x})$ which becomes asymptotically small. Equation (7) immediately implies that the screened hydrogenic energies for a $1 s n L$ configuration are [20]

$$
E_{0}=-Z^{2} / 2-\Lambda(Z-1)^{2} /\left(2 n^{2}\right) .
$$

This differs from the $y=0$ case by

$$
\begin{aligned}
\Delta E_{M}^{(2)} & =-(\Lambda-1)(Z-1)^{2} /\left(2 n^{2}\right) \\
& \simeq-\left(y^{2}+y^{4}\right)(Z-1)^{2} /\left(2 n^{2}\right),
\end{aligned}
$$

which gives in a trivial way the leading term in the second-order mass-polarization correction.

Equation (7) has the important advantage that there is no "mass-polarization" term in the kinetic-energy part, but at the expense of making the potential more complicated. This is not a disadvantage for the polarization model because the multipole expansion of $V(\mathbf{r}, \mathbf{x})$ still has the simple form [20]

$$
V(\mathbf{r}, \mathbf{x})=\frac{1}{x} \sum_{l=1}^{\infty} C_{l}\left(\frac{r}{x}\right)^{l} P_{l}(\hat{\mathbf{r}} \cdot \hat{\mathbf{x}})
$$

with

$$
C_{l}=\frac{(1-y)^{l}-Z(-y)^{l}}{\left(1-y^{2}\right)^{l+1}} .
$$

It is then a simple matter to incorporate the effects of finite mass as follows. Each coefficient in the asymptotic potential (1) is multiplied by combinations of $C_{l}$ factors according to the combinations of multipolarities that contribute. For example, the $2^{l}$-pole polarizability $\alpha_{l}$ is quadratic in $C_{l}$, and so is replaced by $C_{l}^{2} \alpha_{l}$. The same is true of $\beta_{l}$. Provided that $\mu / M$ is small, the $C_{l}$ factors can be expanded according to

$$
\begin{aligned}
& C_{1}=1+(Z-1) y+2 y^{2}+\cdots, \\
& C_{2}=1-2 y+(4-Z) y^{2}+\cdots, \\
& C_{3}=1-3 y+7 y^{2}+\cdots, \\
& C_{4}=1-4 y+11 y^{2}+\cdots .
\end{aligned}
$$

Thus, for example, the leading term $-\alpha_{1}\left\langle x^{-4}\right\rangle_{n L} / 2$ in the asymptotic potential (1) becomes $-\alpha_{1} C_{1}^{2}\left\langle x^{-4}\right\rangle_{n L} / 2$ because $\alpha_{1}$ involves two dipole interactions. The difference $-\alpha_{1}\left(C_{1}^{2}-1\right)\left\langle x^{-4}\right\rangle_{n L} / 2$, together with Eq. (10), are the leading terms in the mass-polarization correction to the energy. The expansions in (13) allow the first- and second-order contributions to the corresponding mass-polarization energy coefficients $\varepsilon_{M}^{(1)}$ and $\varepsilon_{M}^{(2)}$ to be separated. Terms of order $\left\langle x^{-9}\right\rangle_{n L}$ and $\left\langle x^{-10}\right\rangle_{n L}$ have recently been evaluated by Drachman [21], and additional nonadiabatic corrections by Drake [26]. Combining these and separating the mass dependence, the result is

$$
\begin{aligned}
\varepsilon_{M}^{(1)}= & -(Z-1) \alpha_{1}\left\langle x^{-4}\right\rangle_{n L}+\left[2 \alpha_{2}+6(Z-1) \beta_{1}\right]\left\langle x^{-6}\right\rangle_{n L}+\left[(Z-2) \delta+\frac{16}{5}(Z-1)^{2} \gamma\right]\left\langle x^{-7}\right\rangle_{n L} \\
& +\left\{3 \alpha_{3}-30 \beta_{2}+2(Z-1)\left(\alpha_{1} \beta_{1}-\epsilon\right)-72(Z-1) \gamma[1+L(L+1) / 10]\right\}\left\langle x^{-8}\right\rangle_{n L}+\frac{1}{2} c_{9}^{(1)}\left\langle x^{-9}\right\rangle_{n L} \\
& +\frac{1}{2} c_{10}^{(1)}\left\langle x^{-10}\right\rangle_{n L}+4(Z-1) e_{2,0}^{(1,1)}+2\left[(Z-3)-12(Z-1) \beta_{1} / \alpha_{2}\right] e_{2,0}^{(1,2)}, \\
\varepsilon_{M}^{(2)}= & -\frac{1}{2}(Z-1)^{2} n^{-2}-\frac{1}{2}[5+Z(Z-2)] \alpha_{1}\left\langle x^{-4}\right\rangle_{n L}-\left\{(6-Z) \alpha_{2}-3[5+Z(Z-2)] \beta_{1}\right\}\left\langle x^{-6}\right\rangle_{n L} \\
& +\left\{\frac{1}{2}[3+(Z-2)(Z-5)] \delta+\frac{8}{5}(Z-1)[5+Z(Z-2)]\right\}\left\langle x^{-7}\right\rangle_{n L} \\
& +\left\{-\frac{23}{2} \alpha_{3}+15(6-Z) \beta_{2}+[7+3 Z(Z-2)]\left(\alpha_{1} \beta_{1}-\epsilon\right)\right. \\
& -36[5+Z(Z-2)] \gamma[1+L(L+1) / 10]\}\left\langle x^{-8}\right\rangle_{n L}+2[7+3(Z-2)] e_{2,0}^{(1,1)}
\end{aligned}
$$

where

$e_{2,0}^{(j, k)}=-\frac{1}{2} \alpha_{j} \alpha_{k}\left(2-\delta_{j, k}\right)\left\langle\phi_{1}^{(2 j+2)}\left|x^{-2 k-2}\right| \phi_{0}\right\rangle_{n L}$

is a second-order adiabatic polarization energy in which $\phi_{1}^{(i)}$ satisfies a first-order hydrogenic perturbation equation for the Rydberg electron with $-1 /\left(2 x^{i}\right)$ as the perturbation. Closed-form analytic expressions are known for $e_{2,0}^{(1,1)}$ and $e_{2,0}^{(1,2)}[27,28]$, and numerical values are tabulated by Drachman [19,21]. Numerical values for the quantities $\alpha_{i}, \beta_{i}, \gamma, \delta$, and $\epsilon$ in Eqs. (14) and (15) are listed by Drachman [18], and by Drake and Swainson [27]. The $c_{j}^{(1)}$ in Eq. (14) represent collections of several coefficients $[21,26]$ multiplied by the terms linear in $y$ in
Eq. (13). The final result is

$$
\begin{gathered}
c_{9}^{(1)}=-Z^{-10}\left[\frac{7581}{4}+\frac{80637}{14}(Z-1)+\frac{493323}{252}(Z-1)^{2}\right], \\
c_{10}^{(1)}=Z^{-12}\left[31422+\frac{26677}{32}(Z-1)\right. \\
\left.\quad+\frac{71445}{4} Z^{2}+\frac{48365}{2}(Z-1) Z^{2}\right] \\
+Z^{-10} L(L+1)\left[\frac{35985}{42}+\frac{145095}{28}(Z-1)\right] .
\end{gathered}
$$

Similar results can be obtained for $c_{9}^{(2)}$ and $c_{10}^{(2)}$ [26] and a contribution $\frac{1}{2} c_{9}^{(2)}\left\langle x^{-9}\right\rangle_{n L}+\frac{1}{2} c_{10}^{(2)}\left\langle x^{-10}\right\rangle_{n L}$ added to Eq. (15) for $\varepsilon_{M}^{(2)}$, but the change is too small to be impor- 
tant. The asymptotic mass-polarization correction to the energy is then

$$
\Delta E_{\mathrm{mp}}=y \varepsilon_{M}^{(1)}+y^{2} \varepsilon_{M}^{(2)}+\cdots
$$

Detailed numerical comparisons with variational calculations for the Rydberg states of helium will be discussed in Sec. IV A. Note that $y^{2} \varepsilon_{M}^{(2)}$ eventually becomes larger than $y \varepsilon_{M}^{(1)}$ because of the $n^{-2}$ term in (15). However, this does not indicate poor convergence, it merely indicates an even-odd alternation in the magnitudes of the terms.

\section{RELATIVISTIC CORRECTIONS}

\section{A. Standard formulation}

This section reviews the standard formulation for the Breit interaction operators and their finite-mass corrections, expressed in terms of conventional coordinates $\mathbf{r}_{1}$ and $\mathbf{r}_{2}$ for the positions of the two electrons relative to the nucleus. Starting from the Dirac Hamiltonian summed over particles, and the Breit interaction summed over all pairwise interactions, the terms in the center-ofmass frame are [25,29] (in units of $e^{2} / a_{\mu}$ )

$H_{1}=-\frac{\alpha^{2}}{8}\left[\frac{\mu}{m}\right)^{3}\left(\nabla_{1}^{4}+\nabla_{2}^{4}\right)$,

$H_{3}=\frac{\alpha^{2}}{2}\left(\frac{\mu}{m}\right)^{2}\left[\left(\nabla_{1} V \times \mathbf{p}_{1}\right) \cdot \mathbf{s}_{1}+\left(\nabla_{2} V \times \mathbf{p}_{2}\right) \cdot \mathbf{s}_{2}\right]$,

$H_{4}=\frac{\alpha^{2}}{4}\left(\frac{\mu}{m}\right)^{2}\left(\nabla_{1}^{2} V+\nabla_{1} V \cdot \nabla_{1}+\nabla_{2}^{2} V+\nabla_{2} V \cdot \nabla_{2}\right)$,

$\left.M_{2}=\frac{\alpha^{2}}{2}\left(\frac{\mu}{m}\right)^{2}\left(\frac{1}{r_{12}} \nabla_{1} \cdot \nabla_{2}+\frac{1}{r_{12}^{3}} \mathbf{r}_{12} \cdot\left(\mathbf{r}_{12} \cdot \nabla_{1}\right) \nabla_{2}\right)+\frac{Z \alpha^{2}}{2} \int \frac{\mu}{M}\right)\left(\frac{\mu}{m}\right)^{2}\left(\frac{1}{r_{1}}\left(\nabla_{1}+\nabla_{2}\right) \cdot \nabla_{1}+\frac{1}{r_{1}^{3}} \mathbf{r}_{1} \cdot\left[\mathbf{r}_{1} \cdot\left(\nabla_{1}+\nabla_{2}\right)\right] \nabla_{1}\right)$

$+\frac{Z \alpha^{2}}{2}\left(\frac{\mu}{M}\right)\left(\frac{\mu}{m}\right)^{2}\left[\frac{1}{r_{2}}\left(\nabla_{1}+\nabla_{2}\right) \cdot \nabla_{2}+\frac{1}{r_{2}^{3}} \mathbf{r}_{2} \cdot\left[\mathbf{r}_{2} \cdot\left(\nabla_{1}+\nabla_{2}\right)\right] \nabla_{2}\right)$,

$M_{3}=-\frac{1}{2} g_{e} \alpha^{2}\left(\frac{\mu}{m}\right)^{2}\left\{\left[\left(\nabla_{1} r_{12}^{-1}\right) \times \mathbf{p}_{2}\right] \cdot \mathbf{s}_{1}+\left[\left(\nabla_{2} r_{12}^{-1}\right) \times \mathbf{p}_{1}\right] \cdot \mathbf{s}_{2}\right\}$

$-\frac{1}{2} g_{e} Z \alpha^{2}\left[\frac{\mu}{M}\right]\left[\frac{\mu}{m}\right]^{2}\left\{\left[\left(\nabla_{1} r_{1}^{-1}\right) \times\left(\mathbf{p}_{1}+\mathbf{p}_{2}\right)\right] \cdot \mathbf{s}_{1}+\left[\left(\nabla_{2} r_{2}^{-1}\right) \times\left(\mathbf{p}_{1}+\mathbf{p}_{2}\right)\right] \cdot \mathbf{s}_{2}\right\}$,

$M_{3}^{\prime}=-\gamma_{e} Z \alpha^{2}\left[\frac{\mu}{m}\right]^{2}\left\{\left[\left(\nabla_{1} r_{1}^{-1}\right) \times \mathbf{p}_{1}\right] \cdot \mathbf{s}_{1}+\left[\left(\nabla_{2} r_{2}^{-1}\right) \times \mathbf{p}_{2}\right] \cdot \mathbf{s}_{2}\right\}+\gamma_{e} \alpha^{2}\left[\frac{\mu}{m}\right]^{2}\left\{\left[\left(\nabla_{1} r_{12}^{-1}\right) \times \mathbf{p}_{1}\right] \cdot \mathbf{s}_{1}+\left[\left(\nabla_{2} r_{12}^{-1}\right) \times \mathbf{p}_{2}\right] \cdot \mathbf{s}_{2}\right\}$,

$M_{5}=\frac{1}{4} g_{e}^{2} \alpha^{2}\left(\frac{\mu}{m}\right)^{2} \nabla_{1} \cdot\left[\left(\nabla_{2} r_{12}^{-1}\right) \cdot \mathbf{s}_{1}\right] \mathbf{s}_{2}$,

$M_{6}=-\frac{8}{3} \pi \alpha^{2}\left(\frac{\mu}{m}\right)^{2} \delta\left(\mathbf{r}_{12}\right) \mathbf{s}_{1} \cdot \mathbf{s}_{2}$,

where

$$
V=-Z / r_{1}-Z / r_{2}+1 / r_{12} .
$$

$\gamma_{e} \simeq \alpha / 2 \pi$ is the electron anomalous magnetic moment, $g_{e}=2\left(1+\gamma_{e}\right)$, and $r_{12}=\left|\mathbf{r}_{1}-\mathbf{r}_{2}\right|$. The $H_{i}$ are the Dirac terms and the $\boldsymbol{M}_{i}$ are the Breit interaction terms, numbered in accordance with the convention of the Bethe and Salpeter [25]. The terms are written out in detail because the origin of all the finite-mass corrections is not evident from the derivation of Bethe and Salpeter.

For purposes of numerical calculation, it is usual to transform together the two terms $H_{1}+H_{4}$ to a form in which the singular matrix elements $\left(\Psi, \nabla_{1}^{4} \Psi\right)+\left(\Psi, \nabla_{2}^{4} \Psi\right)$ are replaced by the less singular form $\left(\nabla_{2}^{2} \Psi, \nabla_{1}^{2} \Psi\right)[25,30]$. With the use of arguments similar to those of Bethe and Salpeter [25], the result for the finite-nuclear-mass case is $[5,24]$

$$
\begin{aligned}
\left\langle H_{1}+H_{4}\right\rangle= & -\frac{\alpha^{2}}{4}\left[\frac{\mu}{m}\right]^{3}\left\langle 2 f^{2}-4 y f \mathbf{p}_{1} \cdot \mathbf{p}_{2}\right. \\
& \left.+2\left(y \mathbf{p}_{1} \cdot \mathbf{p}_{2}\right)^{2}-p_{1}^{2} p_{2}^{2}\right\rangle \\
& +\alpha^{2} \pi\left(\frac{\mu}{m}\right]^{2}\left[Z\left\langle\delta\left(\mathbf{r}_{1}\right)\right\rangle-\left\langle\delta\left(\mathbf{r}_{12}\right)\right\rangle\right],
\end{aligned}
$$

where 


$$
\begin{aligned}
f & =E-V \\
& =E+Z / r_{1}+Z / r_{2}-1 / r_{12} .
\end{aligned}
$$

The $-4 y f \mathbf{p}_{1} \cdot \mathbf{p}_{2}+2\left(y \mathbf{p}_{1} \cdot \mathbf{p}_{2}\right)^{2}$ terms are additional contributions which do not appear in standard treatments. Only the first part linear in $y$ is included in the variational results presented previously $[3-5,22-24]$. The very small correction of order $\alpha^{2} y^{2}$ from the second part is further discussed in Sec. IV B in terms of its asymptotic expansion.

To facilitate a systematic presentation of the results, the terms $H_{1}$ to $H_{4}$ and $M_{2}$ to $M_{6}$ are now separated into terms of lowest order $\alpha^{2}$, relativistic reduced-mass corrections of order $\alpha^{2} y$, and anomalous magneticmoment corrections of order $\alpha^{3}$. Collecting terms of similar type, the lowest-order Pauli form of the Breit interaction is

$$
\begin{aligned}
& B_{1}=-\frac{\alpha^{2}}{8}\left(\nabla_{1}^{4}+\nabla_{2}^{4}\right), \\
& B_{2}=\frac{\alpha^{2}}{2}\left(\frac{1}{r_{12}} \nabla_{1} \cdot \nabla_{2}+\frac{1}{r_{12}^{3}} \mathbf{r}_{12} \cdot\left(\mathbf{r}_{12} \cdot \nabla_{1}\right) \nabla_{2}\right), \\
& B_{4}=\alpha^{2} \pi\left(\frac{Z}{2} \delta\left(\mathbf{r}_{1}\right)+\frac{Z}{2} \delta\left(\mathbf{r}_{2}\right)-\delta\left(\mathbf{r}_{12}\right)\right] .
\end{aligned}
$$

The spin-dependent terms are

$$
B_{3}=B_{3, Z}+B_{3, e}
$$

with

$$
\begin{aligned}
& B_{3, Z}=\frac{Z \alpha^{2}}{2} {\left[\frac{1}{r_{1}^{3}}\left(\mathbf{r}_{1} \times \mathbf{p}_{1}\right) \cdot \mathbf{s}_{1}+\frac{1}{r_{2}^{3}}\left(\mathbf{r}_{2} \times \mathbf{p}_{2}\right) \cdot \mathbf{s}_{2}\right], } \\
& B_{3, e}=\frac{\alpha^{2}}{2 r_{12}^{3}}\left\{\left[\left(\mathbf{r}_{2}-\mathbf{r}_{1}\right) \times \mathbf{p}_{1}\right] \cdot\left(\mathbf{s}_{1}+2 \mathbf{s}_{2}\right)\right. \\
&\left.+\left[\left(\mathbf{r}_{1}-\mathbf{r}_{2}\right) \times \mathbf{p}_{2}\right] \cdot\left(\mathbf{s}_{2}+2 \mathbf{s}_{1}\right)\right\},
\end{aligned}
$$

and

$$
\begin{aligned}
\boldsymbol{B}_{5} & =\alpha^{2} \nabla_{1} \cdot\left[\left(\boldsymbol{\nabla}_{2} r_{12}^{-1}\right) \cdot \mathbf{s}_{1}\right] \mathbf{s}_{2} \\
& =\alpha^{2}\left[\frac{1}{r_{12}^{3}} \mathbf{s}_{1} \cdot \mathbf{s}_{2}-\frac{3}{r_{12}^{5}}\left(\mathbf{s}_{1} \cdot \mathbf{r}_{12}\right)\left(\mathbf{s}_{2} \cdot \mathbf{r}_{12}\right)\right], \\
\boldsymbol{B}_{6} & =-\frac{8}{3} \pi \alpha^{2} \delta\left(\mathbf{r}_{12}\right) \mathbf{s}_{1} \cdot \mathbf{s}_{2},
\end{aligned}
$$

all in units of $e^{2} / a_{\mu}$, and expectation values are assumed with respect to $\Psi_{\infty}$ satisfying the nonrelativistic Schrödinger equation for infinite nuclear mass. $B_{5}$ vanishes for singlet states because the operator can be written as the tensor product of orbital and spin parts of rank two. Because of the $\delta\left(\mathbf{r}_{12}\right)$ factor, $\boldsymbol{B}_{6}$ only contributes for singlet states, where

$$
\left\langle\mathbf{s}_{1} \cdot \mathbf{s}_{2}\right\rangle=\frac{1}{2}\left\langle S^{2}-s_{1}^{2}-s_{2}^{2}\right\rangle=-\frac{3}{4} .
$$

In summary, the lowest-order relativistic correction is

$$
\begin{aligned}
\Delta E_{\mathrm{rel}}= & \left\langle B_{1}\right\rangle+\left\langle B_{2}\right\rangle+\left\langle B_{3}\right\rangle+\left\langle B_{5}\right\rangle \\
& +\pi \alpha^{2}\left\langle Z \delta\left(r_{1}\right)+\delta\left(r_{12}\right)\right\rangle,
\end{aligned}
$$

where the contributions involving $\delta$ functions in $B_{4}$ and $B_{6}$ have been combined to give the last term.

The finite-mass corrections can now be simply expressed in terms of the above. With the use of

$$
\left(\frac{\mu}{m}\right)^{n} \simeq 1-n y
$$

in Eqs. (20) - (27), the results are

$$
\begin{aligned}
& B_{1}^{M}=-3 y B_{1}, \\
& B_{2}^{M}=-2 y B_{2}+\Delta_{2}, \\
& B_{3}^{M}=-2 y B_{3, e}+\Delta_{3}, \\
& B_{4}^{M}=-2 y B_{4}, \\
& B_{5}^{M}=-2 y B_{5}, \\
& B_{6}^{M}=-2 y B_{6},
\end{aligned}
$$

where, from (23) and (24),

$$
\begin{aligned}
\Delta_{2}= & \frac{1}{2} Z \alpha^{2} y\left(\frac{1}{r_{1}}\left(\nabla_{1}+\nabla_{2}\right) \cdot \nabla_{1}+\frac{1}{r_{1}^{3}} \mathbf{r}_{1} \cdot\left[\mathbf{r}_{1} \cdot\left(\nabla_{1}+\nabla_{2}\right)\right] \nabla_{1}\right) \\
+\frac{1}{2} Z \alpha^{2} y & \left(\frac{1}{r_{2}}\left(\nabla_{1}+\nabla_{2}\right) \cdot \nabla_{2}\right. \\
& \left.+\frac{1}{r_{2}^{3}} \mathbf{r}_{2} \cdot\left[\mathbf{r}_{2} \cdot\left(\nabla_{1}+\nabla_{2}\right)\right] \nabla_{2}\right)
\end{aligned}
$$

$\Delta_{3}=Z \alpha^{2} y\left[\frac{1}{r_{1}^{3}}\left(\mathbf{r}_{1} \times \mathbf{p}_{2}\right) \cdot \mathbf{s}_{1}+\frac{1}{r_{2}^{3}}\left(\mathbf{r}_{2} \times \mathbf{p}_{1}\right) \cdot \mathbf{s}_{2}\right]$.

Notice that the term $-2 y B_{3, Z}$ that would otherwise appear in Eq. (43) cancels with similar terms that would otherwise appear in the definition of $\Delta_{3}$; i.e., $\left(\mathbf{r}_{1} \times \mathbf{p}_{1}\right) \cdot \mathbf{s}_{1}$ and $\left(\mathbf{r}_{2} \times \mathbf{p}_{2}\right) \cdot \mathbf{s}_{2}$ terms. $\left(\Delta_{3}\right.$ corresponds to $\Delta_{1}$ of Stone [29] and our previous work. The notation has been changed to emphasize the connection with $B_{3}$.) The total relativistic-recoil correction due to the explicit reducedmass dependence of the Breit interaction is

$$
\left(\Delta E_{\mathrm{RR}}\right)_{M}=\sum_{i=1}^{6}\left\langle B_{i}^{M}\right\rangle \text {. }
$$

The above correction reduces to a well-known result in the one-electron case. In this limit, $\Delta_{3}$ does not contribute and $\Delta_{2}$ reduces to

$$
\Delta_{2}=\frac{Z \alpha^{2}}{2 r} y\left(\nabla^{2}+\nabla_{r}^{2}\right)
$$

where $\nabla_{r}^{2}=r^{-2} \mathbf{r} \cdot(\mathbf{r} \cdot \nabla) \nabla$. This term, when combined with the others in Eq. (49), gives the operator

$$
H_{b}=\alpha^{2} y\left[\frac{3}{8} \nabla^{4}+\frac{Z}{2 r}\left(\nabla^{2}+\nabla_{r}^{2}\right)+\frac{1}{2} Z \nabla^{2}\left(r^{-1}\right)\right] \text {. }
$$

As shown by Bethe and Salpeter [25] (see Eq. 42.7, p. 195), the expectation value of $H_{b}$ reduces to the oneelectron relativistic reduced-mass shift 


$$
E_{b}=-\left(\frac{Z \alpha}{2 n}\right)^{2} y\left|E_{\mathrm{NR}}\right|,
$$

where $E_{\mathrm{NR}}=-1 /\left(2 n^{2}\right)$. This is in addition to the mass shift already contained implicitly in Eq. (39) due to the use of the reduced-mass Rydberg. A corresponding limit can be expected for Rydberg states of two-electron atoms (see Sec. III B 2).

Second-order cross terms with the mass-polarization operator produce further corrections of order $\alpha^{2} y$ in the two-electron case. Denoting these relativistic recoil terms by $B_{i}^{\mathrm{X}}(i=1, \ldots, 6)$, they can each be expressed in the form

$$
\begin{aligned}
B_{i}^{\mathrm{X}} & =2\left\langle\delta \Psi_{\infty}\left|B_{i}\right| \Psi_{\infty}\right\rangle \\
& =2 y \sum_{k \neq 0} \frac{\left\langle\Psi_{\infty}\left|\mathrm{p}_{1} \cdot \mathrm{p}_{2}\right| k\right\rangle\left\langle k\left|B_{i}\right| \Psi_{\infty}\right\rangle}{E_{\infty}(0)-E_{\infty}(k)},
\end{aligned}
$$

where $k=0$ denotes the unperturbed state $\Psi_{\infty}$, and $\delta \Psi_{\infty}$ is the perturbation due to mass polarization. The perturbation sum can be calculated explicitly by solving a firstorder perturbation equation, as done by Lewis and Serafino [31], or implicitly by recalculating the matrix elements of the $B_{i}$ with respect to the $\Psi_{M}$ solutions of the finite-mass Schrödinger equation (i.e., with mass polarization included) and writing

$$
\begin{aligned}
B_{i}^{\mathrm{X}} \simeq & \left\langle\Psi_{M}\left|B_{i}\right| \Psi_{M}\right\rangle-\left\langle\Psi_{\infty}\left|B_{i}\right| \Psi_{\infty}\right\rangle \\
& +\alpha^{2} y\left\langle\Psi_{M}\left|f \mathrm{p}_{1} \cdot \mathrm{p}_{2}\right| \Psi_{M}\right\rangle \delta_{i, 1}
\end{aligned}
$$

The last term is the $y=\mu / M$ correction in (28) for $i=1$. This procedure automatically includes higher-order terms in the $(\mu / M) \mathbf{p}_{1} \cdot \mathbf{p}_{2}$ perturbation series, but since $\mu / M$ is small, it gives the coefficient of the $\alpha^{2} \mu / M$ cross term to sufficient accuracy that isotope shifts can be calculated without redoing the calculations for each nuclear mass. However, higher-order terms quadratic in $\mu / M$ affect the comparison with asymptotic expansions, as further discussed in Sec. IV B. The total correction due to second-order cross terms is

$$
\left(\Delta E_{\mathrm{RR}}\right)_{\mathrm{X}}=\sum_{i=1}^{6}\left\langle B_{i}^{\mathrm{X}}\right\rangle \text {. }
$$

The spin-dependent anomalous magnetic moment corrections only affect $B_{3}$ and $B_{5}$. The terms are

$$
\begin{aligned}
& B_{3, Z}^{A}=2 \gamma_{e} B_{3, Z}, \\
& B_{3, e}^{A}=2 \gamma_{e} \frac{\alpha^{2}}{2 r_{12}^{3}}\left\{\left[\left(\mathbf{r}_{2}-\mathbf{r}_{1}\right) \times \mathbf{p}_{1}\right] \cdot\left(\mathbf{s}_{1}+\mathbf{s}_{2}\right)\right. \\
& \left.+\left[\left(\mathbf{r}_{1}-\mathbf{r}_{2}\right) \times \mathbf{p}_{2}\right] \cdot\left(\mathbf{s}_{2}+\mathbf{s}_{1}\right)\right\}, \\
& B_{5}^{A}=2 \gamma_{e} B_{5} .
\end{aligned}
$$

Comparing with Eq. (35) and using

$$
\mathbf{s}_{1}+\mathbf{s}_{2}=\frac{2}{3}\left(\mathbf{s}_{1}+2 \mathbf{s}_{2}\right)+\frac{1}{3}\left(\mathbf{s}_{1}-\mathbf{s}_{2}\right),
$$

it can be seen that the matrix elements of $B_{3, e}^{A}$ between states with different total spin $\mathbf{S}=\mathbf{s}_{1}+\mathbf{s}_{2}$ are

$$
\left\langle a^{\prime} S^{\prime}\left|B_{3, e}^{A}\right| a S\right\rangle=\frac{4}{3} \gamma_{e} \delta_{S, S^{\prime}}\left\langle a^{\prime} S^{\prime}\left|B_{3, e}\right| a S\right\rangle,
$$

and the right-hand side is zero if $S=0$. Thus, only the diagonal matrix elements are nonvanishing in $L S$ coupling, and only then for triplet states. The total anomalous magnetic-moment matrix elements in $L S$ coupling are thus

$$
\begin{aligned}
& \left\langle a^{\prime} S^{\prime}\left|\Delta H_{\text {anom }}\right| a S\right\rangle \\
& \quad=2 \gamma_{e}\left\langle a^{\prime} S^{\prime}\left|B_{3, Z}+\frac{2}{3} \delta_{S, S^{\prime}} B_{3, e}+B_{5}\right| a S\right\rangle
\end{aligned}
$$

excluding $\delta$-function terms. For consistency, these will be included along with other QED corrections of the same order [see Eqs. (118) and (134) to follow]. The corresponding energy change is $\Delta E_{\text {anom }}$.

\section{B. Asymptotic expansions}

This section applies the asymptotic expansion methods introduced in Sec. II to the calculation of the relativistic terms contained in the Breit interaction and their finitemass corrections. The results substantially extend the known terms due to the one-electron Dirac energy and relativistic polarizability discussed previously by Drachman [19].

\section{Spin-independent terms}

Consider first the spin-independent parts of the Breit interaction. As discussed, for example, by Drachman [19] and Drake [5], the asymptotic limit for $\left\langle B_{1}+B_{4}\right\rangle$ is (in units of $e^{2} / a_{\mu}$ throughout)

$$
\left\langle B_{1}+B_{4}\right\rangle \rightarrow-\alpha^{2} Z^{4} / 8+h_{1}(n L)+\Delta B_{1}\left(\alpha_{\mathrm{rel}}\right)+\Delta B_{1}\left(\phi_{1}\right),
$$

where

$$
\begin{aligned}
& h_{1}(n L)=\frac{\alpha^{2}(Z-1)^{4}}{2 n^{3}}\left[\frac{3}{4 n}-\frac{1}{L+\frac{1}{2}}\right], \\
& \begin{aligned}
\Delta B_{1}\left(\alpha_{\text {rel }}\right)=\frac{1}{2}(Z \alpha)^{2}[ & \alpha_{1, \text { rel }}\left\langle x^{-4}\right\rangle \\
& \left.+\left(\alpha_{2, \text { rel }}-6 \beta_{1, \text { rel }}\right)\left\langle x^{-6}\right\rangle+\cdots\right],
\end{aligned}
\end{aligned}
$$

$\Delta B_{1}\left(\phi_{1}\right)=-\frac{1}{4} \alpha^{2}\left\langle\phi_{1}\left|p^{4}\right| \phi_{0}\right\rangle_{n L}$,

and expectation values are with respect to the Rydberg electron. Equation (63) is just the Pauli approximation for the one-electron relativistic correction (in $L S$ coupling), and $\Delta B_{1}\left(\alpha_{\text {rel }}\right)$ is the energy shift due to the relativistic correction $(Z \alpha)^{2} \alpha_{\text {rel }}$ to the multipole polarizabilities [19], with $\alpha_{1}=9 /\left(2 Z^{4}\right), \quad \alpha_{1, \text { rel }}=14 /\left(3 Z^{4}\right), \quad \alpha_{2 \text {, rel }}$ $=879 /\left(40 Z^{6}\right)$, and $\beta_{1, \text { rel }}=2063 /\left(288 Z^{6}\right)$. The last is the nonadiabatic correction to $\alpha_{1 \text {, rel }}$ recently obtained by Hessels [32]. The term $\Delta B_{1}\left(\phi_{1}\right)$ defined by (65) represents the correction to the lowest-order matrix element $-\alpha^{2}\left\langle p^{4}\right\rangle_{n L} / 8$ due to the perturbing effect of the $-\alpha_{1} /\left(2 x^{4}\right)$ polarization potential on the Rydberg electron [5]. Thus $\phi_{1}$ satisfies the perturbation equation 


$$
\left(\frac{1}{2} p^{2}+V_{0}-E_{0}\right)\left|\phi_{1}\right\rangle+\left(V_{1}-E_{1}\right)\left|\phi_{0}\right\rangle=0,
$$

with

$$
\begin{aligned}
& V_{0}=-(Z-1) / x, \quad E_{0}=-(Z-1)^{2} /\left(2 n^{2}\right), \\
& V_{1}=-\alpha_{1} /\left(2 x^{4}\right), \quad E_{1}=-\alpha_{1}\left\langle x^{-4}\right\rangle / 2 .
\end{aligned}
$$

This equation can be solved analytically as a finite power-series expansion for an arbitrary $n L$ state, as discussed by Drake and Swainson [27]. Then, integrating by parts and using

$$
p^{2} \phi_{0}=2\left(E_{0}-V_{0}\right) \phi_{0}
$$

and

$$
p^{2} \phi_{1}=2\left(E_{0}-V_{0}\right) \phi_{1}+2\left(E_{1}-V_{1}\right) \phi_{0}
$$

it follows that, for $Z=2$,

$$
\begin{aligned}
\Delta B_{1}\left(\phi_{1}\right)=\alpha^{2}[ & \left\langle\phi_{1}\left|x^{-1}\left(n^{-2}-x^{-1}\right)\right| \phi_{0}\right\rangle_{n L} \\
& \left.+\frac{1}{2} \alpha_{1}\left\langle\phi_{0}\left|x^{-4}\left(n^{-2}-x^{-1}\right)\right| \phi_{0}\right\rangle_{n L}\right] .
\end{aligned}
$$

For arbitrary $Z$ and $\alpha_{1}, \Delta B_{1}\left(\phi_{1}\right)$ scales in proportion to $\alpha_{1}(Z-1)^{6}$.

A general formula for the integrals involving $\phi_{1}$ in the first term of (69) can be derived by applications of the Dalgarno interchange theorem as follows. The integral involving $x^{-1}$ can be obtained by considering an equation parallel to (66) with the perturbation being a change in the nuclear charge. If $Z \rightarrow Z+\epsilon$, then, up to first order in $\epsilon$,

$$
\left.\left\langle\phi_{1}\left|x^{-2}\right| \phi_{0}\right\rangle_{n L}=-\frac{\alpha_{1}}{2(2 L+1)} \frac{d}{d L}\left\langle x^{-4}\right\rangle_{n L}=\left\langle x^{-4}\right\rangle_{n L} \frac{\alpha_{1}}{2(2 L+1)} \mid \sum_{j=2 L-1}^{2 L+3} \frac{2}{j}+\frac{9 n-5 L(L+1) / n+2 L+1}{3 n^{2}-L(L+1)}\right) .
$$

The one additional subtlety in evaluating the above derivative with respect to $L$ arises from the fact that $n$ and $L$ are connected by the equation $n=N+L+1$, where $N$ is the fixed number of nodes, in order to preserve the boundary condition at infinity for $\phi_{0}$. Thus $n$ must change in step with $L$ such that $d n / d L=1$. Otherwise, the integral is no longer defined. The above integrals have been checked numerically. Collecting results and using

$$
\sum_{j=2 L-1}^{2 L+3} \frac{2}{j}=\frac{4(2 L-2) !}{(2 L+3) !}\left(40 f_{2}+70 f_{1}-3\right) \text {, }
$$

where $f_{1}=L(L+1)$ and $f_{2}=(L-1) L(L+1)(L+2)$, the final result is

$$
\begin{aligned}
\Delta B_{1}\left(\phi_{1}\right)=\frac{1}{2} \alpha^{2} \alpha_{1}\{ & 3\left(\frac{Z-1}{n}\right)^{2}\left\langle x^{-4}\right\rangle-(Z-1)\left\langle x^{-5}\right\rangle \\
& \left.-4 \frac{(2 L-2) !}{(2 L+3) !}\left[4\left[\frac{Z-1}{n}\right]^{6}\left[n+\frac{9 n^{2}-5 f_{1}}{2 L+1}\right]+(Z-1)^{2}\left\langle x^{-4}\right\rangle\left(\frac{40 f_{2}+70 f_{1}-3}{2 L+1}\right]\right)\right\} .
\end{aligned}
$$

Except for the additional $\Delta B_{1}\left(\phi_{1}\right)$ and $\beta_{1, \text { rel }}$ contributions, Eq. (62) corresponds to the spin-independent relativistic corrections discussed by Drachman [19]. A comparison with variational calculations is given in the following section.

Drachman [21] has obtained the leading term in the asymptotic expansion of $\left\langle B_{2}\right\rangle$ by a direct perturbation calculation, in agreement with the limit deduced from variational calculations $[3,4]$. Higher-order extensions have recently been obtained by Hessels [32] with the result 


$$
\begin{aligned}
\left\langle B_{2}\right\rangle \rightarrow \frac{\alpha^{2}}{Z^{2}}\left\{\left\langle x^{-4}\right\rangle\right. & +\frac{3(Z-1)}{Z^{2}}\left\langle x^{-5}\right\rangle \\
& -\frac{1}{Z^{2}}\left[\frac{51}{4}-\frac{27(Z-1)}{2 Z}\right. \\
& \left.\left.+\frac{3 L(L+1)}{4}\right]\left\langle x^{-6}\right\rangle\right\} .
\end{aligned}
$$

The operator $B_{2}$ scales nominally as $Z^{3}$ with nuclear charge [see Eq. (31)], but the leading term in a $Z^{-1}$ expansion of $\left\langle B_{2}\right\rangle$ vanishes exactly, resulting in the overall $(Z-1)^{4} / Z^{2} \simeq Z^{2}$ scaling of (77) due to correlation effects. Aside from the nonrelativistic energy, the differences between the left- and right-hand sides of (62) and (77) are the dominant sources of error in the asymptotic-expansion method. The differences are not fundamental, they merely represent the degree of convergence of the asymptotic expansion.

\section{Relativistic-recoil corrections}

The asymptotic expansion corresponding to the relativistic-recoil terms represented by Eq. (55) can be obtained in a fairly simple way by transforming to Jacobi coordinates. Starting from Eqs. (20) and (22), and keeping terms up to order $(\alpha y)^{2}$, the operators $H_{1}$ and $H_{4}$ in Jacobi coordinates are

$$
\begin{gathered}
H_{1}=-\frac{1}{8}(1-y)^{3} \alpha^{2}\left[\nabla_{r}^{4}-4 \Lambda y \nabla_{r} \cdot \nabla_{x} \nabla_{r}^{2}+2 \Lambda^{2} y^{2} \nabla_{r}^{2} \nabla_{x}^{2}\right. \\
\left.+4 \Lambda^{2} y^{2}\left(\nabla_{r} \cdot \nabla_{x}\right)^{2}+\Lambda^{4} \nabla_{x}^{4}\right], \\
H_{4}=\pi Z \alpha^{2}(1-y)^{2}[\delta(\mathbf{r})+\delta(\mathbf{x}+\Lambda y \mathbf{r})] .
\end{gathered}
$$

The second $\delta$ function in (79) gives a negligibly small contribution for high- $L$ states and can be neglected. The factors of $(1-y)^{3}$ and $(1-y)^{2}$ produce the reduced-mass corrections $B_{1}^{M}$ and $B_{4}^{M}$ [see Eqs. (41) and (44)], which are counted separately. What remains are the recoil terms

$$
H_{1}^{\prime}+H_{4}^{\prime}=T_{1}+T_{2}+T_{3}+T_{4}
$$

with

$$
\begin{aligned}
& T_{1}=\alpha^{2}\left[-\frac{1}{8} \nabla_{r}^{4}+\pi Z \delta(\mathbf{r})\right], \\
& T_{2}=-\frac{1}{8} \alpha^{2} \Lambda^{4} \nabla_{x}^{4}, \\
& T_{3}=\frac{1}{2} \alpha^{2} \Lambda y \nabla_{r} \cdot \nabla_{x} \nabla_{r}^{2}, \\
& T_{4}=-\frac{1}{4} \alpha^{2} y^{2} \Lambda^{2}\left[\nabla_{r}^{2} \nabla_{x}^{2}+2\left(\nabla_{r} \cdot \nabla_{x}\right)^{2}\right] .
\end{aligned}
$$

For a $1 s n L$ configuration, $T_{1}$ gives the asymptotic contributions

$$
\begin{aligned}
\left\langle T_{1}\right\rangle_{1 s n L}=\alpha^{2} Z^{2}[ & -\frac{Z^{2}}{8}+\frac{1}{2} \alpha_{1, \mathrm{rel}}\left\langle x^{-4}\right\rangle_{n L} \\
& \left.+O\left(\left\langle x^{-6}\right\rangle_{n L}\right)\right]
\end{aligned}
$$

where the first term is the relativistic energy for the $1 \mathrm{~s}$ electron, the second term is the relativistic polarizability discussed by Drachman [19], and the third term contains the relativistic quadrupole polarizability and nonadiabatic corrections. Since the transformation to Jacobi coordinates changes $\alpha_{1, \text { rel }}$ in the same way as $\alpha_{1}$ (i.e., by a factor of $C_{1}^{2}$ ), the mass-polarization correction is

$$
\left\langle T_{1}^{\mathrm{X}}\right\rangle_{1 s n L}=\alpha^{2} Z^{2} y(Z-1) \alpha_{1, \mathrm{rel}}\left\langle x^{-4}\right\rangle_{n L}
$$

$T_{2}$ gives the corresponding terms in Eq. (62) for the Rydberg electron

$$
\left\langle T_{2}\right\rangle_{1 s n L}=\Lambda^{4}\left[h_{1}(n L)+\Delta B_{1}\left(\phi_{1}\right)\right] .
$$

Expanding $\Lambda^{4} \simeq 1+4 y^{2}$, and remembering that $\phi_{1}$ changes in proportion to $\alpha_{1}$, the only significant masspolarization terms are

$$
\left\langle T_{2}^{\mathrm{X}}\right\rangle_{1 s n L}=2 y(Z-1) \Delta B_{1}\left(\phi_{1}\right)+4 y^{2} h_{1}(n L) .
$$

$T_{4}$ can also be simply evaluated, using the virial theorem to obtain

$$
\left\langle\nabla_{r}^{2} \nabla_{x}^{2}\right\rangle_{1 s n L}=Z^{2}(Z-1)^{2} / n^{2}
$$

and

$$
\left\langle\left(\nabla_{r} \cdot \nabla_{x}\right)^{2}\right\rangle_{1 s n L}=\frac{1}{3} Z^{2}(Z-1)^{2} / n^{2},
$$

with the result

$$
\left\langle T_{4}^{\mathrm{X}}\right\rangle_{1 s n L}=-\frac{5}{12} \alpha^{2} y^{2}\left(\frac{Z(Z-1)}{n}\right)^{2}
$$

$T_{3}$ is more difficult to calculate because the matrix element vanishes in a one-electron approximation, as does the adiabatic perturbation correction due to the leading dipole polarization term in Eq. (11). However, the nonadiabatic correction does not vanish. Introducing sums over intermediate states, the leading contribution is

$$
\left\langle T_{3}^{\mathrm{X}}\right\rangle_{1 s n L}=2 \sum_{n^{\prime}, n^{\prime \prime}} \frac{\left\langle 1 s n L\left|V_{1}\right| n^{\prime} p n^{\prime \prime} L \pm 1\right\rangle\left\langle n^{\prime} p n^{\prime \prime} L \pm 1\left|\left(h_{x}-e_{n}\right) T_{3}\right| 1 s n L\right\rangle}{\left(E_{1 s}-E_{n^{\prime}}\right)^{2}},
$$

where $V_{1}=r \cos (\hat{\mathbf{r}} \cdot \widehat{\mathbf{x}}) / x^{2}$ is the dipole term in Eq. (11), $E_{n^{\prime}}=-Z^{2} /\left(2 n^{\prime 2}\right)$, and $e_{n}=-(Z-1)^{2} /\left(2 n^{2}\right)$. The sums over intermediate states can be efficiently evaluated using the method of Dalgarno and Lewis [33] (see also Drachman $[18,19,21])$. To this end, we define an operator $G_{1}^{(2)}$ by

$$
V_{1}|1 s\rangle=\left[h_{r},\left[h_{r}, G_{1}^{(2)}\right]\right]|1 s\rangle .
$$

Substituting (88) into (87) gives a factor of $\left(E_{1 s}-E_{n^{\prime}}\right)^{2} G_{1}^{(2)}$ in the numerator which cancels the cor- 
responding factor in the denominator, and the sums can be completed by closure. Commuting $\left(h_{x}-e_{n}\right)$ to the right then yields

$$
\left\langle T_{3}^{\mathrm{X}}\right\rangle_{1 s n L}=2\left\langle 1 \operatorname{sn} L\left|G_{1}^{(2)}\left[h_{x}, T_{3}\right]\right| 1 \operatorname{sn} L\right\rangle .
$$

The solution to $(88)$ is

$$
G_{1}^{(2)}=\frac{\mathbf{r} \cdot \widehat{\mathbf{x}}}{Z^{4} x^{2}}\left[\frac{11}{6}+\frac{11}{12} Z r+\frac{1}{6}(Z r)^{2}\right),
$$

and the commutator in (89) is

$\left[h_{x}, T_{3}\right]|1 s n L\rangle$

$$
=\frac{1}{2} y \alpha^{2} Z^{3}(Z-1) \frac{\hat{\mathbf{r}} \cdot \hat{\mathbf{x}}}{x^{2}}\left(1-\frac{2}{Z r}-\frac{2}{Z^{2} r^{2}}\right)|1 s n L\rangle .
$$

Substituting (90) and (91) into (89) and performing the integrations gives the final result

$$
\left\langle T_{3}^{\mathrm{X}}\right\rangle_{1 \operatorname{snL} L}=-y \alpha^{2}(Z-1) \frac{20}{9}\left\langle x^{-4}\right\rangle_{n L} .
$$

The sum of $\left\langle T_{1}^{\mathrm{X}}\right\rangle$ to $\left\langle T_{4}^{\mathrm{X}}\right\rangle$ from Eqs. (83), (85), (86), and (92) yields the asymptotic matrix element

$$
\begin{aligned}
\left\langle B_{1}^{\mathrm{X}}+B_{4}^{\mathrm{X}}\right\rangle \rightarrow & y(Z \alpha)^{2}(Z-1)\left(\alpha_{1, \mathrm{rel}}-\frac{20}{9 Z^{4}}\right)\left\langle x^{-4}\right\rangle_{n L} \\
& +2 y(Z-1) \Delta B_{1}\left(\phi_{1}\right) \\
+ & y^{2}\left[-\frac{5}{12}\left[\frac{\alpha Z(Z-1)}{n}\right]^{2}+4 h_{1}(n L)\right. \\
& \left.+O\left(\alpha^{2}\left\langle x^{-4}\right\rangle_{n L}\right)\right] .
\end{aligned}
$$

It is necessary to include the terms of order $(\alpha y)^{2}$ because the leading $1 / n^{2}$ term is in fact the dominant contribution for Rydberg states down as far as $4 F$. All other terms decrease as $1 / n^{3}$.

The asymptotic limits for the spin-independent recoil terms are

$$
\left\langle B_{2}^{\mathrm{X}}\right\rangle \rightarrow-y h_{2}(n L)
$$

$$
\left\langle\Delta_{2}\right\rangle \rightarrow y\left[-\alpha^{2} Z^{4}+Z h_{2}(n L)\right],
$$

where

$$
h_{2}(n L)=\frac{\alpha^{2}(Z-1)^{3}}{n^{3}}\left(\frac{1}{n}-\frac{3}{2\left(L+\frac{1}{2}\right)}\right)
$$

is the expectation value of

$$
\frac{1}{2} \frac{\alpha^{2}}{r}\left(\nabla^{2}+\nabla_{r}^{2}\right)
$$

[see Eq. (50)]. The two terms combine to give

$$
\left\langle B_{2}^{\mathrm{X}}+\Delta_{2}\right\rangle \rightarrow-y\left[\alpha^{2} Z^{4}+(Z-1) h_{2}(n L)\right]
$$

in agreement with the recent discussion of these terms from quite a different point of view by Au, Feinberg, and Sucher [34]. Note that $\left\langle B_{2}^{X}\right\rangle$ asymptotically becomes much larger than $\left\langle B_{2}\right\rangle$ [see Eq. (94)], even though the former contains an extra factor of $y$. This is because $\left\langle B_{2}^{X}\right\rangle$ does not vanish in a one-electron approximation, while $\left\langle B_{2}\right\rangle$ does.

The asymptotic expansion for the matrix element of the $\delta$ function is known to be [35]

$$
\begin{aligned}
\pi\left\langle\delta\left(\mathbf{r}_{1}\right)\right\rangle= & Z^{3} / 2-\frac{31}{4 Z^{3}}\left\langle x^{-4}\right\rangle_{n L}+\frac{1447}{32 Z^{5}}\left\langle x^{-6}\right\rangle_{n L}+O\left(\left\langle x^{-7}\right\rangle_{n L}\right) \\
& +y\left[-\frac{31}{2 Z^{3}}(Z-1)\left\langle x^{-4}\right\rangle_{n L}+\frac{1}{16 Z^{5}}[4789+2561(Z-2)]\left\langle x^{-6}\right\rangle_{n L}\right) a_{\mu}^{-3} .
\end{aligned}
$$

This is useful in calculating QED corrections, as well as matrix elements of the Breit interaction. The polarization corrections to $\left\langle B_{2}^{\mathrm{X}}\right\rangle$ and $\left\langle\Delta_{2}\right\rangle$ of order $\left\langle x^{-4}\right\rangle_{n L}$ have not been derived, but the variational results for helium are well represented by

$$
\begin{aligned}
& \left\langle B_{2}^{X}\right\rangle \rightarrow-y\left[h_{2}(n L)-\frac{25}{16 Z^{2}}\left\langle x^{-4}\right\rangle_{n L}\right], \\
& \left\langle\Delta_{2}\right\rangle \rightarrow y\left[-\alpha^{2} Z^{4}+Z h_{2}(n L)+\frac{325}{16 Z^{2}} \alpha^{2} f(Z)\left\langle x^{-4}\right\rangle_{n L}\right] .
\end{aligned}
$$

The $Z$ scaling of the coefficient $\frac{325}{16}$ in (100) has the form

$$
f(Z)=1+b(Z-2)
$$

because of the multiplicity of terms which contribute to $\Delta_{2}$ [see Eq. (47)]. From the variational calculations, $b \simeq \frac{1}{6}$.

Adding the $B_{1}^{M}, B_{2}^{M}$, and $B_{4}^{M}$ reduced-mass terms from Eqs. (41)-(44) to the above $B_{2}^{\mathrm{X}}$ and $\Delta_{2}$ term gives 
$\left\langle-y\left(3 B_{1}+2 B_{2}+2 B_{4}\right)+B_{2}^{\mathrm{X}}+\Delta_{2}\right\rangle \rightarrow-\frac{1}{8} y \alpha^{2} Z^{4}-\frac{1}{8} y\left(\frac{(Z-1)^{2} \alpha}{n^{2}}\right)^{2}+\alpha^{2} y Z^{-2}\left\langle x^{-4}\right\rangle_{n L}\left[-7-\frac{31}{4}-2+\frac{25}{16}+\frac{325}{16} f(Z)\right]$

The first two terms are the one-electron relativistic reduced-mass shifts expected from Eq. (52), with the second term coming from the combination $y\left[-3 h_{1}(n L)+(Z-1) h_{2}(n L)\right]$. The remaining terms proportional to $\left\langle x^{-4}\right\rangle_{n L}$ come from $\alpha_{1, \text { rel }},\left\langle\delta\left(\mathbf{r}_{1}\right)\right\rangle, B_{2}$, $B_{2}^{X}$, and $\Delta_{2}$, respectively. This, together with (93), gives the total spin-independent part of the relativistic-recoil shift. For $L \geq 4$, the asymptotic expansions are at least as accurate as the variational calculations.

\section{Spin-dependent terms}

Turning now to the spin-dependent terms, the matrix elements $\left\langle B_{3}\right\rangle,\left\langle\Delta_{3}\right\rangle$, and $\left\langle B_{5}\right\rangle$ can all be simply expressed to high accuracy in terms of the single matrix element $\left\langle x^{-3}\right\rangle_{n L}[36]$ given by

$$
\left\langle x^{-3}\right\rangle_{n L}=\frac{(Z-1)^{3}}{n^{3} L(L+1 / 2)(L+1)} .
$$

Defining $T_{n L}(J)$ by

$$
\begin{aligned}
& T_{n L}(L-1)=-\alpha^{2}(L+1)\left\langle x^{-3}\right\rangle_{n L} / 4, \\
& T_{n L}(L)=-\alpha^{2}\left\langle x^{-3}\right\rangle_{n L} / 4, \\
& T_{n L}(L+1)=\alpha^{2} L\left\langle x^{-3}\right\rangle_{n L} / 4,
\end{aligned}
$$

together with

$$
S_{L}(J)=\left\{\begin{array}{l}
1 \text { for } J=L, \\
\pm 1 /(2 J+1)
\end{array} \text { for } J=L \pm 1,\right.
$$

the results for the diagonal matrix elements are

$$
\begin{aligned}
& \left\langle n L^{3} L_{J}\left|B_{3, Z}\right| n L^{3} L_{J}\right\rangle \rightarrow Z T_{n L}(J), \\
& \left\langle n L{ }^{3} L_{J}\left|B_{3, e}\right| n L^{3} L_{J}\right\rangle \rightarrow-3 T_{n L}(J), \\
& \left\langle n L^{3} L_{J}\left|B_{5}\right| n L{ }^{3} L_{J}\right\rangle \rightarrow 2 S_{L}(J) T_{n L}(J), \\
& \left\langle n L^{3} L_{J}\left|B_{3, Z}^{\mathrm{X}}\right| n L^{3} L_{J}\right\rangle \rightarrow y T_{n L}(J), \\
& \left\langle n L^{3} L_{J}\left|B_{3, e}^{\mathrm{X}}\right| n L^{3} L_{J}\right\rangle \rightarrow-3 y T_{n L}(J), \\
& \left\langle n L^{3} L_{J}\left|\Delta_{3}\right| n L^{3} L_{J}\right\rangle \rightarrow-2 y T_{n L}(J), \\
& \left\langle n L^{3} L_{J}\left|B_{5}^{X}\right| n L^{3} L_{J}\right\rangle \rightarrow 0+O\left[(\alpha y)^{2}\right] .
\end{aligned}
$$

The off-diagonal matrix elements are

$$
\begin{aligned}
& \left\langle n L^{3} L_{L}\left|B_{3, Z}\right| n L^{1} L_{L}\right\rangle \rightarrow Z T_{n L}(L)[L(L+1)]^{1 / 2}, \\
& \left\langle n L^{3} L_{L}\left|B_{3, e}\right| n L{ }^{1} L_{L}\right\rangle \rightarrow T_{n L}(L)[L(L+1)]^{1 / 2}, \\
& \left\langle n L^{3} L_{L}\left|B_{3, Z}^{\mathrm{X}}\right| n L{ }^{1} L_{L}\right\rangle \rightarrow-y T_{n L}(L)[L(L+1)]^{1 / 2}, \\
& \left\langle n L^{3} L_{L}\left|B_{3, e}^{\mathrm{X}}\right| n L{ }^{1} L_{L}\right\rangle \rightarrow-y T_{n L}(L)[L(L+1)]^{1 / 2}, \\
& \left\langle n L^{3} L_{L}\left|\Delta_{3}\right| n L{ }^{1} L_{L}\right\rangle \rightarrow 2 y T_{n L}(L)[L(L+1)]^{1 / 2} .
\end{aligned}
$$

The complete matrix elements, including the reducedmass and anomalous-magnetic-moment corrections from Eqs. (49) and (61), are thus

$$
\begin{aligned}
\left\langle n L^{3} L_{J}\right| B_{3}+B_{5}+B_{3}^{\mathrm{X}}+B_{5}^{\mathrm{X}}+B_{3}^{M} & +B_{5}^{M}+\Delta_{3}+B_{3}^{A}+B_{5}^{A}\left|n L{ }^{3} L_{J}\right\rangle \\
& \rightarrow T_{n L}(J)\left[Z-3+2 S_{L}(J)+\frac{\mu}{M}\left[2-4 S_{L}(J)\right]+2 \gamma_{e}\left[Z-2+\left(2+\gamma_{e}\right) S_{L}(J)\right]\right)
\end{aligned}
$$

and

$$
\begin{aligned}
\left\langle n L^{3} L_{L}\right| & B_{3}+B_{3}^{\mathrm{X}}+B_{3}^{M}+\Delta_{3}+B_{3}^{A}\left|n L^{1} L_{L}\right\rangle \\
& \rightarrow T_{n L}(L)\left(Z+1-2 \frac{\mu}{M}+2 \gamma_{e} Z\right)[L(L+1)]^{1 / 2}
\end{aligned}
$$

in units of $e^{2} / a_{\mu}$, with $\gamma_{e} \simeq \alpha / 2 \pi-0.32848(\alpha / \pi)^{2}$. It is interesting that the $Z$ dependence of the relativistic-recoil plus reduced-mass terms cancels in the asymptotic limit.

The above matrix elements of $B_{3, Z}, B_{3, e}$, and $B_{5}$ follow in a simple way from the asymptotic forms of the operators themselves. Concerning $\Delta_{3}$, its matrix elements seem surprising at first sight because the expectation values of $\mathbf{r}_{1} \times \mathbf{p}_{2}$ and $\mathbf{r}_{2} \times \mathbf{p}_{1}$ [see Eq. (48)] vanish in any one- electron approximation. However, nonvanishing contributions proportional to $T_{n L}(J)$ come from first-order polarization corrections to the wave functions, as can be shown by a direct perturbation calculation (see the Appendix). Since the matrix elements vanish in lowest order, the $Z$ scaling of $\left\langle\Delta_{3}\right\rangle$ is one power of $Z$ lower than the nominal $Z^{4}$ scaling indicated by Eq. (48). Furthermore, a transformation to Jacobi coordinates shows that in the asymptotic limit, $\Delta_{3} \rightarrow-2 B_{3, Z}^{\mathrm{X}}$ (see the Appendix). This establishes the correct $Z$ scaling of $B_{3, Z}^{\mathrm{X}}$ and ties together the relative signs of the off-diagonal matrix elements. A comparison with the derivation of Au, Feinberg, and Sucher [34] is not meaningful for this case because their effective two-body formalism does not contain a complete representation of the spin-dependent interactions. The derivation of Hessels et al. [36] corresponds to replacing $\Delta_{3}$ and $B_{3}^{M}$ by $\widetilde{\Delta}_{3}=\Delta_{3}+2 y B_{3, Z}$ and 
$\widetilde{B}_{3}^{M}=B_{3}^{M}-2 y B_{3, Z}$, which restores the terms that were canceled in deriving $\Delta_{3}$ [see the discussion following Eq. (48)], and then neglecting the contributions from $\widetilde{\Delta}_{3}$, $B_{3, Z}^{\mathrm{X}}$, and $B_{3, e}^{\mathrm{X}}$. The neglected terms sum to zero (asymp- totically) for the off-diagonal matrix element.

For completeness, the finite-mass corrections to the anomalous-magnetic-moment terms can be extracted directly from Eqs. (25) - (27). They are

$$
\begin{aligned}
-2 \gamma_{e} y\left\langle B_{3, Z}+\frac{4}{3} \delta_{S, S^{\prime}} B_{3, e}+2 B_{5}\right\rangle+\gamma_{e}\left\langle 2 B_{3, Z}^{\mathrm{X}}+\frac{4}{3} \delta_{S, S^{\prime}} B_{3, e}^{\mathrm{X}}+\Delta_{3}+2 B_{5}^{\mathrm{X}}\right\rangle \\
\rightarrow\left\{\begin{array}{l}
-2 \gamma_{e} y\left[Z-2+4 S_{L}(J)\right] T_{n L}(J) \quad \text { for } S=S^{\prime}=1, \\
-2 \gamma_{e} y Z T_{n L}(L)[L(L+1)]^{1 / 2} \text { for } S=1, \quad S^{\prime}=0 .
\end{array}\right.
\end{aligned}
$$

The only term not included so far in the asymptotic expansions is the term proportional to $\pi\left\langle\delta\left(\mathbf{r}_{12}\right)\right\rangle$ in Eq. (39). In a simple screening approximation, with $R_{n L}(r, Z)$ the hydrogenic radial wave function for nuclear charge $Z$, the matrix element is given by

$$
\begin{aligned}
\pi\left\langle\delta\left(\mathbf{r}_{12}\right)\right\rangle & =\frac{1}{2} \int_{0}^{\infty}\left|R_{1 s}(r, Z)\right|^{2}\left|R_{n L}(r, Z-1)\right|^{2} r^{2} d r \\
& =\frac{2 Z^{3}(n+L) !}{(2 L+1) !(n-L-1) !}\left(\frac{Z-1}{n Z}\right)^{2 L+4}\left(\frac{Z L+1}{Z-1}\right) e^{-2(Z-1) / Z}
\end{aligned}
$$

and so decreases exponentially with $L$. However, the above is asymptotically larger than the actual variational matrix elements (see Sec. IV) by approximately a factor of 4 for helium. As a function of $Z$, the required asymptotic correction factor is approximately

$$
g(Z)=\left(\frac{Z-1}{Z}\right)^{2}
$$

With $g(Z)$ included, the above reproduces the variational calculations to within $18 \%$ for $L \geq 4$. For the low- $L$ states of helium, the correction factors are $2.07 \mathrm{~g}(Z)$, $1.58 g(Z), 1.32 g(Z), 1.18 g(Z)$, and $1.08 g(Z)$, respectively, for $L=1,2,3,4$, and 5 , with little dependence on $n$. That $g(Z)$ is substantially smaller than unity indicates that correlation effects and the "Coulomb hole" [37] about the point $\mathbf{r}_{12}=0$ continue to play an important role, even in the asymptotic limit.

\section{COMPARISON WITH VARIATIONAL CALCULATIONS}

A comparison of the asymptotic expansions with matrix elements obtained from high-precision variational wave functions serves two purposes. First, for low to moderate $L$, it allows a precise assessment of the accuracy of the truncated asymptotic expansions. Second, for high $L$, the comparison should be regarded instead as a test of the variational results. Since the rate of convergence of the asymptotic expansions rapidly improves with increasing $L$, the expansions eventually exceed the accuracy of the variational matrix elements.

\section{A. Nonrelativistic energies}

Tables I and II summarize the nonrelativistic energies for infinite nuclear mass, together with the first- and second-order mass-polarization corrections. This and the subsequent tables include the $2 S$ states and all higher- $L$ states up to $n=10$ and $L=7$. As an example of the spectroscopic notation, $2 P$ means $1 s 2 p^{1} P$ or ${ }^{3} P$. A full dis- cussion of the double basis-set variational methods and convergence studies for each state can be found in Ref. [5], together with comparisons with previous work. Detailed comparisons with the asymptotic expansions for the nonrelativistic energies have been presented previously $[4,21]$ and will not be repeated here. However, comparisons with the asymptotic expansions for the first- and second-order mass-polarization corrections (in units of $\left.e^{2} / a_{\mu}\right)$

$$
\varepsilon_{M}^{(1)}=\left\langle\mathbf{p}_{1} \cdot \mathbf{p}_{2}\right\rangle
$$

and

$$
\varepsilon_{M}^{(2)} \simeq\left[E_{M}-E_{\infty}-y \varepsilon_{M}^{(1)}\right] / y^{2}
$$

to the nonrelativistic energies provide important tests of the variational results. In Eq. (114), $E_{\infty}$ is the energy for infinite nuclear mass, and $E_{M}$ is the energy corresponding to ${ }^{4} \mathrm{He}$ obtained by explicitly including the $y \mathbf{p}_{1} \cdot \mathbf{p}_{2}$ mass-polarization term in the Hamiltonian $\left(y=1.370745620 \times 10^{-4}\right)$. The $\varepsilon_{M}^{(2)}$ comparison is particularly interesting because it provides a profound test of the variational calculations to the full extent of their estimated convergence. The extreme sensitivity follows from the fact that the terms $E_{\infty}+y \varepsilon_{M}^{(1)}$ in Eq. (114) account for the first nine significant figures of $E_{M}$, and so the first significant figure of $\varepsilon_{M}^{(2)}$ is determined by the tenth significant figure of $E_{M}$. A failure of the variational basis sets to converge to the correct answer (relative to $\left.E_{\infty}\right)$ is immediately revealed by a comparison with the asymptotic $\varepsilon_{M}^{(2)}$ from Eq. (18). The one slight complication is that the variational estimate of $\varepsilon_{M}^{(2)}$ from Eq. (114) is contaminated by terms of higher order in $y$. For the present levels of accuracy, the only significant such term is the contribution $-y^{4}(Z-1)^{2} /\left(2 n^{2}\right)$ in Eq. (10) to $y^{2} \varepsilon_{M}^{(2)}$. The asymptotic $\varepsilon_{M}^{(2)}$ is therefore modified to be

$$
\widetilde{\varepsilon}_{M}^{(2)}(y)=\varepsilon_{M}^{(2)}-y^{2}(Z-1)^{2} /\left(2 n^{2}\right)
$$

in order to compare with the variational values from Eq. 
(114) for ${ }^{4} \mathrm{He}$. The small-y dependence of $\varepsilon_{M}^{(2)}$ should be taken into account for very-high-precision applications to isotope shifts, etc.

Table III shows the comparison for $\varepsilon_{M}^{(1)}$. The asymptotic expansion for the $H, I$, and $K$ states includes the new Drachman [21] terms of order $\left\langle x^{-9}\right\rangle_{n L}$ and $\left\langle x^{-10}\right\rangle_{n L}$ shown in Eq. (14). As recommended by him, the quantity added is $\frac{1}{4}\left(c_{9}^{(1)}\left\langle x^{-9}\right\rangle_{n L}+c_{10}^{(1)}\left\langle x^{-10}\right\rangle_{n L}\right)$ with $\pm \frac{1}{4}\left(c_{9}^{(1)}\left\langle x^{-9}\right\rangle_{n L}+c_{10}^{(1)}\left\langle x^{-10}\right\rangle_{n L}\right)$ regarded as the uncertainty. These terms improve the agreement with the variational results by about one significant figure. In every case, the actual differences are close to the uncertainty estimate for the asymptotic expansion.

As discussed above, the comparison for $\widetilde{\varepsilon}_{M}^{(2)}(y)$ in Table IV provides instead a test of the variational results. For $L>3$, the asymptotic expansion (15) [including Eq. (115)] becomes the more accurate of the two. The differences are in reasonably good accord with the accuracies estimated from the apparent convergence of the variational calculations [5]. At present levels of accuracy, terms beyond $\left\langle x^{-8}\right\rangle_{n L}$ in the asymptotic expansion are not necessary, although they are known [26] and are included

TABLE I. Nonrelativistic variational energies $E_{\infty}=-2-1 /\left(2 n^{2}\right)+\Delta E_{\infty}$ for infinite nuclear mass, and first- and second-order mass-polarization coefficients $\varepsilon_{M}^{(1)}$ and $\varepsilon_{M}^{(2)}$ for the singlet states of helium (in units of $10^{-3}$ a.u.).

\begin{tabular}{|c|c|c|c|}
\hline State & $\Delta E_{\infty}\left(n^{1} L\right)$ & $\varepsilon_{M}^{(1)}\left(n^{1} L\right)$ & $\varepsilon_{M}^{(2)}\left(n^{1} L\right)$ \\
\hline $2 S$ & $-20.97404605443(5)$ & $9.5038644190(2)$ & $-135.27689(2)$ \\
\hline $\begin{array}{r}2 P \\
3 P \\
4 P \\
5 P \\
6 P \\
7 P \\
8 P \\
9 P \\
10 P\end{array}$ & $\begin{array}{l}1.156913501908(8) \\
0.40919346361(3) \\
0.18034954976(3) \\
0.09401009917(2) \\
0.05490921715(2) \\
0.03476710330(2) \\
0.02337286678(2) \\
0.01645485331(5) \\
0.01201619778(4)\end{array}$ & $\begin{array}{c}46.04452494(1) \\
14.548047097(1) \\
6.2549235543(1) \\
3.23002184(2) \\
1.878058536(1) \\
1.18615230(1) \\
0.79619583(5) \\
0.559978028(2) \\
0.408649426(2)\end{array}$ & $\begin{array}{c}-168.27140(5) \\
-66.047859(3) \\
-35.15971(6) \\
-21.8476(3) \\
-14.90286(9) \\
-10.8186(2) \\
-8.2117(5) \\
-6.4457(2) \\
-5.197(1)\end{array}$ \\
\hline $\begin{array}{r}3 D \\
4 D \\
5 D \\
6 D \\
7 D \\
8 D \\
9 D \\
10 D\end{array}$ & $\begin{array}{l}-0.065177296690(6) \\
-0.029846178687(7) \\
-0.015836159984(4) \\
-0.009338535397(5) \\
-0.00594682532(1) \\
-0.004012563811(7) \\
-0.002831931468(6) \\
-0.002071654250(6)\end{array}$ & $\begin{array}{l}-0.2493999921(1) \\
-0.1291751887(8) \\
-0.071883131(6) \\
-0.0434122689(9) \\
-0.028027840(2) \\
-0.019076181(1) \\
-0.013542185(3) \\
-0.0099475060(6)\end{array}$ & $\begin{array}{l}-57.201299(9) \\
-32.15091(2) \\
-20.5101(2) \\
-14.1994(2) \\
-10.40509(3) \\
-7.9507(4) \\
-6.27099(7) \\
-5.0724(4)\end{array}$ \\
\hline $\begin{array}{r}4 F \\
5 F \\
6 F \\
7 F \\
8 F \\
9 F \\
10 F\end{array}$ & $\begin{array}{l}-0.005144381749(1) \\
-0.0029371587427(5) \\
-0.0017949266608(3) \\
-0.0011664413586(3) \\
-0.0007971150141(6) \\
-0.0005673911518(8) \\
-0.000417564669(2)\end{array}$ & $\begin{array}{l}-0.0100242694(2) \\
-0.0057042946(4) \\
-0.003482257(7) \\
-0.00226200(4) \\
-0.00154548(1) \\
-0.0010999671(3) \\
-0.000809442(9)\end{array}$ & $\begin{array}{c}-31.274336(4) \\
-20.013498(6) \\
-13.896984(2) \\
-10.2092(3) \\
-7.8159(3) \\
-6.17520(1) \\
-5.00176(2)\end{array}$ \\
\hline $\begin{array}{r}5 G \\
6 G \\
7 G \\
8 G \\
9 G \\
10 G\end{array}$ & $\begin{array}{l}-0.000710898584714(7) \\
-0.00045649842434(3) \\
-0.00030459211949(7) \\
-0.0002114940241(1) \\
-0.0001521214135(1) \\
-0.0001127643182(4)\end{array}$ & $\begin{array}{l}-0.00140441364(4) \\
-0.0008985799(7) \\
-0.0005983963(3) \\
-0.00041500403(5) \\
-0.0002982672(1) \\
-0.000220982(2)\end{array}$ & $\begin{array}{l}-20.00356085(5) \\
-13.891179(6) \\
-10.20561(5) \\
-7.813563(1) \\
-6.1735796(1) \\
-5.00055(2)\end{array}$ \\
\hline $\begin{array}{r}6 H \\
7 H \\
8 H \\
9 H \\
10 H\end{array}$ & $\begin{array}{l}-0.0001458653908318(7) \\
-0.00010117382898(2) \\
-0.00007182865581(1) \\
-0.00005239744630(2) \\
-0.00003921439452(2)\end{array}$ & $\begin{array}{l}-0.0002903470899(3) \\
-0.00020109779(3) \\
-0.0001426492(3) \\
-0.000104002216(2) \\
-0.00007780668(4)\end{array}$ & $\begin{array}{l}-13.88961906(3) \\
-10.204590(2) \\
-7.812855(4) \\
-6.173104(2) \\
-5.0001935(2)\end{array}$ \\
\hline $\begin{array}{r}7 I \\
8 I \\
9 I \\
10 I\end{array}$ & $\begin{array}{l}-0.0000389735382601(1) \\
-0.0000285495845853(4) \\
-0.000021226209733(1) \\
-0.000016086516194(2)\end{array}$ & $\begin{array}{l}-0.000077775526(3) \\
-0.00005693591(1) \\
-0.00004231359(3) \\
-0.0000320590(1)\end{array}$ & $\begin{array}{c}-10.20427673(2) \\
-7.81264288(1) \\
-6.1729459(1) \\
-5.0000803(4)\end{array}$ \\
\hline $\begin{array}{r}8 K \\
9 K \\
10 K\end{array}$ & $\begin{array}{l}-0.0000125702293050(0) \\
-0.0000095901569404(1) \\
-0.0000073883758771(7)\end{array}$ & $\begin{array}{l}-0.0000251113316(0) \\
-0.0000191516195(1) \\
-0.000014751409(2)\end{array}$ & $\begin{array}{l}-7.81256302(1) \\
-6.17288759(1) \\
-5.00003693(5)\end{array}$ \\
\hline
\end{tabular}


in the tabulated values. Even for the $P$ states, where only the leading two terms of Eq. (15) are nondivergent, deviations from a systematic trend in the differences can indicate errors in the variational eigenvalues as small as $10^{-13}$ a.u. For example, for the $10 P$ state in Table IV, a change of $0.01 \times 10^{-3}$ in the "difference" of $0.078(1) \times 10^{-3}$ would correspond to an energy change of $0.01 \times 10^{-3} y^{2}=1.88 \times 10^{-13}$ a.u. The eventual dominance of $y^{2} \varepsilon_{M}^{(2)}$ over $y \varepsilon_{M}^{(1)}$ for sufficiently high $L$ is evident from the table.

\section{B. Relativistic corrections}

Aside from the nonrelativistic energy, the largest source of error in the asymptotic-expansion method is the lowest-order relativistic correction. Beginning with the spin-independent part given by Eq. (62), the variational matrix elements $-\frac{1}{8}\left\langle p_{1}^{4}+p_{2}^{4}\right\rangle$ are listed for the singlet and triplet states in Table $V$. The matrix elements $\pi\left\langle\delta\left(\mathbf{r}_{1}\right)\right\rangle$ are given separately in Table VI for $L$ up to 2 . This completes the tabulation of these matrix elements given previously for $3 \leq L \leq 7$ [35]. The left-hand side of Eq. (62) also contains the term $\alpha^{2}\left\langle\pi \delta\left(\mathbf{r}_{12}\right)\right\rangle$, but as shown in Eq. (111) (see also Table VII), this decreases exponentially with $L$, and is negligible for $L>3$.

The comparison in Table VIII with the asymptotic expansion shows that the $\Delta B_{1}\left(\phi_{1}\right)$ term removes what would otherwise be a significant discrepancy with the

TABLE II. Same as Table I for the triplet states of helium (in units of $10^{-3}$ a.u.).

\begin{tabular}{|c|c|c|c|}
\hline State & $\Delta E_{\infty}\left(n^{3} L\right)$ & $\varepsilon_{M}^{(1)}\left(n^{3} L\right)$ & $\varepsilon_{M}^{(2)}\left(n^{3} L\right)$ \\
\hline $2 S$ & $-50.2293782367912(1)$ & $7.442130705(1)$ & $-57.495840(8)$ \\
\hline $\begin{array}{r}2 P \\
3 P \\
4 P \\
5 P \\
6 P \\
7 P \\
8 P \\
9 P \\
10 P\end{array}$ & $\begin{array}{l}-8.16419077927(1) \\
-2.52552871872(4) \\
-1.07435429662(2) \\
-0.55118725625(1) \\
-0.31906988485(1) \\
-0.20087837528(2) \\
-0.13451377112(1) \\
-0.09442786024(4) \\
-0.0688054978(1)\end{array}$ & $\begin{array}{l}-64.572425024(3) \\
-18.369001636(2) \\
-7.55517898(1) \\
-3.810911035(1) \\
-2.184346463(1) \\
-1.3665008(3) \\
-0.9110535(3) \\
-0.637531359(5) \\
-0.463433718(8)\end{array}$ & $\begin{array}{c}-204.95993(2) \\
-70.292710(2) \\
-36.129973(2) \\
-22.16661(9) \\
-15.03358(5) \\
-10.879(2) \\
-8.2487(6) \\
-6.464937(1) \\
-5.2067(1)\end{array}$ \\
\hline $\begin{array}{r}3 D \\
4 D \\
5 D \\
6 D \\
7 D \\
8 D \\
9 D \\
10 D\end{array}$ & $\begin{array}{l}-0.080753897706(4) \\
-0.038847501795(3) \\
-0.021027446911(5) \\
-0.012526564903(7) \\
-0.008024322942(2) \\
-0.005434711706(3) \\
-0.003845378524(2) \\
-0.002818080232(8)\end{array}$ & $\begin{array}{l}0.025322839(1) \\
0.029442651(2) \\
0.01956885(1) \\
0.01274222(3) \\
0.008563121(3) \\
0.0059711234(3) \\
0.004306538(6) \\
0.003198298(8)\end{array}$ & $\begin{array}{c}-54.73773(1) \\
-30.747891(7) \\
-19.7062(2) \\
-13.70727(1) \\
-10.085212(1) \\
-7.73159(2) \\
-6.1152(1) \\
-4.9580(8)\end{array}$ \\
\hline $\begin{array}{r}4 F \\
5 F \\
6 F \\
7 F \\
8 F \\
9 F \\
10 F\end{array}$ & $\begin{array}{l}-0.0051684032456(6) \\
-0.0029573773694(4) \\
-0.0018094596431(2) \\
-0.0011767422112(2) \\
-0.0008045350908(5) \\
-0.0005728588702(7) \\
-0.000421686604(1)\end{array}$ & $\begin{array}{l}-0.009669639550(9) \\
-0.0054064900(5) \\
-0.0032684586(8) \\
-0.00211058(3) \\
-0.001436452(2) \\
-0.001019651(2) \\
-0.0007489264(2)\end{array}$ & $\begin{array}{l}-31.2779921(3) \\
-20.016561(4) \\
-13.89922(3) \\
-10.2107(3) \\
-7.8170(2) \\
-6.1760254(7) \\
-5.002386(2)\end{array}$ \\
\hline $\begin{array}{r}5 G \\
6 G \\
7 G \\
8 G \\
9 G \\
10 G\end{array}$ & $\begin{array}{l}-0.000710925343925(4) \\
-0.00045652806407(3) \\
-0.00030461756487(6) \\
-0.00021151442482(9) \\
-0.0001521374922(3) \\
-0.0001127770033(6)\end{array}$ & $\begin{array}{l}-0.00140400125(2) \\
-0.0008981237(7) \\
-0.000598005(1) \\
-0.00041469037(1) \\
-0.00029801982(6) \\
-0.000220785(3)\end{array}$ & $\begin{array}{l}-20.00356458(5) \\
-13.891184(8) \\
-10.20561(5) \\
-7.813568(2) \\
-6.173592(4) \\
-5.00055(2)\end{array}$ \\
\hline $\begin{array}{r}6 H \\
7 H \\
8 H \\
9 H \\
10 H\end{array}$ & $\begin{array}{l}-0.0001458654126648(6) \\
-0.000101173858985(8) \\
-0.00007182868573(1) \\
-0.00005239747304(2) \\
-0.00003921441741(2)\end{array}$ & $\begin{array}{l}-0.0002903467263(1) \\
-0.000201097257(7) \\
-0.0001426487(2) \\
-0.00010400189(2) \\
-0.00007780622(1)\end{array}$ & $\begin{array}{l}-13.88961897(3) \\
-10.204587(2) \\
-7.812859(5) \\
-6.173101(2) \\
-5.00019346(7)\end{array}$ \\
\hline $\begin{array}{r}7 I \\
8 I \\
9 I \\
10 I\end{array}$ & $\begin{array}{l}-0.0000389735382737(1) \\
-0.000028549584608(1) \\
-0.0000212262097577(6) \\
-0.000016086516219(2)\end{array}$ & $\begin{array}{l}-0.000077775520(3) \\
-0.00005693591(2) \\
-0.00004231362(5) \\
-0.0000320589(2)\end{array}$ & $\begin{array}{l}-10.20427678(1) \\
-7.81264294(4) \\
-6.1729460(2) \\
-5.000081(1)\end{array}$ \\
\hline $\begin{array}{r}8 K \\
9 K \\
10 K\end{array}$ & $\begin{array}{l}-0.0000125702293050(0) \\
-0.0000095901569404(1) \\
-0.0000073883758771(7)\end{array}$ & $\begin{array}{l}-0.0000251113317(0) \\
-0.0000191516197(3) \\
-0.000014751413(6)\end{array}$ & $\begin{array}{l}-7.81256302(1) \\
-6.17288759(1) \\
-5.00003683(2)\end{array}$ \\
\hline
\end{tabular}


variational matrix elements. The residual differences decrease more rapidly with $L$ than $\left\langle x^{-6}\right\rangle_{n L}$ since the relativistic quadrupole polarizability [19] and nonadiabatic correction terms [32] are included. The close agreement leaves little room for doubt that the variational results are correct.

The above does not include the contribution from $\left\langle B_{2}\right\rangle$ because this corresponds asymptotically to the largest part of what is called the retardation term in the asymptotic expansion and LRI pictures [see Eq. (120) below]. Table IX lists the variational matrix elements, and Table $\mathrm{X}$ shows the good agreement that is obtained with the asymptotic expansion (77) for high $L$, provided that the higher-order corrections in (77) are included.

Turning now to the relativistic-recoil terms, the asymptotic expansion Eq. (93) does not quite correspond to the variational matrix elements because of terms of order $\alpha^{2} y^{2}$. Although the mass-polarization operator is included to all orders in the variational matrix elements, the term $-y^{2}\left(\mathbf{p}_{1} \cdot \mathbf{p}_{2}\right)^{2} / 2$, which was dropped from $\mathrm{Eq}$. (28), contributes to the $1 / n^{2}$ term. Its expectation value is asymptotically $-(\alpha y)^{2}\left(\mathbf{p}_{1} \cdot \mathbf{p}_{2}\right)^{2} / 2 \rightarrow-(\alpha y)^{2}[Z(Z-1) / n]^{2} / 6$,

and this is precisely the difference between Eq. (93) and the high- $L$ limit of the variational results. If this term is of importance $\left(-4.39 / n^{2} \mathrm{kHz}\right.$ for $\left.{ }^{4} \mathrm{He}\right)$, then it should be added to $\left(\Delta E_{\mathrm{RR}}\right)_{\mathrm{X}}$ [see Eqs. (54) and (55)] and the total energy obtained from the variational matrix elements. Table XI compares the two methods of calculation, with the missing term in Eq. (116) subtracted from the asymptotic expansion. The variational results correspond to the quantity (in a.u.)

$$
\left\langle B_{1}^{X}+B_{4}^{X}\right\rangle=\alpha^{2}(\mu / M)\left(p_{4}^{X}+Z d_{1}^{X}\right)
$$

with $p_{4}^{X}$ from Table $\mathbf{V}$ and $d_{1}^{X}$ from Table VI (or Table IV of Ref. [35] for $L>2$ ). For consistency with the asymptotic expansions, the small $-d_{12}^{X}$ term [see Eq. (32)] listed in Table VII is not included. For $L \geq 4$, the asymptotic expansion becomes comparable in accuracy to the variational results owing to loss of precision in the latter due to the differencing of nearly equal numbers [see Eq. (114)]. In addition, there is severe cancellation between the singlet and triplet $D$ states on taking the spin average.

TABLE III. Comparison of spin-averaged variational matrix elements with the asymptotic values [see Eq. (14)] for the first-order mass-polarization coefficient $\varepsilon_{M}^{(1)}$ (in units of $10^{-3}$ a.u.).

\begin{tabular}{|c|c|c|c|}
\hline State & Variational & Asymptotic & Difference \\
\hline $\begin{array}{r}3 D \\
4 D \\
5 D \\
6 D \\
7 D \\
8 D \\
9 D \\
10 D\end{array}$ & $\begin{array}{l}-0.112038577(1) \\
-0.049866269(2) \\
-0.02615714(2) \\
-0.01533502(3) \\
-0.009732359(4) \\
-0.006552529(1) \\
-0.004617823(6) \\
-0.003374604(8)\end{array}$ & $\begin{array}{l}-0.10(6) \\
-0.04(3) \\
-0.02(2) \\
-0.01(1) \\
-0.008(8) \\
-0.005(5) \\
-0.004(4) \\
-0.003(3)\end{array}$ & $\begin{array}{l}-0.01(6) \\
-0.01(3) \\
-0.00(2) \\
-0.00(1) \\
-0.002(8) \\
-0.001(5) \\
-0.001(4) \\
-0.001(3)\end{array}$ \\
\hline $\begin{array}{r}4 F \\
5 F \\
6 F \\
7 F \\
8 F \\
9 F \\
10 F\end{array}$ & $\begin{array}{l}-0.0098469545(2) \\
-0.0055553923(6) \\
-0.003375358(7) \\
-0.00218629(5) \\
-0.00149097(1) \\
-0.001059809(2) \\
-0.000779184(9)\end{array}$ & $\begin{array}{l}-0.0101(4) \\
-0.0057(3) \\
-0.0035(2) \\
-0.0023(1) \\
-0.00155(9) \\
-0.00110(7) \\
-0.00081(5)\end{array}$ & $\begin{array}{l}0.0003(4) \\
0.0002(3) \\
0.0001(2) \\
0.0001(1) \\
0.00006(9) \\
0.00004(7) \\
0.00003(5)\end{array}$ \\
\hline $\begin{array}{r}5 G \\
6 G \\
7 G \\
8 G \\
9 G \\
10 G\end{array}$ & $\begin{array}{l}-0.00140420744(5) \\
-0.000898352(1) \\
-0.000598201(1) \\
-0.00041484720(5) \\
-0.0002981435(1) \\
-0.000220883(3)\end{array}$ & $\begin{array}{l}-0.0014037(9) \\
-0.000898(1) \\
-0.0005978(8) \\
-0.0004146(6) \\
-0.0002979(5) \\
-0.0002207(4)\end{array}$ & $\begin{array}{l}-0.0000005(9) \\
-0.000000(1) \\
-0.0000004(8) \\
-0.0000002(6) \\
-0.0000002(5) \\
-0.0000002(4)\end{array}$ \\
\hline $\begin{array}{r}6 H \\
7 H \\
8 H \\
9 H \\
10 H\end{array}$ & $\begin{array}{l}-0.0002903469081(3) \\
-0.00020109752(3) \\
-0.0001426489(4) \\
-0.00010400205(2) \\
-0.00007780645(4)\end{array}$ & $\begin{array}{l}-0.000290348(3) \\
-0.000201098(3) \\
-0.000142650(3) \\
-0.000104003(3) \\
-0.000077807(2)\end{array}$ & $\begin{array}{l}0.000000001(2) \\
0.000000001(3) \\
0.000000001(3) \\
0.000000001(3) \\
0.000000001(2)\end{array}$ \\
\hline $\begin{array}{r}7 I \\
8 I \\
9 I \\
10 I\end{array}$ & $\begin{array}{l}-0.000077775523(4) \\
-0.00005693591(2) \\
-0.00004231360(6) \\
-0.0000320589(3)\end{array}$ & $\begin{array}{l}-0.00007777554(3) \\
-0.00005693594(5) \\
-0.00004231367(5) \\
-0.00003205900(5)\end{array}$ & $\begin{array}{l}0.00000000002(3) \\
0.00000000003(5) \\
0.00000000006(8) \\
0.0000000000(3)\end{array}$ \\
\hline $\begin{array}{r}8 K \\
9 K \\
10 K\end{array}$ & $\begin{array}{l}-0.000025111331651(1) \\
-0.0000191516196(3) \\
-0.000014751411(7)\end{array}$ & $\begin{array}{l}-0.000025111332(1) \\
-0.000019151621(2) \\
-0.000014751390(2)\end{array}$ & $\begin{array}{r}0.000000000001(1) \\
0.000000000002(2) \\
-0.000000000021(7)\end{array}$ \\
\hline
\end{tabular}


For the $F$ states, there is severe cancellation between the positive terms of order $\alpha^{2} y$ and the negative terms of order $\alpha^{2} y^{2}$ in Eq. (93). For higher $L$, the latter terms become dominant, which explains the changes in sign evident in Table XI and $p_{4}^{X}$ in Table $V$. The residual differences in the last column are approximately $-4 \alpha^{2} y\left\langle x^{-6}\right\rangle_{n L}$ a.u., which is taken to be the uncertainty in the asymptotic values. Matrix elements of the remaining recoil term $\Delta_{2}$ are listed in Table XII. The asymptotic form is given by Eq. (100).

For completeness, Table XIII lists the variational values for the $Q$ matrix elements defined by

$$
Q=\frac{1}{4 \pi} \lim _{a \rightarrow 0}\left\langle r_{12}^{-3}(a)+4 \pi(\gamma+\ln a) \delta\left(\mathbf{r}_{12}\right)\right\rangle,
$$

where $\gamma$ is Euler's constant and $a$ is the radius of a sphere centered at $r_{12}=0$ which is excluded from the integration over $r_{12}$. This is required in the calculation of the ArakiSucher electron-electron QED contribution to the energy given by $[38,39]$

$\Delta E_{L, 2}(n L S)=\alpha^{3}\left(\frac{14}{3} \ln \alpha+\frac{164}{15}\right)\left\langle\delta\left(\mathbf{r}_{12}\right)\right\rangle-\frac{14}{3} \alpha^{3} Q$.

The above contains contributions from one- and twophoton exchange, vertex terms, vacuum-polarization terms, anomalous-magnetic-moment terms, and Coulomb corrections. For Rydberg states $\left\langle\delta\left(\mathbf{r}_{12}\right)\right\rangle$ decreases exponentially with $L$ and can be neglected (see Table VII). The $Q$ term is well approximated by its asymptotic expansion

TABLE IV. Comparison of spin-averaged variational matrix elements with the asymptotic values [see Eq. (15)] for the secondorder mass-polarization coefficient $\varepsilon_{M}^{(2)}$ (in units of $10^{-3}$ a.u.).

\begin{tabular}{|c|c|c|c|}
\hline State & Variational & Asymptotic & Difference \\
\hline $\begin{array}{r}2 P \\
3 P \\
4 P \\
5 P \\
6 P \\
7 P \\
8 P \\
9 P \\
10 P\end{array}$ & $\begin{array}{l}-86.61566(6) \\
-68.170284(4) \\
-35.64484(6) \\
-22.0071(3) \\
-14.9682(1) \\
-10.849(2) \\
-8.2302(8) \\
-6.4553(2) \\
-5.202(1)\end{array}$ & $\begin{array}{r}-54.2969 \\
-65.2006 \\
-35.4614 \\
-22.1900 \\
-15.1669 \\
-11.0129 \\
-8.3561 \\
-6.5555 \\
-5.2794\end{array}$ & $\begin{array}{l}32.3188 \\
-2.9697 \\
-0.1834 \\
0.1829(3) \\
0.1986(1) \\
0.164(2) \\
0.1258(8) \\
0.1001(2) \\
0.078(1)\end{array}$ \\
\hline $\begin{array}{r}3 D \\
4 D \\
5 D \\
6 D \\
7 D \\
8 D \\
9 D \\
10 D\end{array}$ & $\begin{array}{c}-55.96951(2) \\
-31.44940(2) \\
-20.1081(2) \\
-13.9533(3) \\
-10.24515(3) \\
-7.8412(4) \\
-6.1931(1) \\
-5.0152(9)\end{array}$ & $\begin{array}{r}-55.94(2) \\
-31.43(1) \\
-20.098(7) \\
-13.947(4) \\
-10.241(3) \\
-7.838(2) \\
-6.191(1) \\
-5.013(1)\end{array}$ & $\begin{array}{l}-0.03(2) \\
-0.02(1) \\
-0.011(7) \\
-0.006(4) \\
-0.004(3) \\
-0.004(2) \\
-0.003(1) \\
-0.002(1)\end{array}$ \\
\hline $\begin{array}{r}4 F \\
5 F \\
6 F \\
7 F \\
8 F \\
9 F \\
10 F\end{array}$ & $\begin{array}{c}-31.276164(4) \\
-20.015030(7) \\
-13.89810(3) \\
-10.2099(4) \\
-7.8165(3) \\
-6.17561(1) \\
-5.00207(2)\end{array}$ & $\begin{array}{r}-31.2761(1) \\
-20.01496(9) \\
-13.89805(6) \\
10.21004(4) \\
-7.81657(3) \\
-6.17574(2) \\
-5.00214(2)\end{array}$ & $\begin{array}{c}-0.0000(1) \\
-0.00007(9) \\
-0.00005(7) \\
0.0001(4) \\
0.0001(3) \\
0.00013(3) \\
0.00006(2)\end{array}$ \\
\hline $\begin{array}{r}5 G \\
6 G \\
7 G \\
8 G \\
9 G \\
10 G\end{array}$ & $\begin{array}{c}-20.00356272(7) \\
-13.89118(1) \\
-10.20561(7) \\
-7.813566(3) \\
-6.173586(4) \\
-5.00055(3)\end{array}$ & $\begin{array}{r}-20.003568(3) \\
-13.891183(3) \\
-10.205613(2) \\
-7.813564(2) \\
-6.173605(1) \\
-5.000568(1)\end{array}$ & $\begin{array}{c}0.000005(3) \\
0.00000(1) \\
0.00000(7) \\
-0.000002(3) \\
0.000019(5) \\
0.00002(3)\end{array}$ \\
\hline $\begin{array}{r}6 H \\
7 H \\
8 H \\
9 H \\
10 H\end{array}$ & $\begin{array}{l}-13.88961902(4) \\
-10.204589(2) \\
-7.812857(6) \\
-6.173103(3) \\
-5.0001935(2)\end{array}$ & $\begin{array}{r}-13.88961936(7) \\
-10.20458849(8) \\
-7.81285993(7) \\
-6.17310212(6) \\
-5.00019656(5)\end{array}$ & $\begin{array}{c}0.00000034(8) \\
-0.000000(2) \\
0.000003(6) \\
-0.000000(3) \\
0.0000031(2)\end{array}$ \\
\hline $\begin{array}{r}7 I \\
8 I \\
9 I \\
10 I\end{array}$ & $\begin{array}{c}-10.20427676(2) \\
-7.81264291(4) \\
-6.1729459(2) \\
-5.000081(1)\end{array}$ & $\begin{array}{r}-10.204276794(4) \\
-7.812642992(5) \\
-6.172945836(5) \\
-5.000080594(4)\end{array}$ & $\begin{array}{r}0.00000004(2) \\
0.00000008(4) \\
-0.0000001(2) \\
-0.000000(1)\end{array}$ \\
\hline $\begin{array}{r}8 K \\
9 K \\
10 K\end{array}$ & $\begin{array}{l}-7.81256302(1) \\
-6.17288759(1) \\
-5.00003688(5)\end{array}$ & $\begin{array}{l}-7.8125630145(4) \\
-6.1728875893(5) \\
-5.0000370503(5)\end{array}$ & $\begin{array}{r}-0.00000001(1) \\
-0.00000000(1) \\
0.00000017(5)\end{array}$ \\
\hline
\end{tabular}




$$
Q=\frac{1}{4 \pi}\left(\left\langle x^{-3}\right\rangle_{n L}+3 Z^{-2}\left\langle x^{-5}\right\rangle_{n L}\right)
$$

where the second term follows simply from a multipole expansion of $1 /|\mathbf{r}-\mathbf{x}|^{3}$. In the LRI picture, substituting the leading term of (119) in (118) gives the second term in

$$
\Delta V_{\text {ret }}=\frac{\alpha^{2}}{Z^{2}}\left\langle x^{-4}\right\rangle_{n L}-\frac{7 \alpha^{3}}{6 \pi}\left\langle x^{-3}\right\rangle_{n L},
$$

which is the short-range form of the asymptotic expansion for the retardation terms [9-11] (see Ref. [5] for a full discussion). The leading term is related to $\left\langle B_{2}\right\rangle$ through Eq. (77).

The spin-dependent matrix elements are listed in
Tables XIV-XVII. In each case, the matrix element refers to the $J=L$ component of the triplet. The $J=L \pm 1$ components are obtained from the tabulated quantities according to

$$
\begin{aligned}
& \left\langle n{ }^{3} L_{J}\left|B_{3}\right| n{ }^{3} L_{J}\right\rangle=t_{L}(J)\left\langle n{ }^{3} L_{L}\left|B_{3}\right| n{ }^{3} L_{L}\right\rangle, \\
& \left\langle n{ }^{3} L_{J}\left|\Delta_{3}\right| n{ }^{3} L_{J}\right\rangle=t_{L}(J)\left\langle n{ }^{3} L_{L}\left|\Delta_{3}\right| n{ }^{3} L_{L}\right\rangle, \\
& \left\langle n{ }^{3} L_{J}\left|B_{5}\right| n{ }^{3} L_{J}\right\rangle=S_{L}(J) t_{L}(J)\left\langle n^{3} L_{L}\left|B_{5}\right| n{ }^{3} L_{L}\right\rangle,
\end{aligned}
$$

\begin{tabular}{|c|c|c|c|c|}
\hline State & $p_{4}\left(n^{1} L\right)$ & $p_{4}^{\mathrm{X}}\left(n^{1} L\right)$ & $p_{4}\left(n^{3} L\right)$ & $p_{4}^{\mathrm{X}}\left(n^{3} L\right)$ \\
\hline $2 S$ & $-279.668907(5)$ & $40.057(3)$ & $-458.88520460(2)$ & $1.11852(6)$ \\
\hline $\begin{array}{r}2 P \\
3 P \\
4 P \\
5 P \\
6 P \\
7 P \\
8 P \\
9 P \\
10 P\end{array}$ & $\begin{array}{c}-29.251357(5) \\
-11.372887(6) \\
-5.316142(5) \\
-2.8777129(6) \\
-1.7251175(3) \\
-1.113216(2) \\
-0.759251(2) \\
-0.540617(1) \\
-0.398414(1)\end{array}$ & $\begin{array}{c}-262.5850(7) \\
-71.3197(3) \\
-29.2556(3) \\
-14.827(7) \\
-8.555(1) \\
-5.37(2) \\
-3.61(1) \\
-2.542(1) \\
-1.86(1)\end{array}$ & $\begin{array}{l}87.90628433(5) \\
21.92178(2) \\
8.420154(3) \\
4.071218(4) \\
2.267379(2) \\
1.389223(3) \\
0.9117219(7) \\
0.6301753(4) \\
0.4535629(4)\end{array}$ & $\begin{array}{c}472.6405(2) \\
85.5629(5) \\
28.446(2) \\
12.783(6) \\
6.824(2) \\
4.06(2) \\
2.65(2) \\
1.798(3) \\
1.284(2)\end{array}$ \\
\hline $\begin{array}{r}3 D \\
4 D \\
5 D \\
6 D \\
7 D \\
8 D \\
9 D \\
10 D\end{array}$ & $\begin{array}{l}-1.682462(2) \\
-1.1647169(3) \\
-0.73829278(4) \\
-0.4825545(4) \\
-0.3289251(2) \\
-0.23299711(4) \\
-0.1705743(5) \\
-0.128397(5)\end{array}$ & $\begin{array}{l}4.2072(3) \\
2.18(1) \\
1.222(3) \\
0.7161(1) \\
0.472(1) \\
0.3155(6) \\
0.237(6) \\
0.11(4)\end{array}$ & $\begin{array}{l}-1.413358(2) \\
-1.01256312(2) \\
-0.651406(1) \\
-0.429472(1) \\
-0.294438673(7) \\
-0.2094339(5) \\
-0.1538036(4) \\
-0.116059(2)\end{array}$ & $\begin{array}{l}-1.0355(7) \\
-0.804(5) \\
-0.4943(1) \\
-0.331(8) \\
-0.206(1) \\
-0.148(8) \\
-0.096(4) \\
-0.10(1)\end{array}$ \\
\hline $\begin{array}{r}4 F \\
5 F \\
6 F \\
7 F \\
8 F \\
9 F \\
10 F\end{array}$ & $\begin{array}{l}-0.6770027(3) \\
-0.4915782(6) \\
-0.3407643(2) \\
-0.2400217(5) \\
-0.17361282(8) \\
-0.1289514(2) \\
-0.0981072(7)\end{array}$ & $\begin{array}{l}0.148(7) \\
0.082(5) \\
0.049(4) \\
0.032(2) \\
0.021(2) \\
0.015(1) \\
0.011(1)\end{array}$ & $\begin{array}{l}-0.67644781(7) \\
-0.4911123(9) \\
-0.34043221(4) \\
-0.23978661(5) \\
-0.1734437(2) \\
-0.12882714(3) \\
-0.0980130(4)\end{array}$ & $\begin{array}{l}0.153(7) \\
0.087(5) \\
0.052(4) \\
0.034(2) \\
0.023(2) \\
0.016(1) \\
0.012(1)\end{array}$ \\
\hline $\begin{array}{r}5 G \\
6 G \\
7 G \\
8 G \\
9 G \\
10 G\end{array}$ & $\begin{array}{l}-0.27626892(3) \\
-0.2169693(2) \\
-0.1623681(5) \\
-0.1217248(1) \\
-0.0925735620(9) \\
-0.071620616(4)\end{array}$ & $\begin{array}{l}0.01636(2) \\
0.01007(2) \\
0.00641(2) \\
0.00423(2) \\
0.00288(1) \\
0.00186(1)\end{array}$ & $\begin{array}{l}-0.276268027(2) \\
-0.2169687(3) \\
-0.1623686(8) \\
-0.1217239(2) \\
-0.092573040(3) \\
-0.071620320(6)\end{array}$ & $\begin{array}{l}0.01637(2) \\
0.01008(2) \\
0.00641(2) \\
0.00423(2) \\
0.00288(1) \\
0.00198(2)\end{array}$ \\
\hline $\begin{array}{r}6 H \\
7 H \\
8 H \\
9 H \\
10 H\end{array}$ & $\begin{array}{l}-0.1289236(4) \\
-0.10705552(3) \\
-0.08472680(3) \\
-0.0666174(5) \\
-0.0527125(1)\end{array}$ & $\begin{array}{c}0.0007097(6) \\
0.000313(1) \\
0.000061(4) \\
-0.0000964(6) \\
-0.000172(2)\end{array}$ & $\begin{array}{l}-0.1289235(3) \\
-0.10705548(3) \\
-0.08472683(3) \\
-0.0666170(3) \\
-0.0527127(1)\end{array}$ & $\begin{array}{c}0.0007097(6) \\
0.0003130(7) \\
0.000062(5) \\
-0.000094(1) \\
-0.0001753(5)\end{array}$ \\
\hline $\begin{array}{r}7 I \\
8 I \\
9 I \\
10 I\end{array}$ & $\begin{array}{l}-0.06738460(2) \\
-0.05817827(2) \\
-0.04798427(8) \\
-0.03913633(2)\end{array}$ & $\begin{array}{l}-0.001602(1) \\
-0.001274(2) \\
-0.0010490(2) \\
-0.00088554(5)\end{array}$ & $\begin{array}{l}-0.06738460(2) \\
-0.05817827(5) \\
-0.04798427(6) \\
-0.03913632(2)\end{array}$ & $\begin{array}{l}-0.0016017(6) \\
-0.00127208(5) \\
-0.00104926(5) \\
-0.0008846(3)\end{array}$ \\
\hline $\begin{array}{r}8 K \\
9 K \\
10 K\end{array}$ & $\begin{array}{l}-0.038430866(6) \\
-0.0341221966(7) \\
-0.029034706(6)\end{array}$ & $\begin{array}{l}-0.00176399(3) \\
-0.00140702(2) \\
-0.001167(4)\end{array}$ & $\begin{array}{l}-0.038430866(5) \\
-0.034122194(1) \\
-0.029034709(6)\end{array}$ & $\begin{array}{l}-0.00176398(2) \\
-0.00140704(2) \\
-0.00115(2)\end{array}$ \\
\hline
\end{tabular}

where $t_{L}(L)=1$,

$$
t_{L}(J)=\frac{1}{2} \mp\left(L+\frac{1}{2}\right)
$$

TABLE V. Variational matrix elements $-\left\langle n L\left|p_{1}^{4}+p_{2}^{4}\right| n L\right\rangle / 8+10=p_{4}+(\mu / M) p_{4}^{\mathrm{X}}$ (in units of $10^{-3}$ a.u.). 
TABLE VI. Variational matrix elements $\pi\left\langle\delta\left(\mathbf{r}_{1}\right)\right\rangle-4=d_{1}+(\mu / M) d_{1}^{\mathrm{X}}$ (in units of $10^{-3}$ a.u.).

\begin{tabular}{ccccc}
\hline State & $d_{1}\left(n^{1} L\right)$ & $d_{1}^{\mathrm{X}}\left(n^{1} L\right)$ & $d_{1}\left(n^{3} L\right)$ & $d_{1}^{\mathrm{X}}\left(n^{3} L\right)$ \\
\hline $2 S$ & $113.792370(1)$ & $-1.26231(5)$ & $148.017828713(3)$ & $6.83886(4)$ \\
$2 P$ & $3.623328(3)$ & $123.7748(3)$ & $-45.172772175(1)$ & $-225.20782(8)$ \\
$3 P$ & $1.218155(3)$ & $35.4318(1)$ & $-12.936273(9)$ & $-45.9080(2)$ \\
$4 P$ & $0.522308(2)$ & $14.7401(1)$ & $-5.344949(2)$ & $-16.2423(6)$ \\
$5 P$ & $0.2684188(3)$ & $7.500(3)$ & $-2.702571(2)$ & $-7.554(2)$ \\
$6 P$ & $0.1555194(1)$ & $4.3277(1)$ & $-1.5512150(9)$ & $-4.1188(8)$ \\
$7 P$ & $0.097985(1)$ & $2.72(1)$ & $-0.971255(1)$ & $-2.49(1)$ \\
$8 P$ & $0.065659(1)$ & $1.822(6)$ & $-0.64790800(5)$ & $-1.628(4)$ \\
$9 P$ & $0.0461228(6)$ & $1.2805(5)$ & $-0.4535665(2)$ & $-1.117(1)$ \\
$10 P$ & $0.0336288(6)$ & $0.934(6)$ & $-0.32979899(2)$ & $-0.8012(9)$ \\
$3 D$ & $-0.437798(1)$ & $-1.8161(1)$ & $-0.5397661(8)$ & $0.3347(2)$ \\
$4 D$ & $-0.2000157(2)$ & $-0.944(7)$ & $-0.257910150(7)$ & $0.287(3)$ \\
$5 D$ & $-0.10607339(1)$ & $-0.528(2)$ & $-0.1391815(6)$ & $0.1805(1)$ \\
$6 D$ & $-0.0625417(2)$ & $-0.3136(1)$ & $-0.0827791(5)$ & $0.120(4)$ \\
$7 D$ & $-0.0398244(1)$ & $-0.2050(5)$ & $-0.052974123(3)$ & $0.0763(5)$ \\
$8 D$ & $-0.02687038(1)$ & $-0.1381(3)$ & $-0.0358551(3)$ & $0.054(4)$ \\
$9 D$ & $-0.0189636(2)$ & $-0.101(3)$ & $-0.0253579(2)$ & $0.036(2)$ \\
$10 D$ & $-0.013874(2)$ & $-0.06(2)$ & $-0.0185782(9)$ & $0.034(6)$ \\
\hline
\end{tabular}

for $J=L \pm 1$, and $S_{L}(J)$ is given by Eq. (105). The offdiagonal matrix elements $n^{3} L_{L}-n{ }^{1} L_{L}$ are also tabulated. It is evident from the tables that the asymptotic limits contained in Eqs. (106) and (107) are satisfied.

For all the above terms, the corrections to the leading asymptotic values given in Eqs. (106) and (107) arise from short-range effects involving overlap integrals with the inner $1 s$ electron $[36,40]$, rather than long-range polarization terms proportional to $\left\langle x^{-4}\right\rangle_{n L}$. Since the shortrange effects decrease exponentially with $L$, the leading asymptotic term alone rapidly improves in accuracy as illustrated previously for the $n=10$ states of helium (see

TABLE VII. Variational matrix elements $\pi\left\langle\delta\left(\mathbf{r}_{12}\right)\right\rangle=d_{12}+(\mu / M) d_{12}^{\mathrm{X}}$ (in units of $10^{-6}$ a.u.).

\begin{tabular}{|c|c|c|c|c|c|}
\hline State & $d_{12}\left(n^{1} L\right)$ & $d_{12}^{\mathrm{X}}\left(n^{1} L\right)$ & State & $d_{12}\left(n^{1} L\right)$ & $d_{12}^{\mathrm{X}}\left(n^{1} L\right)$ \\
\hline $2 S$ & $27169.868(4)$ & $5550 .(3)$ & $\begin{array}{l}5 G \\
6 G\end{array}$ & $\begin{array}{l}0.000015868(4) \\
0.000017666(1)\end{array}$ & $\begin{array}{l}-0.0001283(2) \\
-0.00015(3)\end{array}$ \\
\hline $2 P$ & $2309.6018(8)$ & $-10854.41(1)$ & $7 G$ & $0.000015209(2)$ & $-0.0002(1)$ \\
\hline $3 P$ & $791.729(2)$ & $-3367.130(2)$ & $8 G$ & $0.000012211(1)$ & $-0.00009164(1)$ \\
\hline $4 P$ & $350.176(2)$ & $-1419.69(2)$ & $9 G$ & $0.000009635(3)$ & $-0.000064(2)$ \\
\hline $5 P$ & $183.2091(1)$ & $-723 .(1)$ & $10 G$ & $0.000007607(3)$ & $-0.000046(5)$ \\
\hline $6 P$ & $107.28331(8)$ & $-415.2(8)$ & & & \\
\hline $7 P$ & $68.04856(2)$ & $-256.14(6)$ & $6 H$ & $0.00000001371(1)$ & $-0.0000001864(1)$ \\
\hline $8 P$ & $45.8041(1)$ & $-171.3(9)$ & $7 H$ & $0.000000018886(4)$ & $-0.00000007(8)$ \\
\hline $9 P$ & $32.27624(8)$ & $-121 .(2)$ & $8 H$ & $0.00000001891(3)$ & $-0.0000002(5)$ \\
\hline \multirow[t]{2}{*}{$10 P$} & $23.5858(4)$ & $-90 .(2)$ & $9 H$ & $0.00000001700(9)$ & $-0.000000139(1)$ \\
\hline & & & $10 \mathrm{H}$ & $0.000000014481(8)$ & $-0.0000001(2)$ \\
\hline $3 D$ & $7.18637(1)$ & $-30.59(8)$ & & & \\
\hline $4 D$ & $4.23074(8)$ & $-17.787(6)$ & $7 I$ & $0.0000000000093(2)$ & $-0.0000000003(2)$ \\
\hline $5 D$ & $2.46113(4)$ & $-10.333(6)$ & $8 I$ & $0.0000000000152(4)$ & $-0.0000000010(7)$ \\
\hline $6 D$ & $1.51851(2)$ & $-6.17(5)$ & $9 I$ & $0.0000000000174(4)$ & $-0.0000000008(3)$ \\
\hline $7 D$ & $0.99234(8)$ & $-4.10(7)$ & $10 I$ & $0.0000000000174(4)$ & $0.000000000(3)$ \\
\hline $8 D$ & $0.68057(5)$ & $-2.9(2)$ & & & \\
\hline $9 D$ & $0.48558(2)$ & $-2.08(6)$ & & & \\
\hline $10 D$ & $0.35798(1)$ & $-1.36(7)$ & & & \\
\hline $4 F$ & $0.013053(1)$ & $-0.0827(9)$ & & & \\
\hline $5 F$ & $0.0110798(7)$ & $-0.065289(1)$ & & & \\
\hline $6 F$ & $0.0080014(2)$ & $-0.047(2)$ & & & \\
\hline $7 F$ & $0.00568746(1)$ & $-0.034(1)$ & & & \\
\hline $8 F$ & $0.0041041(4)$ & $-0.025(1)$ & & & \\
\hline $9 F$ & $0.0030280(6)$ & $-0.020(2)$ & & & \\
\hline $10 F$ & $0.0022847(7)$ & $-0.014(1)$ & & & \\
\hline
\end{tabular}


Table 19 of Ref. [5]). As a consequence, high accuracy can be expected from multiconfiguration Hartree-Fock calculations for these terms $[41,42]$. However, the same is not true for the spin-independent terms where polarization effects are important.

The spin-dependent matrix elements for the $2{ }^{3} P_{J}$ states are of particular interest because comparisons with high-precision measurements of the fine-structure splittings may eventually lead to an atomic-physics value for the fine-structure constant. This was the motivation for a long sequence of calculations beginning with Schwartz [43], continuing with the operators for the higher-order spin-dependent terms derived by Douglas and Kroll [44], and culminating with the second-order terms evaluated by Lewis and Serafino [31]. However, the accuracy of neither theory nor experiment was sufficient to compete with $\alpha$ obtained from the quantum Hall effect or the electron magnetic moment [45]. The convergence study presented in Table XVIII shows that the lowest-order matrix elements are now known to an accuracy of about 3 parts in $10^{9}$ for the sum of the three terms. This improves by 3 orders of magnitude the previous results of Schwartz [43], who evidently overestimated the accuracy of his calculation (see the table). In the present work, the entire amount of the extrapolation is taken as a conservative estimate of the uncertainty.

Further work is in progress to achieve a similar improvement in the higher-order corrections. Lewis and Serafino [31] have considered all contributions to the fine-structure splittings up to order $\alpha^{4}$ a.u. To this order, self-energy and vacuum-polarization effects are spin independent, and so do not contribute. However, self-

TABLE VIII. Comparison of spin-averaged variational matrix elements with the asymptotic values [see Eq. (62)] for $\left\langle B_{1}+B_{4}\right\rangle$ (in units of MHz, with $\alpha^{2} e^{2} / a_{\mu}=350329.1022 \pm 0.031 \mathrm{MHz}$ ).

\begin{tabular}{|c|c|c|c|c|c|}
\hline State & Variational & $U_{1}^{\mathrm{a}}$ & $U_{2}^{\mathrm{b}}$ & $U_{1}+U_{2}$ & Difference \\
\hline $\begin{array}{r}3 D \\
4 D \\
5 D \\
6 D \\
7 D \\
8 D \\
9 D \\
10 D\end{array}$ & $\begin{array}{c}-884.747(1) \\
-541.8070(1) \\
-329.3458(4) \\
-210.6648(4) \\
141.70124(8) \\
-99.4728(2) \\
-72.3466(2) \\
-54.189(2)\end{array}$ & $\begin{array}{r}-875.024601 \\
-536.491751 \\
-326.370636 \\
-208.877304 \\
-140.555999 \\
-98.699621 \\
-71.801756 \\
-53.792219\end{array}$ & $\begin{array}{r}-17.480413 \\
-10.060415 \\
-5.773710 \\
-3.525309 \\
-2.284442 \\
-1.555854 \\
-1.103714 \\
-0.809667\end{array}$ & $\begin{array}{l}-893(14) \\
-547(8) \\
-332(5) \\
-212(3) \\
-143(2) \\
-100(1) \\
-72.9(9) \\
-54.6(7)\end{array}$ & $\begin{array}{l}8(14) \\
5(8) \\
3(5) \\
2(3) \\
1(2) \\
1(1) \\
0.6(9) \\
0.4(7)\end{array}$ \\
\hline $\begin{array}{r}4 F \\
5 F \\
6 F \\
7 F \\
8 F \\
9 F \\
10 F\end{array}$ & $\begin{array}{c}-261.80289(9) \\
-186.2456(4) \\
-127.94586(6) \\
-89.6500(2) \\
-64.62214(7) \\
-47.88009(7) \\
-36.3597(3)\end{array}$ & $\begin{array}{r}-261.293690 \\
-185.884412 \\
-127.705314 \\
-89.486953 \\
-64.508075 \\
-47.797871 \\
-36.298548\end{array}$ & $\begin{array}{l}-0.479545 \\
-0.343251 \\
-0.229536 \\
-0.155930 \\
-0.109177 \\
-0.078818 \\
-0.058500\end{array}$ & $\begin{array}{r}-261.77(9) \\
-186.23(7) \\
-127.93(4) \\
-89.64(3) \\
-64.62(2) \\
-47.88(2) \\
-36.36(1)\end{array}$ & $\begin{array}{l}-0.03(9) \\
-0.02(7) \\
-0.01(4) \\
-0.01(3) \\
-0.00(2) \\
-0.00(2) \\
-0.00(1)\end{array}$ \\
\hline $\begin{array}{r}5 G \\
6 G \\
7 G \\
8 G \\
9 G \\
10 G\end{array}$ & $\begin{array}{l}-100.20873(1) \\
-78.2077(1) \\
-58.3479(3) \\
-43.66103(8) \\
-33.162870(1) \\
-25.633080(3)\end{array}$ & $\begin{array}{r}-100.171288 \\
-78.177231 \\
-58.325471 \\
-43.644573 \\
-33.150664 \\
-25.623809\end{array}$ & $\begin{array}{l}-0.035532 \\
-0.028672 \\
-0.021194 \\
-0.015547 \\
-0.011550 \\
-0.008737\end{array}$ & $\begin{array}{c}-100.207(3) \\
-78.206(3) \\
-58.347(2) \\
-43.660(1) \\
-33.162(1) \\
-25.6325(8)\end{array}$ & $\begin{array}{l}-0.002(3) \\
-0.002(3) \\
-0.001(2) \\
-0.001(1) \\
-0.001(1) \\
-0.0005(8)\end{array}$ \\
\hline $\begin{array}{r}6 H \\
7 H \\
8 H \\
9 H \\
10 H\end{array}$ & $\begin{array}{l}-45.8691(2) \\
-37.99242(1) \\
-30.02850(1) \\
-23.5904(2) \\
-18.65572(6)\end{array}$ & $\begin{array}{l}-45.864402 \\
-37.988312 \\
-30.025238 \\
-23.587765 \\
-18.653703\end{array}$ & $\begin{array}{l}-0.004557 \\
-0.003954 \\
-0.003133 \\
-0.002433 \\
-0.001893\end{array}$ & $\begin{array}{l}-45.8690(2) \\
-37.9923(2) \\
-30.0284(2) \\
-23.5902(1) \\
-18.6556(1)\end{array}$ & $\begin{array}{l}-0.0002(3) \\
-0.0002(2) \\
-0.0001(2) \\
-0.0002(2) \\
-0.0001(1)\end{array}$ \\
\hline $\begin{array}{r}7 I \\
8 I \\
9 I \\
10 I\end{array}$ & $\begin{array}{l}-23.79482(1) \\
-20.51926(2) \\
-16.91266(3) \\
-13.78819(1)\end{array}$ & $\begin{array}{l}-23.793961 \\
-20.518484 \\
-16.912000 \\
-13.787661\end{array}$ & $\begin{array}{l}-0.000833 \\
-0.000757 \\
-0.000631 \\
-0.000512\end{array}$ & $\begin{array}{l}-23.79479(3) \\
-20.51924(3) \\
-16.91263(2) \\
-13.78817(2)\end{array}$ & $\begin{array}{l}-0.00002(3) \\
-0.00002(3) \\
-0.00003(2) \\
-0.00001(2)\end{array}$ \\
\hline $\begin{array}{r}8 K \\
9 K \\
10 K\end{array}$ & $\begin{array}{l}-13.524110(3) \\
-12.0002753(5) \\
-10.207353(3)\end{array}$ & $\begin{array}{l}-13.523911 \\
-12.000090 \\
-10.207192\end{array}$ & $\begin{array}{l}-0.000195 \\
-0.000183 \\
-0.000158\end{array}$ & $\begin{array}{l}-13.524106(7) \\
-12.000273(7) \\
-10.207351(6)\end{array}$ & $\begin{array}{l}-0.000004(6) \\
-0.000002(5) \\
-0.000003(5)\end{array}$ \\
\hline
\end{tabular}

${ }^{\mathrm{a}} U_{1}=h_{1}(n L)+\Delta B_{1}\left(\alpha_{\mathrm{rel}}\right)$.

${ }^{\mathrm{b}} U_{2}=\Delta B_{1}\left(\phi_{1}\right)$ [see Eqs. (62)-(65)]. 
energy terms of order $\alpha^{5} Z^{6} \ln (Z \alpha)^{-2}$ in the one-electron Lamb shift [46-48] become spin dependent for $p$ states. The spin dependence follows from the fact that the small component of the $p_{1 / 2}$ state is $s$-like, and so does not vanish at the origin, while the small component of $p_{3 / 2}$ is $d$ like which does vanish at the origin. The spin dependence carries over to the two-electron case where, in an unscreened hydrogenic approximation, it contributes at the $\pm 0.5-\mathrm{MHz}$ level of accuracy [5]. This is undoubtedly an overestimate, but a proper two-electron treatment will be required for a high-precision comparison with experimental fine-structure splittings in helium.
C. Total energies

The main purpose of this work is to present a tabulation of energy levels for all states of helium up to $n=10$ which systematically includes all contributions of orders $\alpha^{2}, \alpha^{3}, \alpha^{2} \mu / M,(\mu / M)^{2}$, and $\alpha^{3} \mu / M$. Because of the nonperturbative method of treating mass polarization, the results actually contain terms of all orders in $\mu / M$, along with the major part of the $(\alpha \mu / M)^{2}$ term. Collecting the results of the preceding sections, the total energy is

TABLE IX. Variational matrix elements $\left\langle B_{2}\right\rangle=b_{2}+(\mu / M) b_{2}^{\mathrm{X}}$ (in units of $10^{-3} \alpha^{2}$ a.u.).

\begin{tabular}{|c|c|c|c|c|}
\hline State & $b_{2}\left(n^{1} L\right)$ & $b_{2}^{\mathrm{X}}\left(n^{1} L\right)$ & $b_{2}\left(n^{3} L\right)$ & $b_{2}^{\mathrm{X}}\left(n^{3} L\right)$ \\
\hline $2 S$ & $-9.25304619(5)$ & $142.25741(4)$ & $-1.628430061(2)$ & $23.5281985(5)$ \\
\hline $\begin{array}{r}2 P \\
3 P \\
4 P \\
5 P \\
6 P \\
7 P \\
8 P \\
9 P \\
10 P\end{array}$ & $\begin{array}{l}-20.33047410(2) \\
-6.69736894(7) \\
-2.91827835(6) \\
-1.516084310(6) \\
-0.884359852(3) \\
-0.5596288972(3) \\
-0.376116535(8) \\
-0.264755498(5) \\
-0.19332591(2)\end{array}$ & $\begin{array}{c}104.505483(2) \\
35.4110266(5) \\
15.8153614(7) \\
8.36976(3) \\
4.95067(2) \\
3.16644(1) \\
2.14628(7) \\
1.52115(5) \\
1.1171(1)\end{array}$ & $\begin{array}{l}35.08088683(4) \\
10.344716185(4) \\
4.3093369880(9) \\
2.186478863(2) \\
1.2572374418(6) \\
0.788014456(2) \\
0.52602275(3) \\
0.368407518(3) \\
0.267963890(5)\end{array}$ & $\begin{array}{c}152.372647(1) \\
42.7319904(3) \\
17.8571713(1) \\
9.148602(1) \\
5.309512(3) \\
3.35420(7) \\
2.2535(2) \\
1.586935(1) \\
1.159526(2)\end{array}$ \\
\hline $\begin{array}{r}3 D \\
4 D \\
5 D \\
6 D \\
7 D \\
8 D \\
9 D \\
10 D\end{array}$ & $\begin{array}{l}0.1216490302(4) \\
0.055448022(9) \\
0.029361923(2) \\
0.0172962694(8) \\
0.011007287(7) \\
0.007423938(5) \\
0.005238034(1) \\
0.0038309874(6)\end{array}$ & $\begin{array}{l}10.925689(2) \\
6.04559(1) \\
3.526993(1) \\
2.20524(1) \\
1.461824(2) \\
1.01578(1) \\
0.73323(1) \\
0.5460017(8)\end{array}$ & $\begin{array}{l}0.1322794995(6) \\
0.061389771(6) \\
0.0327326860217(2) \\
0.0193470963(4) \\
0.0123360114(3) \\
0.00833002781(9) \\
0.00588199656(4) \\
0.00430435354(3)\end{array}$ & $\begin{array}{l}9.34509(4) \\
5.13767(9) \\
3.004600(3) \\
1.884821(3) \\
1.2531580(1) \\
0.872981(5) \\
0.631483(2) \\
0.4710922(7)\end{array}$ \\
\hline $\begin{array}{r}4 F \\
5 F \\
6 F \\
7 F \\
8 F \\
9 F \\
10 F\end{array}$ & $\begin{array}{l}0.0095026657(1) \\
0.00542077450(8) \\
0.00331048611(4) \\
0.002150350885(8) \\
0.00146902493(8) \\
0.0010454222(3) \\
0.0007692367(1)\end{array}$ & $\begin{array}{l}2.806007514(1) \\
1.837601740(2) \\
1.2180261037(1) \\
0.836551(1) \\
0.5953424(2) \\
0.4371982(7) \\
0.3298392(6)\end{array}$ & $\begin{array}{l}0.009503888432(4) \\
0.00542175271(2) \\
0.003311168732(5) \\
0.00215082582(4) \\
0.00146936272(3) \\
0.0010456691(1) \\
0.00076942145(5)\end{array}$ & $\begin{array}{l}2.8058263483(4) \\
1.83745427(1) \\
1.2179220(1) \\
0.836479(1) \\
0.59529001(7) \\
0.4371596(6) \\
0.3298087(6)\end{array}$ \\
\hline $\begin{array}{r}5 G \\
6 G \\
7 G \\
8 G \\
9 G \\
10 G\end{array}$ & $\begin{array}{l}0.0012981292938(3) \\
0.0008340203796(1) \\
0.000556538867(1) \\
0.0003864277507(1) \\
0.000277934795(7) \\
0.000206018195(2)\end{array}$ & $\begin{array}{l}1.0687501483(3) \\
0.77294170(3) \\
0.5562145(2) \\
0.407518081(8) \\
0.305274487(3) \\
0.2336610(1)\end{array}$ & $\begin{array}{l}0.00129812060498(4) \\
0.00083401075918(8) \\
0.000556530613(2) \\
0.000386421130026(5) \\
0.0002779295822(2) \\
0.000206014077(6)\end{array}$ & $\begin{array}{l}1.06875025139(2) \\
0.77294181(3) \\
0.5562146(2) \\
0.407518152(4) \\
0.3052745620(4) \\
0.23366112(5)\end{array}$ \\
\hline $\begin{array}{r}6 H \\
7 H \\
8 H \\
9 H \\
10 H\end{array}$ & $\begin{array}{l}0.000264234003215(1) \\
0.00018340680988(1) \\
0.00013024763243(2) \\
0.000095025244(1) \\
0.0000711217277(6)\end{array}$ & $\begin{array}{l}0.49143779006(2) \\
0.378919171(9) \\
0.28873498(2) \\
0.22184525(2) \\
0.1728390828(6)\end{array}$ & $\begin{array}{l}0.000264233989965(3) \\
0.000183406791504(4) \\
0.0001302476144(1) \\
0.000095025229(5) \\
0.0000711217138(3)\end{array}$ & $\begin{array}{l}0.49143779028(3) \\
0.3789191559(2) \\
0.288734984(7) \\
0.221845230(6) \\
0.1728390823(2)\end{array}$ \\
\hline $\begin{array}{r}7 I \\
8 I \\
9 I \\
10 I\end{array}$ & $\begin{array}{l}0.00007024025453(6) \\
0.00005148618650(4) \\
0.00003829088484(4) \\
0.00002902392014(8)\end{array}$ & $\begin{array}{l}0.2564128190(5) \\
0.20666070864(3) \\
0.16419970653(1) \\
0.1308144090(9)\end{array}$ & $\begin{array}{l}0.00007024025465(7) \\
0.00005148618649(5) \\
0.00003829088482(4) \\
0.0000290239201(1)\end{array}$ & $\begin{array}{l}0.2564128181(4) \\
0.2066607089(2) \\
0.1641997069(3) \\
0.130814406(4)\end{array}$ \\
\hline $\begin{array}{r}8 K \\
9 K \\
10 K\end{array}$ & $\begin{array}{l}0.000022579147920(0) \\
0.000017235050114(0) \\
0.000013281713075(1)\end{array}$ & $\begin{array}{l}0.14651923607(0) \\
0.12195925172(0) \\
0.1000205149(9)\end{array}$ & $\begin{array}{l}0.000022579147920(0) \\
0.000017235050114(0) \\
0.00001328171310(1)\end{array}$ & $\begin{array}{l}0.14651923607(0) \\
0.12195925176(5) \\
0.1000205146(1)\end{array}$ \\
\hline
\end{tabular}




$$
\begin{aligned}
E_{\mathrm{tot}}= & E_{\mathrm{NR}}+\Delta E_{M}^{(1)}+\Delta E_{M}^{(2)}+\Delta E_{\mathrm{rel}} \\
& +\Delta E_{\mathrm{anom}}+\Delta E_{s t}+\left(\Delta E_{\mathrm{RR}}\right)_{M} \\
& +\left(\Delta E_{\mathrm{RR}}\right)_{\mathrm{X}}+\Delta E_{\mathrm{nuc}}+\Delta E_{L, 1}+\Delta E_{L, 2} .
\end{aligned}
$$

The meaning of each of the terms is defined below, and, as an aid in identifying the physical effects included, each term is expressed in terms of its corresponding asymptotic expansion. All terms are expressed relative to $\mathrm{He}^{+}(1 s)$ (where applicable), so that each is a contribution to the negative ionization energy.

$E_{\mathrm{NR}}$ is the nonrelativistic energy without mass polarization, expressed in the form

$$
E_{\mathrm{NR}}=-\left(4+1 / n^{2}\right) R_{M}+\Delta E_{\mathrm{NR}},
$$

where $R_{M}=(1-y) R_{\infty}$ is the finite-mass Rydberg. To save tabulating excessively many figures, only $\Delta E_{\mathrm{NR}}$ is given in the tables. For convenience, the values of $R_{M} / n^{2}$ which must be added to $\Delta E_{\mathrm{NR}}$ and $\Delta E_{\text {tot }}$ are listed in Table XIX. The asymptotic expansion for $\Delta E_{\mathrm{NR}}$ has recently been worked out by Drachman [21] up to terms of order $\left\langle x^{-10}\right\rangle_{n L}$. For completeness, the

TABLE X. Comparison of spin-averaged variational matrix elements with the asymptotic values [see Eq. (77)] for $\left\langle B_{2}\right\rangle$, in MHz.

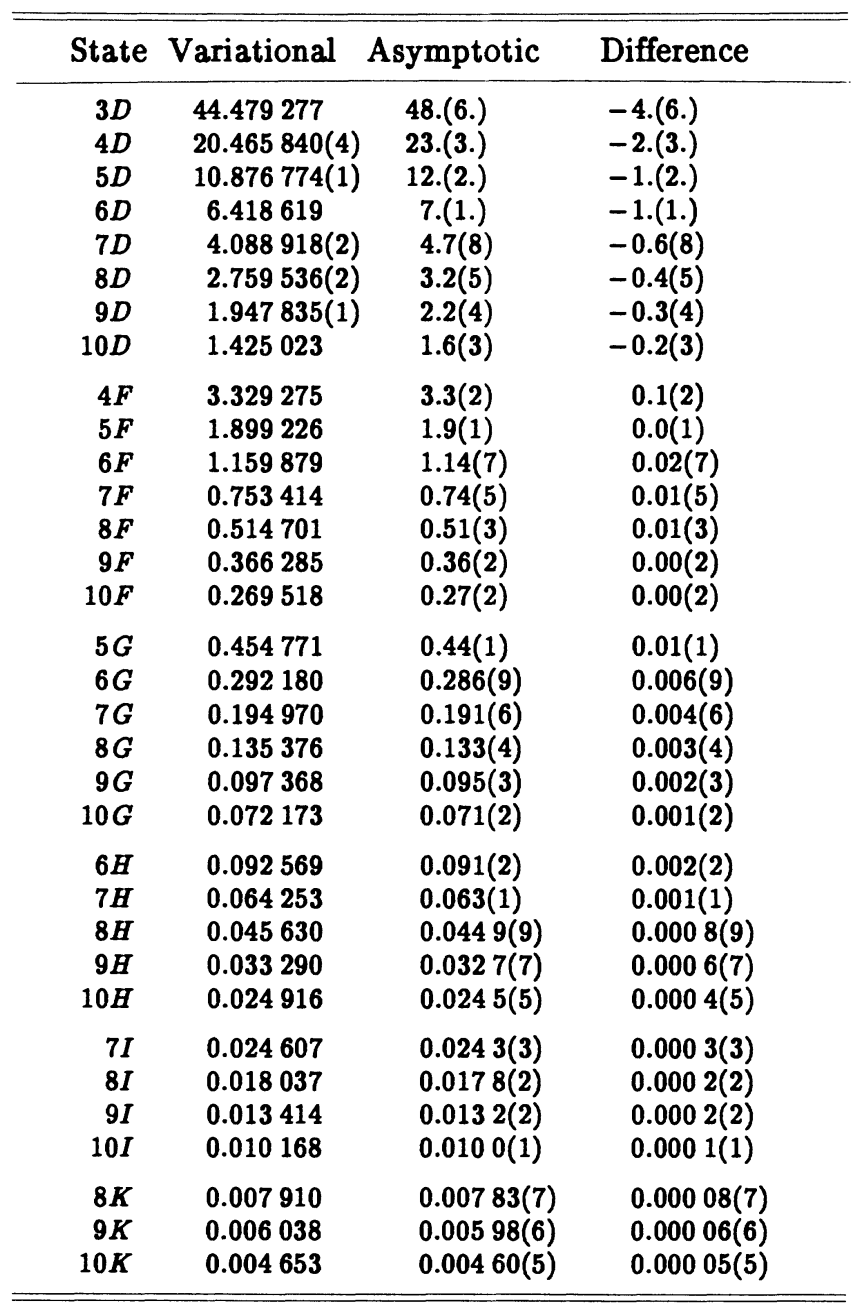

coefficients of $\frac{1}{2}\left\langle x^{-9}\right\rangle_{n L}$ and $\frac{1}{2}\left\langle x^{-10}\right\rangle_{n L}$ are [26]

$$
\begin{aligned}
& c_{9}^{(0)}=-Z^{10}\left[\frac{921873}{1008}(Z-1)+\frac{14307}{8}\right], \\
& c_{10}^{(0)}=Z^{-12}\left[-\frac{436835}{128}+\frac{33295}{4} Z^{2}+\frac{33275}{4} Z^{2} L(L+1)\right]
\end{aligned}
$$

[cf. Eq. (14)], and the total energy contains the secondorder term $\left(1-6 \beta_{2} / \alpha_{1}\right) e_{2,0}^{(1,2)}$, where $e_{2,0}^{(1,2)}$ is defined in Eq. (16), and the multiplying factor is a nonadiabatic correction.

$\Delta E_{M}^{(1)}$ and $\Delta E_{M}^{(2)}$ are the first- and second-order masspolarization corrections with asymptotic expansions corresponding to Eqs. (14) and (15) [including the small $y$ dependence expressed by Eq. (115)].

$\Delta E_{\text {rel }}$ is the relativistic correction of order $\alpha^{2}$ defined by Eq. (39), with the $\mathrm{He}^{+}(1 s)$ contribution of $-\alpha^{2} Z^{4} / 8$ a.u. subtracted. From Eqs. (62), (77), and (106) the asymptotic value is thus

\begin{tabular}{|c|c|c|c|}
\hline \multicolumn{2}{|c|}{ State Variational } & \multirow{2}{*}{$\frac{\text { Asymptotic }}{11(23)}$} & \multirow{2}{*}{$\begin{array}{c}\text { Difference } \\
-5(23)\end{array}$} \\
\hline $3 D$ & $5.01(4)$ & & \\
\hline $4 D$ & $1.5(7)$ & $4(14)$ & $-3(14)$ \\
\hline $5 D$ & $0.8(1)$ & $2(8)$ & $-1(8)$ \\
\hline $6 D$ & $-0.1(4)$ & $1(5)$ & $-1(5)$ \\
\hline $7 D$ & $0.20(7)$ & $1(3)$ & $-1(3)$ \\
\hline $8 D$ & $-0.0(4)$ & $1(2)$ & $-1(2)$ \\
\hline $9 D$ & $0.3(3)$ & $0(2)$ & $-0(2)$ \\
\hline $10 D$ & $-1(2)$ & $0(1)$ & $-1(2)$ \\
\hline $4 F$ & $0.6(5)$ & $0.5(1)$ & $0.1(5)$ \\
\hline $5 F$ & $0.3(4)$ & $0.3(1)$ & $0.1(4)$ \\
\hline $6 F$ & $0.2(2)$ & $0.13(7)$ & $0.0(3)$ \\
\hline $7 F$ & $0.1(2)$ & $0.07(5)$ & $0.0(2)$ \\
\hline $8 F$ & $0.1(1)$ & $0.03(4)$ & $0.0(1)$ \\
\hline $9 F$ & $0.03(9)$ & $0.01(3)$ & $0.01(9)$ \\
\hline $10 F$ & $0.04(7)$ & $0.00(2)$ & $0.04(8)$ \\
\hline $5 G$ & $-0.137(2)$ & $-0.132(5)$ & $-0.005(5)$ \\
\hline $6 G$ & $-0.105(2)$ & $-0.101(4)$ & $-0.004(5)$ \\
\hline $7 G$ & $-0.084(1)$ & $-0.081(3)$ & $-0.003(4)$ \\
\hline $8 G$ & $-0.068(1)$ & $-0.066(2)$ & $-0.003(3)$ \\
\hline $9 G$ & $-0.0568(9)$ & $-0.055(2)$ & $-0.002(2)$ \\
\hline $10 G$ & $-0.052(1)$ & $-0.046(1)$ & $-0.006(2)$ \\
\hline $6 H$ & $-0.15752(4)$ & $-0.1571(4)$ & $-0.0005(4)$ \\
\hline $7 H$ & $-0.11757(6)$ & $-0.1171(4)$ & $-0.0004(4)$ \\
\hline $8 H$ & $-0.0910(3)$ & $-0.0909(3)$ & $-0.0001(4)$ \\
\hline $9 H$ & $-0.07309(6)$ & $-0.0727(2)$ & $-0.0003(2)$ \\
\hline $10 H$ & $-0.05958(9)$ & $-0.0595(2)$ & $-0.0000(2)$ \\
\hline $7 I$ & $-0.12832(6)$ & $-0.12822(5)$ & $-0.00010(7)$ \\
\hline $8 I$ & $-0.09874(9)$ & $-0.09864(5)$ & $-0.0001(1)$ \\
\hline $9 I$ & $-0.078325(8)$ & $-0.07828(4)$ & $-0.00005(4)$ \\
\hline $10 I$ & $-0.06367(1)$ & $-0.06364(3)$ & $-0.00003(3)$ \\
\hline $8 K$ & $-0.101313(2)$ & $-0.101298(8)$ & $-0.000014(8)$ \\
\hline $9 K$ & $-0.080228(1)$ & $-0.080216(8)$ & $-0.000012(8)$ \\
\hline $10 K$ & $-0.0555(8)$ & $-0.065093(7)$ & $-0.0004(8)$ \\
\hline
\end{tabular}

$$
\begin{gathered}
\Delta E_{\mathrm{rel}} \rightarrow h_{1}(n L)+\Delta B_{1}\left(\alpha_{\mathrm{rel}}\right)+\Delta B_{1}\left(\phi_{1}\right)+\left\langle B_{2}\right\rangle \\
+\left[Z-3+2 S_{L}(J)\right] T_{n L}(J) \delta_{S, 1} .
\end{gathered}
$$

TABLE XI. Comparison of spin-averaged variational matrix elements with the asymptotic values [see Eq. (93)] for $\left\langle B_{1}^{\mathrm{X}}+B_{4}^{\mathrm{X}}\right\rangle$, in $\mathrm{kHz}$. 
In this and the following terms, the $J$-dependent part sums to zero on taking a statistically weighted spin average of the energies.

$\Delta E_{\text {anom }}$ is the $J$-dependent part of the anomalous magnetic-moment correction [see Eq. (61)], including finite-mass contributions. From Eqs. (109) and (110), the asymptotic form is

$$
\begin{aligned}
\Delta E_{\mathrm{anom}} \rightarrow 2 \gamma_{e}\{ & Z-2+\left(2+\gamma_{e}\right) S_{L}(J) \\
& \left.-y\left[Z-2+4 S_{L}(J)\right]\right\} T_{n L}(J) .
\end{aligned}
$$

$\Delta E_{s t}$ is the singlet-triplet mixing term obtained by diagonalizing all other contributions in the $n^{3} L_{L}, n^{1} L_{L}$ two-dimensional subset of states. From Eqs. (109) and (110), the total off-diagonal matrix element is asymptotically

$$
\begin{aligned}
\left\langle{ }^{3} L_{L}\left|B_{\text {tot }}\right| n{ }^{1} L_{L}\right\rangle \rightarrow & \left(Z+1-2 y+2 \gamma_{e} Z-2 \gamma_{e} y Z\right) \\
& \times[L(L+1)]^{1 / 2} T_{n L}(J),
\end{aligned}
$$

and the diagonal singlet-triplet splitting $2 \kappa$ can be estimated to sufficient accuracy from the variational results to be

$$
2 \kappa=\left[1+\left(\frac{3}{2 L-1}\right]^{1 / 2}\right] \pi\left\langle\delta\left(\mathbf{r}_{12}\right)\right\rangle_{\text {singlet }}
$$

with $\pi\left\langle\delta\left(\mathbf{r}_{12}\right)\right\rangle$ given by Eq. (111), including $g(Z)$ (cf. Table 5 of Ref. [5]).

$\left(\Delta E_{\mathrm{RR}}\right)_{M}$ is the relativistic reduced-mass correction given by [cf. Eq. (49)]

$$
\begin{aligned}
\left(\Delta E_{\mathrm{RR}}\right)_{M}= & -y\left\langle 3\left(B_{1}+B_{4}\right)+2 B_{2}-B_{4}\right\rangle+\Delta_{2} \\
& -2 y\left\langle B_{3, e}+B_{5}+B_{6}\right\rangle+\Delta_{3} .
\end{aligned}
$$

\begin{tabular}{|c|c|c|c|c|c|}
\hline State & $\Delta_{2}\left(n^{1} L\right)$ & $\Delta_{2}\left(n^{3} L\right)$ & State & $\Delta_{2}\left(n^{1} L\right)$ & $\Delta_{2}\left(n^{3} L\right)$ \\
\hline $2 S$ & $-656.86409(1)$ & $905.33087370(4)$ & $\begin{array}{l}5 G \\
6 G\end{array}$ & $\begin{array}{l}-2.1077957(1) \\
-1.5268643(3)\end{array}$ & $\begin{array}{l}-2.10779469(1) \\
-1.526864(1)\end{array}$ \\
\hline $2 P$ & $-286.50404(2)$ & $241.31778940(9)$ & $7 G$ & $-1.099758(2)$ & $-1.099759(3)$ \\
\hline $3 P$ & $-101.71088(2)$ & $55.40139(7)$ & $8 G$ & $-0.8062474(5)$ & $-0.8062461(8)$ \\
\hline $4 P$ & $-46.11351(2)$ & $19.94744(1)$ & $9 G$ & $-0.604231276(1)$ & $-0.60423069(2)$ \\
\hline $5 P$ & $-24.555659(2)$ & $9.19357(1)$ & $10 G$ & $-0.4626418(4)$ & $-0.462640966(1)$ \\
\hline $6 P$ & $-14.5673604(6)$ & $4.937295(9)$ & & & \\
\hline $7 P$ & $-9.331936(8)$ & $2.94036(1)$ & $6 H$ & $-0.976763(2)$ & $-0.976763(1)$ \\
\hline $8 P$ & $-6.330439(9)$ & $1.886296(1)$ & $7 H$ & $-0.7536047(1)$ & $-0.7536046(1)$ \\
\hline $9 P$ & $-4.488790(6)$ & $1.279749(2)$ & $8 H$ & $-0.5744669(1)$ & $-0.5744670(1)$ \\
\hline $10 P$ & $-3.297113(9)$ & $0.9069152(8)$ & $\begin{array}{r}9 H \\
10 H\end{array}$ & $\begin{array}{l}-0.441503(2) \\
-0.3440410(5)\end{array}$ & $\begin{array}{l}-0.441502(1) \\
-0.3440404(5)\end{array}$ \\
\hline $3 D$ & $-17.271572(9)$ & $-17.493370(6)$ & & & \\
\hline $4 D$ & $-9.774938(1)$ & $-9.91158494(2)$ & $7 I$ & $-0.51119101(6)$ & $-0.51119100(9)$ \\
\hline $5 D$ & $-5.776394211(3)$ & $-5.857236(4)$ & $8 I$ & $-0.41212496(9)$ & $-0.4121250(2)$ \\
\hline $6 D$ & $-3.642282(1)$ & $-3.692528(4)$ & $9 I$ & $-0.3275105(3)$ & $-0.3275105(2)$ \\
\hline $7 D$ & $-2.428937(1)$ & $-2.46189036(1)$ & $10 I$ & $-0.26095526(7)$ & $-0.2609552(1)$ \\
\hline $8 D$ & $-1.6953596(3)$ & $-1.717997(2)$ & & & \\
\hline $9 D$ & $-1.228027(2)$ & $-1.244191(2)$ & $8 K$ & $-0.29251107(2)$ & $-0.29251107(2)$ \\
\hline $10 D$ & $-0.91702(2)$ & $-0.928949(9)$ & $\begin{array}{r}9 K \\
10 K\end{array}$ & $\begin{array}{l}-0.243516326(3) \\
-0.19973130(2)\end{array}$ & $\begin{array}{l}-0.243516321(5) \\
-0.19973130(3)\end{array}$ \\
\hline $4 F$ & $-5.398868(1)$ & $-5.3986036(3)$ & & & \\
\hline $5 F$ & $-3.554138(3)$ & $-3.553919(3)$ & & & \\
\hline $6 F$ & $-2.3622494(6)$ & $-2.3620976(2)$ & & & \\
\hline $7 F$ & $-1.625210(2)$ & $-1.6251037(5)$ & & & \\
\hline $8 F$ & $-1.1579841(2)$ & $-1.1579075(9)$ & & & \\
\hline $9 F$ & $-0.8511329(5)$ & $-0.8510766(1)$ & & & \\
\hline $10 F$ & $-0.642563(3)$ & $-0.642520(2)$ & & & \\
\hline
\end{tabular}

With the use of Eqs. (62), (77), (98), (100), and (106), the asymptotic form is thus

$$
\begin{aligned}
\left(\Delta E_{\mathrm{RR}}\right)_{M} \rightarrow & -3 y\left[h_{1}(n L)+\Delta B_{1}\left(\alpha_{\mathrm{rel}}\right)+\Delta B_{1}\left(\phi_{1}\right)\right]+y\left(Z h_{2}(n L)+\frac{325}{16 Z^{2}} \alpha^{2}[1+(Z-2) / 6]\left\langle x^{-4}\right\rangle_{n L}\right) \\
& +y \alpha^{2}\left(-\frac{31}{4 Z^{2}}\left\langle x^{-4}\right\rangle_{n L}+\frac{1447}{32 Z^{4}}\left\langle x^{-6}\right\rangle_{n L}\right)-2 y\left\{\left\langle B_{2}\right\rangle+\left[Z-3+1+2 S_{L}(J)\right] T_{n L}(J) \delta_{S, 1}\right\} .
\end{aligned}
$$

$\left(\Delta E_{\mathrm{RR}}\right)_{\mathrm{X}}$ is the relativistic-recoil cross term between relativistic operators and the mass-polarization operator given

TABLE XII. Variational matrix elements $\left\langle\Delta_{2}\right\rangle+16 \alpha^{2} \mu / M$ (in units of $10^{-3} \alpha^{2} \mu / M$ a.u.). 
by Eqs. (54) and (55). With use of Eqs. (93), (99), and (106), the asymptotic form is

$$
\begin{aligned}
\left(\Delta E_{\mathrm{RR}}\right)_{\mathrm{X}} \rightarrow & y \alpha^{2}\left[Z^{2}(Z-1) \alpha_{1, \mathrm{rel}}-\frac{20}{9 Z^{2}}+\frac{25}{16 Z^{2}}\right]\left\langle x^{-4}\right\rangle_{n L}-y h_{2}(n L) \\
& +2 y(Z-1) \Delta B_{1}\left(\phi_{1}\right)-(4 \pm 1) y \alpha^{2}\left\langle x^{-6}\right\rangle_{n L}-2 y T_{n L}(J)+y^{2}\left[-\frac{5}{12}\left[\frac{\alpha Z(Z-1)}{n}\right]^{2}+4 h_{1}(n L)\right]+\left(\delta E_{\mathrm{RR}}\right)_{\mathrm{X}} .
\end{aligned}
$$

The term $\left(\delta E_{\mathrm{RR}}\right)_{\mathrm{X}}$ is introduced because of the term defined in Eq. (116) which was not included in the variational calculations. Thus the correct value is zero, but the variational calculations correspond to the value

$$
\left(\delta E_{\mathrm{RR}}\right)_{\mathrm{X}}=\frac{1}{6} y^{2}\left(\frac{\alpha Z(Z-1)}{n}\right)^{2} .
$$

In other words, $\left(\delta E_{\mathrm{RR}}\right)_{\mathrm{X}} \simeq 4.39 / n^{2} \mathrm{kHz}$ should be subtracted from the tabulated variational energies to make them asymptotically correct. This has no effect on $\Delta n=0$ transitions, and is otherwise negligible at current levels of accuracy. The sum $\left(\Delta E_{\mathrm{RR}}\right)_{M}+\left(\Delta E_{\mathrm{RR}}\right)_{\mathrm{X}}$ is asymptotically small and nearly independent of $L$ for a given $n$, as expected from Eqs. (52) and (102).

$\Delta E_{\text {nuc }}$ is the finite-nuclear-size correction given by

$$
\begin{aligned}
\Delta E_{\mathrm{nuc}} & =\frac{2}{3} \pi Z\left(R / a_{0}\right)^{2}\left[\left\langle\delta\left(\mathrm{r}_{1}\right)+\delta\left(\mathrm{r}_{2}\right)\right\rangle-Z^{3} / \pi\right] \\
& \rightarrow \frac{2}{3}\left(R / a_{0}\right)^{2}\left[-\frac{31}{2 Z^{2}}\left\langle x^{-4}\right\rangle_{n L}+\frac{1447}{16 Z^{4}}\left\langle x^{-6}\right\rangle_{n L}\right],
\end{aligned}
$$

where $R$ is the rms nuclear radius. For ${ }^{4} \mathrm{He}$, $R=1.673 \pm 0.001 \mathrm{fm}[49]$.

$\Delta E_{L, 1}$ is the essentially one-electron part of the Lamb shift, including two-electron corrections to the Bethe logarithm and to the electron density at the nucleus. Goldman and Drake [6] have recently calculated the correction of order $\left\langle x^{-4}\right\rangle_{n L}$ in the asymptotic expansion of the Bethe logarithm due to the electric-field perturbation of

\begin{tabular}{|c|c|c|c|c|c|}
\hline State & $Q\left(n^{1} L\right)$ & $Q\left(n^{3} L\right)$ & State & $Q\left(n^{1} L\right)$ & $Q\left(n^{3} L\right)$ \\
\hline \multirow[t]{2}{*}{$2 S$} & $5.406997(6)$ & $3.092498767110(6)$ & $5 G$ & $0.007094112708(3)$ & $0.00709410981841(4)$ \\
\hline & & & $6 G$ & $0.0041083742401(6)$ & $0.00410837103898(3)$ \\
\hline $2 P$ & $3.3744964(9)$ & $3.813917912(5)$ & $7 G$ & $0.0025883211850(8)$ & $0.00258831843403(5)$ \\
\hline $3 P$ & $0.997136(3)$ & $1.037395044(4)$ & $8 G$ & $0.0017344580241(5)$ & $0.0017344558131(2)$ \\
\hline $4 P$ & $0.421013(2)$ & $0.422102578(1)$ & $9 G$ & $0.001218397474(2)$ & $0.00121839572976(4)$ \\
\hline $5 P$ & $0.2157564(2)$ & $0.211998588365(1)$ & $10 G$ & $0.000888332186(2)$ & $0.00088833080835(9)$ \\
\hline $6 P$ & $0.12495324(9)$ & $0.1212506126(1)$ & & & \\
\hline $7 P$ & $0.07873546(2)$ & $0.075758038(3)$ & $6 H$ & $0.00223543775845(0)$ & $0.002235437755820(1)$ \\
\hline $8 P$ & $0.0527720(1)$ & $0.050468431(3)$ & $7 H$ & $0.00140814206524(0)$ & $0.001408142061610(2)$ \\
\hline $9 P$ & $0.03707802(8)$ & $0.0352976936(2)$ & $8 H$ & $0.000943519269346(1)$ & $0.000943519265740(3)$ \\
\hline \multirow[t]{2}{*}{$10 P$} & $0.0270389(4)$ & $0.0256489441(5)$ & $9 \boldsymbol{H}$ & $0.000662747169313(0)$ & $0.00066274716606(5)$ \\
\hline & & & $10 \mathrm{H}$ & $0.000483186017671(1)$ & $0.000483186014925(1)$ \\
\hline $3 D$ & $0.20596601(1)$ & $0.20527901703(3)$ & & & \\
\hline $4 D$ & $0.08766307(7)$ & $0.0872413326(7)$ & $7 I$ & $0.000850311944538(0)$ & $0.000850311944532(6)$ \\
\hline $5 D$ & $0.04505499(3)$ & $0.0448040167(2)$ & $8 I$ & $0.000569717408995(0)$ & $0.000569717408993(0)$ \\
\hline $6 D$ & $0.02612508(1)$ & $0.0259679777(1)$ & $9 I$ & $0.000400166633183(1)$ & $0.000400166633180(1)$ \\
\hline $7 D$ & $0.01647086(7)$ & $0.0163671582(9)$ & $10 I$ & $0.000291740127913(0)$ & $0.000291740127910(1)$ \\
\hline $8 D$ & $0.01104215(4)$ & $0.0109705203(3)$ & & & \\
\hline $9 D$ & $0.00775899(1)$ & $0.0077075908(4)$ & $8 K$ & $0.000370171398911(0)$ & $0.000370171398911(0)$ \\
\hline \multirow[t]{2}{*}{$10 D$} & $0.00565819(1)$ & $0.0056201391(2)$ & $9 K$ & $0.000260000663720(0)$ & $0.000260000663720(0)$ \\
\hline & & & $10 K$ & $0.000189549557(1)$ & $0.000189549559(2)$ \\
\hline $4 F$ & $0.029888962(1)$ & $0.029886876415(8)$ & & & \\
\hline $5 F$ & $0.0153396693(5)$ & $0.01533790034(1)$ & & & \\
\hline $6 F$ & $0.0088884529(2)$ & $0.008887176000(4)$ & & & \\
\hline $7 F$ & $0.00560164751(1)$ & $0.005600739943(3)$ & & & \\
\hline $8 F$ & $0.0037544983(3)$ & $0.003753842921(6)$ & & & \\
\hline $9 F$ & $0.0026377782(5)$ & $0.00263729460(1)$ & & & \\
\hline $10 F$ & $0.0019233924(5)$ & $0.001923027260(9)$ & & & \\
\hline
\end{tabular}
the Rydberg electron on the Lamb shift of the $1 s$ electron. For $L \geq 1$, the additional contribution to the energy is

TABLE XIII. Variational $Q$ matrix elements (in units of $10^{-3} \alpha^{3}$ a.u.). 


$$
\begin{aligned}
\Delta E_{L, \beta} & =-\frac{4 \alpha^{3}}{3 Z^{5}}\left\langle\delta\left(\mathbf{r}_{1}\right)+\delta\left(\mathbf{r}_{2}\right)\right\rangle 0.31626\left\langle x^{-4}\right\rangle_{n L} \\
& \rightarrow-\frac{4 \alpha^{3}}{3 \pi Z^{2}} 0.31626\left\langle x^{-4}\right\rangle_{n L} .
\end{aligned}
$$

For $L=1$, the value is taken to be $0.5 \Delta E_{L, \beta}$ with $\pm 0.5 \Delta E_{L, \beta}$ as the uncertainty. For $L>1$, the values are $\Delta E_{L, \beta}$ with $\pm 1.5 \Delta E_{L, \beta}\left\langle x^{-6}\right\rangle_{n L} /\left\langle x^{-4}\right\rangle_{n L}$ as an estimate of the contribution from higher-order terms. This substantially reduces the previous theoretical uncertainties [4], which were taken to be $4 \%$ of $\Delta E_{L, 1}$. Including this term, the full asymptotic expansion for $\Delta E_{L, 1}$ is

$$
\begin{aligned}
\Delta E_{L, 1} \rightarrow \frac{4 \alpha^{3} Z}{3 \pi}\{ & \left(Z^{3}-\frac{31}{2} Z^{-3}\left\langle x^{-4}\right\rangle_{n L}+\frac{1447}{16} Z^{-5}\left\langle x^{-6}\right\rangle_{n L}\right) \\
& \times\left[\ln (Z \alpha)^{-2}+\frac{19}{30}-\beta_{1 s}-\left[\frac{Z-1}{Z}\right]^{4} n^{-3} \beta_{n L}-0.31626 Z^{-6}\left\langle x^{-4}\right\rangle_{n L}\right. \\
& \left.\left.+2.296 \pi \alpha Z+O\left(\alpha^{2} Z^{2}\right)+y C_{M}\right]\right\}-\Delta E_{L}(1 s),
\end{aligned}
$$

\begin{tabular}{|c|c|c|c|c|}
\hline State & $b_{3,2}\left(n^{3} L_{L}\right)$ & $b_{3, Z}^{\mathrm{X}}\left(n^{3} L_{L}\right)$ & $b_{3, Z}\left(n^{3} L_{L}-n^{1} L_{L}\right)$ & $b_{3, Z}^{\mathrm{X}}\left(n^{3} L_{L}-n^{1} L_{L}\right)$ \\
\hline $\begin{array}{r}2 P \\
3 P \\
4 P \\
5 P \\
6 P \\
7 P \\
8 P \\
9 P \\
10 P\end{array}$ & $\begin{array}{l}-34.65920746(2) \\
-10.05257679(9) \\
-4.17795598(4) \\
-2.118552751(5) \\
-1.217922615(1) \\
-0.76330332(5) \\
-0.50950454(2) \\
-0.3568300582(2) \\
-0.25953925(2)\end{array}$ & $\begin{array}{c}-116.4508(3) \\
-27.60494(7) \\
-10.4555(3) \\
-5.0354(8) \\
-2.80274(7) \\
-1.7182(3) \\
-1.129(2) \\
-0.78113(6) \\
-0.5625(2)\end{array}$ & $\begin{array}{c}-17.88657999(3) \\
-5.24803680(5) \\
-2.20144916(5) \\
-1.1230906978(3) \\
-0.64833108961(3) \\
-0.40754330(3) \\
-0.27264806(1) \\
-0.191283118(3) \\
-0.13932418(4)\end{array}$ & $\begin{array}{l}9.16233(5) \\
3.71221(6) \\
1.6751(1) \\
0.8788(5) \\
0.51411(3) \\
0.3258(1) \\
0.2191(6) \\
0.154110(4) \\
0.11246(5)\end{array}$ \\
\hline $\begin{array}{r}3 D \\
4 D \\
5 D \\
6 D \\
7 D \\
8 D \\
9 D \\
10 D\end{array}$ & $\begin{array}{l}-1.253936932(3) \\
-0.53039601280(3) \\
-0.271843243(1) \\
-0.157392102(2) \\
-0.0991400595(1) \\
-0.066424942115(2) \\
-0.04665582877(1) \\
-0.034013509850(2)\end{array}$ & $\begin{array}{l}-0.568432(3) \\
-0.229403(6) \\
-0.11503305(6) \\
-0.065820(6) \\
-0.041175(2) \\
-0.0274639(7) \\
-0.0192338(5) \\
-0.0139916(2)\end{array}$ & $\begin{array}{l}-0.882271458(1) \\
-0.3726367052(2) \\
-0.1908640374(2) \\
-0.110469724(2) \\
-0.06957052334(6) \\
-0.04660735914(6) \\
-0.0327336325(2) \\
-0.0238624752(3)\end{array}$ & $\begin{array}{l}0.418403043(9) \\
0.175223(3) \\
0.089422(2) \\
0.051657(6) \\
0.032500(4) \\
0.021760(3) \\
0.0152789(1) \\
0.0111333693(1)\end{array}$ \\
\hline $\begin{array}{r}4 F \\
5 F \\
6 F \\
7 F \\
8 F \\
9 F \\
10 F\end{array}$ & $\begin{array}{l}-0.18628815635(8) \\
-0.095404200928(6) \\
-0.05521775603(1) \\
-0.034775098501(6) \\
-0.023297542106(9) \\
-0.016363016854(3) \\
-0.01192882958(3)\end{array}$ & $\begin{array}{l}-0.09380225(5) \\
-0.04808442(1) \\
-0.02784255(7) \\
-0.0175386(4) \\
-0.011751514(5) \\
-0.00825435(3) \\
-0.00601792(4)\end{array}$ & $\begin{array}{l}-0.13168144279(3) \\
-0.067429304485(3) \\
-0.03902362820(1) \\
-0.02457522810(3) \\
-0.01646366110(1) \\
-0.01156300792645(2) \\
-0.00842944380(1)\end{array}$ & $\begin{array}{l}0.065366845(7) \\
0.03342909(2) \\
0.019333992(8) \\
0.0121710(2) \\
0.0081519185(9) \\
0.005724550(6) \\
0.00417280(2)\end{array}$ \\
\hline $\begin{array}{r}5 G \\
6 G \\
7 G \\
8 G \\
9 G \\
10 G\end{array}$ & $\begin{array}{l}-0.044457362185(8) \\
-0.025728898919(3) \\
-0.016202894306(3) \\
-0.010854849186(3) \\
-0.007623785564(1) \\
-0.0055577771324(8)\end{array}$ & $\begin{array}{l}-0.022280002(1) \\
-0.01289766(2) \\
-0.00812368(3) \\
-0.005442831(1) \\
-0.0038229594(4) \\
-0.00278706452(5)\end{array}$ & $\begin{array}{l}-0.031434295887(6) \\
-0.0181915851056(9) \\
-0.011456060115(2) \\
-0.007674711800(2) \\
-0.0053902126448(2) \\
-0.0039294715568(4)\end{array}$ & $\begin{array}{l}0.015686016(7) \\
0.0090754430(3) \\
0.00571441(4) \\
0.003827879(3) \\
0.00268829775(5) \\
0.00195968930(8)\end{array}$ \\
\hline $\begin{array}{r}6 H \\
7 H \\
8 H \\
9 H \\
10 H\end{array}$ & $\begin{array}{l}-0.01403032160004 \\
-0.0088355355517(3) \\
-0.0059191648868(3) \\
-0.004157239529(1) \\
-0.0030306377769(4)\end{array}$ & $\begin{array}{l}-0.00702453377 \\
-0.004423990(6) \\
-0.002963890(9) \\
-0.002081715(3) \\
-0.0015176040(4)\end{array}$ & $\begin{array}{l}-0.00992079160146(2) \\
-0.0062475373312(4) \\
-0.0041853770739(4) \\
-0.0029395301520(8) \\
-0.0021429200688(2)\end{array}$ & $\begin{array}{l}0.004955211453(7) \\
0.0031202943(8) \\
0.002090274(6) \\
0.001468034(2) \\
0.0010701756(1)\end{array}$ \\
\hline $\begin{array}{r}7 I \\
8 I \\
9 I \\
10 I\end{array}$ & $\begin{array}{l}-0.0053398078656(1) \\
-0.0035772692842(1) \\
-0.0025124374367(1) \\
-0.0018315702756(3)\end{array}$ & $\begin{array}{l}-0.0026728163(2) \\
-0.001790628(2) \\
-0.00125763889(9) \\
-0.0009168324(1)\end{array}$ & $\begin{array}{l}-0.00377579654013(8) \\
-0.00252949442501(8) \\
-0.00177654714804(8) \\
-0.0012951039286(2)\end{array}$ & $\begin{array}{l}0.0018863676(1) \\
0.0012636943(5) \\
0.00088752090(6) \\
0.0006469981(2)\end{array}$ \\
\hline $\begin{array}{r}8 K \\
9 K \\
10 K\end{array}$ & $\begin{array}{l}-0.002325176 \quad 073824 \\
-0.00163304812996(1) \\
-0.00119049340044(2)\end{array}$ & $\begin{array}{l}-0.00116376586744 \\
-0.00081735879(3) \\
-0.0005958589(1)\end{array}$ & $\begin{array}{l}-0.001644144756049 \\
-0.00115473643790 \\
-0.00084180333198\end{array}$ & $\begin{array}{l}0.00082146678810 \\
0.00057693803850 \\
0.0004205857(1)\end{array}$ \\
\hline
\end{tabular}

TABLE XIV. Variational matrix elements $\left\langle B_{3, Z}\right\rangle=b_{3, Z}+(\mu / M) b_{3, Z}$ (in units of $10^{-3} \alpha^{2}$ a.u.). 
where the $\beta_{n L}$ are hydrogen-atom Bethe logarithms [50], the term $y C_{M}$ denotes finite-mass corrections [5,46-48], and $\Delta E_{L}(1 s)$ is the $\mathrm{He}^{+}(1 s)$ Lamb shift which is subtracted. For $L \leq 3$, the asymptotic expansion represented by first group of terms in parentheses should be replaced by the correct matrix element $\left\langle\delta\left(\mathbf{r}_{1}\right)+\delta\left(\mathbf{r}_{2}\right)\right\rangle$. For lowlying states, the corrections of relative $O\left(\alpha^{2} Z^{2}\right)$ are also important, and are included in the calculations in a oneelectron approximation as described in Ref. [5]. Equation (136) does not apply to $S$ states. In this case, a $1 / Z$ expansion is used instead for the Bethe logarithm $[5,23,51,52]$. Very accurate values for the $1{ }^{1} S$ and $2{ }^{1} S$ states have recently been calculated by Baker et al. [53].
Finally, $\Delta E_{L, 2}$ denotes the Araki-Sucher electronelectron QED energy shift given by Eq. (118). The asymptotic expansion follows simply by inserting Eq. (119) for $Q$, and neglecting the $\left\langle\delta\left(\mathbf{r}_{12}\right)\right\rangle$ terms.

The result of adding all the above contributions is summarized in Table $\mathrm{XX}$ for the states up to $n=10$ and $L=7$. A detailed listing of the individual terms in Eq. (125) is given in Ref. [5]. The $2{ }^{1} S$ and $2{ }^{3} S$ states are renormalized to the high-precision measurements of the $2{ }^{1} S_{0}-n{ }^{1} D_{2}$ [54] and $2{ }^{3} S_{1}-n{ }^{3} D_{1}$ [55] transition frequencies. The comparison with theory allows a precise determination of the $S$-state energies because the theoretical uncertainties are relatively much smaller for the $D$ states

TABLE XV. Variational matrix elements $\left\langle B_{3, e}\right\rangle=b_{3, e}+(\mu / M) b_{3, e}^{\mathrm{X}}$ (in units of $10^{-3} \alpha^{2}$ a.u.).

\begin{tabular}{|c|c|c|c|c|}
\hline State & $b_{3, e}\left(n^{3} L L\right)$ & $b_{3, e^{\mathrm{X}}}^{\left(n^{3} L_{L}\right)}$ & $b_{3, e}\left(n^{3} L_{L}-n^{1} L_{L}\right)$ & $b_{3, e^{\mathrm{X}}}^{\mathrm{X}}\left(n^{3} L_{L}-n^{1} L_{L}\right)$ \\
\hline $2 P$ & $51.47807556(4)$ & $159.91191(2)$ & $-6.460340383(6)$ & $5.34018727(2)$ \\
\hline $3 P$ & $14.6976631019(3)$ & $36.69106(1)$ & $-1.796214099(5)$ & $1.7743914(2)$ \\
\hline $4 P$ & $6.072474598(1)$ & $13.72336(4)$ & $-0.739628814(2)$ & $0.761251(5)$ \\
\hline $5 P$ & $3.07067152(7)$ & $6.5666(1)$ & $-0.3741322165(2)$ & $0.390876(9)$ \\
\hline $6 P$ & $1.76260391(4)$ & $3.64028(1)$ & $-0.2149869172(7)$ & $0.22618(1)$ \\
\hline $7 P$ & $1.10365755(2)$ & $2.2255(2)$ & $-0.134769320(4)$ & $0.142344(1)$ \\
\hline $8 P$ & $0.7362529289467(4)$ & $1.460(1)$ & $-0.090000449(6)$ & $0.09525(5)$ \\
\hline $9 P$ & $0.515422689(3)$ & $1.00835(6)$ & $-0.063065064(4)$ & $0.06687(1)$ \\
\hline $10 P$ & $0.37478201(8)$ & $0.7254(2)$ & $-0.045894449(7)$ & $0.04871(1)$ \\
\hline $3 D$ & $10.8776152411(9)$ & $1.741123(2)$ & $-0.43363792551(2)$ & $0.42532598(6)$ \\
\hline $4 D$ & $0.794033195(3)$ & $0.713162(3)$ & $-0.1821171930(1)$ & $0.178131(2)$ \\
\hline $5 D$ & $0.4069310424233(7)$ & $0.360153(6)$ & $-0.0930329550(2)$ & $0.09088716(9)$ \\
\hline $6 D$ & $0.2355954914(7)$ & $0.2068786(8)$ & $-0.0537682643(1)$ & $0.0524967(3)$ \\
\hline $7 D$ & $0.148396142(7)$ & $0.1297040(2)$ & $-0.0338319355(2)$ & $0.033021(2)$ \\
\hline $8 D$ & $0.099425529(2)$ & $0.08663(2)$ & $-0.0226520830(2)$ & $0.02210445(5)$ \\
\hline $9 D$ & $0.069834185(2)$ & $0.060728(1)$ & $-0.0159029479(3)$ & $0.01551762(3)$ \\
\hline $10 D$ & $0.050910856(1)$ & $0.044218(2)$ & $-0.0115898291(3)$ & $0.0113064757(3)$ \\
\hline $4 F$ & $0.2792502060495(4)$ & $0.27987344(3)$ & $-0.065749728329(5)$ & $0.06560496(2)$ \\
\hline $5 F$ & $0.14297629447(3)$ & $0.14328235(6)$ & $-0.033648680555(7)$ & $0.03357108(2)$ \\
\hline $6 F$ & $0.08273964627(2)$ & $0.0829081(3)$ & $-0.019467347857(3)$ & $0.01942176(4)$ \\
\hline $7 F$ & $0.05210336716(4)$ & $0.0522046(4)$ & $-0.012257182505(2)$ & $0.0122283(1)$ \\
\hline $8 F$ & $0.03490463245(2)$ & $0.034970(1)$ & $-0.00821037617(1)$ & $0.00819106(7)$ \\
\hline $9 F$ & $0.02451430091(9)$ & $0.0245589(3)$ & $-0.00576591823(3)$ & $0.0057523812(6)$ \\
\hline $10 F$ & $0.01787071395(4)$ & $0.0179029(4)$ & $-0.004203088674(8)$ & $0.00419325(3)$ \\
\hline $5 G$ & $0.06667851829769(2)$ & 0.0667402003404 & $-0.01571369225067(2)$ & 0.0157015518030 \\
\hline $6 G$ & $0.0385870858526(4)$ & $0.0386254156(9)$ & $-0.00909291431222(2)$ & $0.009085836(1)$ \\
\hline $7 G$ & $0.0242996468156(4)$ & $0.02432463(3)$ & $-0.0057258714362(2)$ & $0.005721425(5)$ \\
\hline $8 G$ & $0.016278795107(1)$ & $0.016295878(6)$ & $-0.00383575593503(3)$ & $0.0038327959(6)$ \\
\hline $9 G$ & $0.0114330722451(1)$ & $0.011445238(2)$ & $-0.00269390745148(1)$ & $0.0026918401(3)$ \\
\hline $10 G$ & $0.008334680076(2)$ & $0.008343593385(1)$ & $-0.0019638222144(1)$ & $0.0019623258(1)$ \\
\hline $6 H$ & 0.0021044897027262 & 0.0210566524671 & -0.004960127672240 & 0.0049575564513 \\
\hline $7 H$ & $0.01325277394391(1)$ & $0.01326043263(3)$ & -0.003123526153029 & 0.0031219003041 \\
\hline $8 H$ & $0.00887832009777(4)$ & $0.0088835514(3)$ & $-0.00209249280742(1)$ & 0.0020914042489 \\
\hline $9 H$ & $0.0062355234242(9)$ & $0.0062392386(5)$ & -0.001469611196255 & $0.0014688482(1)$ \\
\hline $10 H$ & $0.004545692830407(2)$ & $0.0045484226(2)$ & $-0.00107133915486(2)$ & $0.001070784242(9)$ \\
\hline $7 I$ & $0.00800963994531(1)$ & $0.0080133525(1)$ & -0.001887865368769 & 0.00188701519656 \\
\hline $8 I$ & 0.005365835473893 & 0.00536835725704 & -0.001264715866287 & 0.00126414513318 \\
\hline $9 I$ & $0.00376859792847(1)$ & $0.00377038430(5)$ & $-0.00088824690951(0)$ & $0.000887845947(5)$ \\
\hline $10 I$ & $0.002747307540223(3)$ & $0.00274861703(2)$ & -0.000647530040294 & $0.00064723786(1)$ \\
\hline $8 K$ & 0.003487751991873 & 0.00348926170351 & -0.000822066829645 & 0.00082171526394 \\
\hline $9 K$ & 0.002449560245702 & 0.00245062670542 & -0.000577362748041 & 0.00057711556821 \\
\hline $10 K$ & 0.001785729532702 & 0.00178651002563 & -0.000420896827379 & $0.000420716574(8)$ \\
\hline
\end{tabular}


TABLE XVI. Variational matrix elements $\left\langle B_{5}\right\rangle=b_{5}+(\mu / M) b_{5}^{\mathrm{X}}$ (in units of $10^{-3} \alpha^{2}$ a.u.).

\begin{tabular}{|c|c|c|c|c|c|}
\hline State & $b_{5}\left(n^{3} L_{L}\right)$ & $b_{5}^{\mathrm{x}}\left(n^{3} L_{L}\right)$ & State & $b_{5}\left(n^{3} L_{L}\right)$ & $b_{5}^{\mathrm{X}}\left(n^{3} L_{L}\right)$ \\
\hline $2 P$ & $-22.52016585(1)$ & $-54.06886(1)$ & $5 G$ & $-0.044444887727817(5)$ & $-0.0000418828(2)$ \\
\hline $3 P$ & $-6.157504600(1)$ & $-11.639505(4)$ & $6 G$ & $-0.025718502596952(6)$ & $-0.000026329(2)$ \\
\hline $4 P$ & $-2.5092699346(1)$ & $-4.23654(1)$ & $7 G$ & $-0.0161950948554(2)$ & $-0.00001731(2)$ \\
\hline $5 P$ & $-1.26111206(2)$ & $-1.99616(4)$ & $8 G$ & $-0.0108490671536(8)$ & $-0.0000118819(1)$ \\
\hline $6 P$ & $-0.72153649(1)$ & $-1.095507(7)$ & $9 G$ & $-0.007619452487(2)$ & $-0.000008476(2)$ \\
\hline $7 P$ & $-0.450914432(5)$ & $-0.66498(6)$ & $10 G$ & $-0.0055544749552(9)$ & $-0.0000062355327(6)$ \\
\hline $8 P$ & $-0.30043027313(4)$ & $-0.4339(4)$ & & & \\
\hline $9 P$ & $-0.2101406073(6)$ & $-0.29845(2)$ & $6 H$ & -0.014029355809429 & -0.000007850884091 \\
\hline $10 P$ & $-0.15270790(2)$ & $-0.21404(7)$ & $7 H$ & $-0.00883466183184(1)$ & $-0.0000051227(6)$ \\
\hline $3 D$ & $-1.2296097200(2)$ & $-0.005851(2)$ & $\begin{array}{l}8 H \\
9 H\end{array}$ & $\begin{array}{l}-0.00591845962755(3) \\
-0.0041566850209(2)\end{array}$ & $\begin{array}{l}-0.00000350203(3) \\
-0.0000024902(1)\end{array}$ \\
\hline $4 D$ & $-0.516865792(2)$ & $-0.00227872(2)$ & $10 H$ & $-0.003030202159079(1)$ & -0.000001830371386 \\
\hline $5 D$ & $-0.2641406639(1)$ & $-0.001278(4)$ & & & \\
\hline $6 D$ & $-0.1526909831(4)$ & $-0.000809(1)$ & $7 I$ & -0.005339689422330 & -0.000002475412823 \\
\hline $7 D$ & $-0.096087155(2)$ & $-0.0005465(4)$ & $8 I$ & -0.003577156428906 & -0.000001681769463 \\
\hline $8 D$ & $-0.0643395868(4)$ & $-0.0003825(7)$ & $9 I$ & -0.002512341431804 & -0.000001191548688 \\
\hline $9 D$ & $-0.0451719059(6)$ & $-0.000280(2)$ & $10 I$ & $-0.001831491336303(3)$ & -0.000000865984456 \\
\hline \multirow[t]{2}{*}{$10 D$} & $-0.0329216729(4)$ & $-0.000213(2)$ & & & \\
\hline & & & $8 K$ & -0.002325156103409 & -0.000001002917144 \\
\hline $4 F$ & $-0.185960647706(7)$ & $-0.00058426(2)$ & $9 K$ & -0.001633028437480 & -0.000000711005402 \\
\hline $5 F$ & $-0.09516693347(2)$ & $-0.0003438(2)$ & $10 K$ & -0.001190475983466 & -0.000000517636417 \\
\hline $6 F$ & $-0.055057905123(9)$ & $-0.00021220(3)$ & & & \\
\hline $7 F$ & $-0.03466573139(2)$ & $-0.0001384(7)$ & & & \\
\hline $8 F$ & $-0.02322045222(4)$ & $-0.0000946(6)$ & & & \\
\hline $9 F$ & $-0.01630702132(9)$ & $0.00006717(7)$ & & & \\
\hline $10 F$ & $-0.01188703832(3)$ & $-0.0000496(2)$ & & & \\
\hline
\end{tabular}

TABLE XVII. Variational matrix elements $\left\langle\Delta_{3}\right\rangle$ (in units of $10^{-3} \alpha^{2} \mu / M$ a.u.).

\begin{tabular}{|c|c|c|c|c|c|}
\hline State & $\Delta_{3}\left(n^{3} L_{L}\right)$ & $\Delta_{3}\left(n^{3} L_{L}-n^{1} L_{L}\right)$ & State & $\Delta_{3}\left(n^{3} L_{L}\right)$ & $\Delta_{3}\left(n^{3} L_{L}-n^{1} L_{L}\right)$ \\
\hline $2 P$ & $99.554499868(2)$ & $-12.7442104(1)$ & $5 G$ & $0.0444594340(1)$ & $-0.03142738825(1)$ \\
\hline $3 P$ & $29.31995723(8)$ & $-3.5758186(3)$ & $6 G$ & $0.025730566(2)$ & $-0.018185828(3)$ \\
\hline $4 P$ & $12.213608(1)$ & $-1.4762738(4)$ & $7 G$ & $0.01620413(2)$ & $-0.01145176(2)$ \\
\hline $5 P$ & $6.1971768(3)$ & $-0.7474830(4)$ & $8 G$ & $0.010855763(1)$ & $-0.007671516(1)$ \\
\hline $6 P$ & $3.56351401(1)$ & $-0.4297127(1)$ & $9 G$ & $0.007624465(3)$ & $-0.005387820(7)$ \\
\hline $7 P$ & $2.233594(2)$ & $-0.2694353(6)$ & $10 G$ & $0.00555829501(7)$ & $-0.0039276487(9)$ \\
\hline $8 P$ & $1.491005(1)$ & $-0.1799521(9)$ & & & \\
\hline $9 P$ & $1.04426122(2)$ & $-0.12610752(4)$ & $6 H$ & $0.0140304989(1)$ & $-0.00992025578(2)$ \\
\hline \multirow[t]{2}{*}{$10 P$} & $0.7595575(6)$ & $-0.09177703(8)$ & $7 H$ & $0.0088356936(1)$ & $-0.00624705227(3)$ \\
\hline & & & $8 H$ & $0.0059192911(1)$ & $-0.00418498487(1)$ \\
\hline $3 D$ & $1.2027456(1)$ & $-0.867276123(1)$ & $9 H$ & $0.004157344(4)$ & $-0.002939226(3)$ \\
\hline $4 D$ & $0.5003921(1)$ & $-0.36423456(1)$ & $10 H$ & $0.003030717(4)$ & $-0.002142679(3)$ \\
\hline $5 D$ & $0.2544323797(7)$ & $-0.186065886(5)$ & & & \\
\hline $6 D$ & $0.146663787(5)$ & $-0.1075359(2)$ & $7 I$ & $0.0053398301(2)$ & $-0.00377573082(2)$ \\
\hline $7 D$ & $0.09213578(3)$ & $-0.06766366(5)$ & $8 I$ & $0.003577290415(2)$ & $-0.002529431806(1)$ \\
\hline $8 D$ & $0.0616242(4)$ & $-0.0453043(2)$ & $9 I$ & $0.002512455080(5)$ & $-0.001776493658(4)$ \\
\hline $9 D$ & $0.0432319(1)$ & $-0.03180612(3)$ & $10 I$ & $0.0018315854(3)$ & $-0.0012950605(1)$ \\
\hline \multirow[t]{2}{*}{$10 D$} & $0.03149027(2)$ & $-0.02317976(2)$ & & & \\
\hline & & & $8 K$ & $0.00232517985(1)$ & $-0.001644133661(9)$ \\
\hline $4 F$ & $0.186290136(2)$ & $-0.131499464(2)$ & $9 K$ & $0.001633051862(1)$ & $-0.001154725509(1)$ \\
\hline $5 F$ & $0.09539944(2)$ & $-0.06729736(2)$ & $10 K$ & $0.00119049648(3)$ & $-0.00084179351(2)$ \\
\hline $6 F$ & $0.05521261(4)$ & $-0.03893470(2)$ & & & \\
\hline $7 F$ & $0.03477077(2)$ & $-0.02451434(2)$ & & & \\
\hline $8 F$ & $0.02329413(2)$ & $-0.0164207181(6)$ & & & \\
\hline $9 F$ & $0.016360371(7)$ & $-0.01153180893(2)$ & & & \\
\hline $10 F$ & $0.011926768(8)$ & $-0.00840616(2)$ & & & \\
\hline
\end{tabular}


TABLE XVIII. Convergence of the spin-dependent matrix elements $\left\langle 2^{3} P_{1}\left|B_{i}\right|{ }^{3} P_{1}\right\rangle$ for helium, and comparison with previous calculations (in units of $10^{-3} \alpha^{2}$ a.u.).

\begin{tabular}{|c|c|c|c|}
\hline$N^{\mathrm{a}}$ & $\left\langle B_{3, Z}\right\rangle$ & $\left\langle B_{3, e}\right\rangle$ & $\left\langle B_{5}\right\rangle$ \\
\hline 104 & -34.659788995 & 51.477882212 & -2.2520333177 \\
\hline 145 & -34.659291413 & 51.477935509 & -2.2520188358 \\
\hline 197 & -34.659222961 & 51.478041652 & -2.2520168778 \\
\hline 264 & -34.659214323 & 51.478116882 & -2.2520180584 \\
\hline 342 & -34.659209437 & 51.478086912 & -2.2520169793 \\
\hline 436 & -34.659209239 & 51.478080805 & -2.2520167695 \\
\hline 539 & -34.659207952 & 51.478076407 & -2.2520166156 \\
\hline 658 & -34.659207782 & 51.478075998 & -2.2520166007 \\
\hline 724 & -34.659207542 & 51.478075713 & -2.2520165905 \\
\hline 804 & -34.659207475 & 51.478075603 & -2.2520165868 \\
\hline Extrapolation & $-34.659207456(19)$ & $51.478075561(42)$ & $-2.2520165855(13)$ \\
\hline $\begin{array}{l}\text { Lewis and } \\
\text { Serafino }^{b}\end{array}$ & -34.659107 & 51.476434 & -2.251977 \\
\hline$\left\langle B_{3, Z}\right\rangle+\left\langle B_{3, e}\right\rangle$ & \multicolumn{2}{|c|}{$16.818868105(46)$} & \\
\hline Schwartz $^{\mathrm{c}}$ & \multicolumn{2}{|c|}{$16.818822(5)$} & $-2.2520163(5)$ \\
\hline
\end{tabular}

${ }^{a} N$ is the number of terms in the doubled basis sets corresponding to $\Omega=4,5, \ldots, 13$ in Table 2 of Ref. $[5]$.

${ }^{\mathrm{b}}$ Reference [31].

${ }^{\mathrm{c}}$ Reference [43].

$( \pm 100 \mathrm{kHz}$ or less, see the table).

Table XXI compares the spin-averaged energy shifts calculated variationally with those obtained entirely from the asymptotic expansions described above. This short table is of key importance because it establishes the degree of convergence of the asymptotic expansions, in addition to verifying the correctness of the much more elaborate variational matrix elements. The agreement to within $100 \mathrm{~Hz}$ for the $K$ states indicates that the asymptotic expansions are substantially more accurate than $\pm 100 \mathrm{~Hz}$ for the $L$ and $M$ states. For this reason, the variational calculations have not been pursued beyond the $K$ states.

With the above results in hand, one can confidently take the asymptotic expansions as correct to better than $\pm 100 \mathrm{~Hz}$ for the $L$ and $M$ states. The detailed results for the various contributions are presented in Table XXII in

TABLE XIX. Values of $R_{M} / n^{2}$ (in MHz) for ${ }^{4} \mathrm{He}$.

\begin{tabular}{rc}
\hline$n$ & $R_{M} / n^{2}$ \\
\hline 1 & $3289391007.44(54)$ \\
2 & $822347751.86(13)$ \\
3 & $365487889.716(60)$ \\
4 & $205586937.965(34)$ \\
5 & $131575640.298(22)$ \\
6 & $91371972.429(15)$ \\
7 & $67130428.723(11)$ \\
8 & $51396734.4913(84)$ \\
9 & $40609765.5240(67)$ \\
10 & $32893910.0744(54)$ \\
11 & $27185049.6483(45)$ \\
12 & $22842993.1072(37)$ \\
\hline \hline
\end{tabular}

the same format as used previously for the variational calculations [5]. Note that the relatively large uncertainty from $\Delta E_{s t}$ should not be included in the spin-averaged energy because it cancels on taking the average. The other uncertainties are common to all four components and so should only be included once in the spin average. It is useful to remember that, with the exception of $\Delta E_{s t}$, the singlet value for each quantity equals the spin-averaged value in the asymptotic limit.

Except for the higher-lying $S$ states, Table XXII completes the tabulation of energies for all states of helium up to $n=10$. A paper on the $S$ states is in preparation.

\section{COMPARISON WITH EXPERIMENT}

Reference [5] presents a detailed discussion of recent high-precision experiments, which will not be repeated here. In summary, measured singlet and triplet transition frequencies of the type $2 S-2 P, 2 S-3 P$, and $2 S-n D$ are in generally satisfactory agreement with theory at the \pm 2 $\mathrm{MHz}$ level of accuracy or better when the $2 S$ states are renormalized to the values shown in Table XX. For the $2{ }^{3} S_{1}$ state, the renormalization is $12.70 \pm 2.4 \mathrm{MHz}$, and, using the new Bethe logarithm of Baker et al. [53], the renormalization for the $2{ }^{1} S_{0}$ state is only $0.75 \pm 0.15$ MHz. The agreement is as good as can be expected, and further theoretical progress will require a complete evaluation of the $O\left(\alpha^{4}\right)$ QED contributions.

The one experiment requiring further discussion is the work of Hessels et al. [1] on the transition frequencies among the $n=10$ states of helium. As shown in Table XXIII, the differences between theory (including the $\Delta V_{\text {ret }}^{\prime \prime}$ retardation correction) and experiment remain substantially larger than the $\Delta V_{\text {ret }}^{\prime \prime}$ term listed separately in the table. Unfortunately, the new result for the $10 L$ ener- 
gy in Table XXI does not give a predicted $10 K-10 L$ transition frequency which fits the previous pattern of monotonically decreasing differences from experiment (although the experimental uncertainty is larger in this case). Since the theoretical uncertainties have now been markedly reduced with the evaluation of the $\Delta E_{L, \beta}$ term in Eq. (136), there does not appear to be a ready explanation for the differences. As a representative example of higher-order terms, the last column of Table XXIII lists contributions from the spin-averaged Dirac energies of order $\alpha^{4}(Z-1)^{6}$ given by

$$
\begin{aligned}
\Delta E_{D}^{(4)}=-\alpha^{4}(Z-1)^{6} & \left(\frac{L^{-2}+(L+1)^{-2}}{8 n^{3}(2 L+1)}+\frac{3}{8 n^{4} L(L+1)}\right. \\
& \left.-\frac{3}{4 n^{5}\left(L+\frac{1}{2}\right)}+\frac{5}{16 n^{6}}\right)
\end{aligned}
$$

TABLE XX. Total calculated energies of helium, relative to ${ }^{4} \mathrm{He}^{+}(1 s)$, in units of MHz. The quantity $R_{M} / n^{2}$ from Table XIX

\begin{tabular}{|c|c|c|c|c|}
\hline State & $E\left(n^{1} L_{L}\right)$ & $E\left(n^{3} L_{L-1}\right)$ & $E\left(n^{3} L_{L}\right)$ & $E\left(n^{3} L_{L+1}\right)$ \\
\hline $2 S$ & $-137984289.00(15)^{a}$ & & $-330494992.7(2.4)^{\mathrm{a}}$ & \\
\hline $\begin{array}{r}2 P \\
3 P \\
4 P \\
5 P \\
6 P \\
7 P \\
8 P \\
9 P \\
10 P\end{array}$ & $\begin{array}{c}7638599.5(1.8) \\
2699920.4(6) \\
1189726.7(3) \\
620098.18(13) \\
362161.73(8) \\
229301.10(5) \\
154147.04(3) \\
108519.09(2) \\
79244.730(17)\end{array}$ & $\begin{array}{c}-53730894.1(1.8) \\
-16622013.8(6) \\
-7071103.4(3) \\
-3627803.20(13) \\
-2100069.37(8) \\
-1322158.07(5) \\
-885357.65(3) \\
-621517.79(2) \\
-452874.336(17)\end{array}$ & $\begin{array}{c}-53760517.5(1.8) \\
-16630129.1(6) \\
-7074411.0(3) \\
-3629465.46(13) \\
-2101020.39(8) \\
-1322752.38(5) \\
-885753.61(3) \\
-621794.75(2) \\
-453075.597(17)\end{array}$ & $\begin{array}{c}-53762811.0(1.8) \\
-16630788.5(6) \\
-7074680.8(3) \\
-3629601.02(13) \\
-2101097.90(8) \\
-1322800.79(5) \\
-885785.85(3) \\
-621817.29(2) \\
-453091.976(17)\end{array}$ \\
\hline $\begin{array}{r}3 D \\
4 D \\
5 D \\
6 D \\
7 D \\
8 D \\
9 D \\
10 D\end{array}$ & $\begin{array}{l}-429859.386(17) \\
-196997.901(10) \\
-104571.673(6) \\
-61683.435(3) \\
-39288.450(2) \\
-26513.6627(15) \\
-18714.7529(11) \\
-13691.8482(17)\end{array}$ & $\begin{array}{l}-531003.220(17) \\
-255609.936(10) \\
-138403.635(6) \\
-82468.176(3) \\
-52835.868(2) \\
-35788.7583(16) \\
-25324.9259(11) \\
-18560.6844(12)\end{array}$ & $\begin{array}{l}-532328.336(17) \\
-256165.167(10) \\
-138687.195(6) \\
-82632.057(3) \\
-52938.988(2) \\
-35857.8034(16) \\
-25373.4002(11) \\
-18596.0123(12)\end{array}$ & $\begin{array}{l}-532403.665(17) \\
-256201.188(10) \\
-138706.416(6) \\
-82643.402(3) \\
-52946.212(2) \\
-35862.6773(16) \\
-25376.8393(11) \\
-18598.5276(12)\end{array}$ \\
\hline $\begin{array}{r}4 F \\
5 F \\
6 F \\
7 F \\
8 F \\
9 F \\
10 F\end{array}$ & $\begin{array}{l}-33859.2264(7) \\
-19401.2326(5) \\
-11879.6226(3) \\
-7729.8487(3) \\
-5287.14915(19) \\
-3765.99397(14) \\
-2773.0292(4)\end{array}$ & $\begin{array}{l}-34091.6835(7) \\
-19554.9663(6) \\
-11981.8924(3) \\
-7799.8500(2) \\
-5336.6302(2) \\
-3802.04009(12) \\
-2799.9996(4)\end{array}$ & $\begin{array}{l}-34564.1001(7) \\
-19779.4724(6) \\
-12106.4912(3) \\
-7876.3572(2) \\
-5387.0544(2) \\
-3837.06279(12) \\
-2825.3297(4)\end{array}$ & $\begin{array}{l}-34350.0558(7) \\
-19687.1854(6) \\
-12058.3852(3) \\
-7848.0113(2) \\
-5368.8903(2) \\
-3824.69534(12) \\
-2816.5142(4)\end{array}$ \\
\hline $\begin{array}{r}5 G \\
6 G \\
7 G \\
8 G \\
9 G \\
10 G\end{array}$ & $\begin{array}{c}-4679.86428(16) \\
-3025.55240(16) \\
-2027.2392(2) \\
-1411.75810(8) \\
-1017.6837(1) \\
-755.70381(4)\end{array}$ & $\begin{array}{c}-4730.67794(17) \\
-3055.00908(16) \\
-2045.8133(3) \\
-1424.2130(1) \\
-1026.43733(7) \\
-762.08858(4)\end{array}$ & $\begin{array}{l}-4889.25025(17) \\
-3146.72746(16) \\
-2103.5488(3) \\
-1462.8801(1) \\
-1053.58852(7) \\
-781.87856(4)\end{array}$ & $\begin{array}{c}-4806.19206(17) \\
-3098.70510(16) \\
-2073.3286(3) \\
-1442.6452(1) \\
-1039.38247(7) \\
-771.52536(4)\end{array}$ \\
\hline $\begin{array}{r}6 H \\
7 H \\
8 H \\
9 H \\
10 H\end{array}$ & $\begin{array}{l}-968.2036(2) \\
-680.30688(4) \\
-487.09945(5) \\
-357.5210(2) \\
-268.84254(5)\end{array}$ & $\begin{array}{l}-989.4049(1) \\
-693.65838(4) \\
-496.04404(6) \\
-363.8031(1) \\
-273.42227(5)\end{array}$ & $\begin{array}{c}-1049.1216(1) \\
-731.26392(4) \\
-521.23663(6) \\
-381.4966(1) \\
-286.32072(5)\end{array}$ & $\begin{array}{c}-1017.8181(1) \\
-711.55082(4) \\
-508.03038(6) \\
-372.2214(1) \\
-279.55915(5)\end{array}$ \\
\hline $\begin{array}{r}7 I \\
8 I \\
9 I \\
10 I\end{array}$ & $\begin{array}{l}-263.77161(2) \\
-197.46324(2) \\
-149.00183(3) \\
-114.17395(1)\end{array}$ & $\begin{array}{l}-273.78111(2) \\
-204.16886(2) \\
-153.71142(2) \\
-117.60725(1)\end{array}$ & $\begin{array}{l}-300.20662(2) \\
-221.87184(2) \\
-166.14476(2) \\
-126.67114(1)\end{array}$ & $\begin{array}{l}-286.38000(2) \\
-212.60907(2) \\
-159.63921(2) \\
-121.92859(1)\end{array}$ \\
\hline $\begin{array}{r}8 K \\
9 K \\
10 K\end{array}$ & $\begin{array}{l}-88.25476(1) \\
-69.58260(1) \\
-54.85990(1)\end{array}$ & $\begin{array}{l}-93.44969(1) \\
-73.23118(1) \\
-57.51972(1)\end{array}$ & $\begin{array}{r}-106.57306(1) \\
-82.44813(1) \\
-64.23887(1)\end{array}$ & $\begin{array}{l}-99.72348(1) \\
-77.63745(1) \\
-60.73188(1)\end{array}$ \\
\hline
\end{tabular}
must be subtracted from the entries.

${ }^{\text {a }}$ Renormalized to the $2 S-n D$ transition frequencies (see text). 
TABLE XXI. Comparison of the variational and asymptotic results for the total spin-averaged energy shifts of helium (in units of $\mathrm{MHz})$. The $\left(\delta E_{\mathrm{RR}}\right)_{\mathrm{X}}$ term in Eq. (133) is included in the asymptotic values.

\begin{tabular}{rrrl}
\hline \hline State & Variational & Asymptotic & Difference \\
\hline $6 H$ & $-1007.4286(2)$ & $-1007.440(2)$ & $0.001(2)$ \\
$7 H$ & $-705.00829(4)$ & $-705.013(7)$ & $0.005(7)$ \\
$8 H$ & $-503.64746(5)$ & $-503.651(8)$ & $0.004(8)$ \\
$9 H$ & $-369.1432(2)$ & $-369.146(7)$ & $0.003(7)$ \\
$10 H$ & $-277.31512(5)$ & $-277.318(6)$ & $0.003(6)$ \\
$7 I$ & $-281.51941(2)$ & $-281.5195(3)$ & $0.0001(3)$ \\
$8 I$ & $-209.35287(2)$ & $-209.3530(2)$ & $0.0001(2)$ \\
$9 I$ & $-157.35230(3)$ & $-157.3524(2)$ & $0.0001(2)$ \\
$10 I$ & $-120.26144(1)$ & $-120.2616(2)$ & $0.0001(2)$ \\
$8 K$ & $-97.20937(1)$ & $-97.20939(6)$ & $0.00002(6)$ \\
$9 K$ & $-75.87172(1)$ & $-75.87173(5)$ & $0.00001(5)$ \\
$10 K$ & $-59.44466(1)$ & $-59.44467(4)$ & $0.00001(4)$ \\
$9 L$ & & $-39.72287(1)$ & \\
$10 L$ & & $-32.26875(1)$ & \\
$10 M$ & & $-18.648443(3)$ & \\
\hline \hline
\end{tabular}

The contributions are 2 orders of magnitude too small and of the wrong sign to account for the differences. Although a fully screened nuclear charge is used in the above, the corresponding terms of order $\alpha^{2}(Z-1)^{4}$ given by Eq. (63) are an excellent approximation to the correct $\Delta E_{\text {rel }}$.

It is perhaps worth while to enquire what additional energy terms of the form $\left\langle x^{-j}\right\rangle_{n L}$ might be arbitrarily added to account for the discrepancies. Values of $j<3$ do not produce corrections which decrease fast enough with $L$, and values of $j>3$ produce intolerably large corrections for the low-lying states. For example, a term of the form $2.1 \alpha^{3}\left\langle x^{-4}\right\rangle_{n L}$ a.u. would account for the discrepancies (except for the $D-F$ and $K-L$ transitions), but it also shifts the $2 P$ and $3 P$ states by 256 and $84 \mathrm{MHz}$, respectively. Although the polarization picture is of low accuracy for $P$ states, at least the order of magnitude should be correct to within a factor of 2 . For the case $j=3$, a term of the form $\alpha^{3}\left\langle x^{-3}\right\rangle_{n L} /(2 \pi)$ is a possible candidate, but even this would produce shifts of 17 and 5 $\mathrm{MHz}$, respectively, for the above $P$ states. Such shifts would clearly disrupt the existing agreement between theory and experiment for the $P$ states at the $\pm 2 \mathrm{MHz}$ level, and it seems unlikely that higher-order QED terms would be large enough to compensate. For example, Eq. (138) with a screened nuclear charge is only $\Delta E_{D}^{(4)} \simeq-0.14 \mathrm{MHz}$ for the $2 P$ state. In addition, a new contribution of the asymptotic form $\alpha^{3}\left\langle x^{-3}\right\rangle_{n L}$ would imply that the Araki-Sucher terms are incomplete, and a major readjustment of theory would be required.

\section{APPLICATION TO QUANTUM-DEFECT ANALYSIS}

The $1 / n^{2}$ terms contained in the asymptotic expansions (14) and (93) for $\varepsilon_{M}^{(2)}$ and $\left\langle B_{1}^{\mathrm{X}}+B_{4}^{\mathrm{X}}\right\rangle$ have impor- tant implications for the quantum-defect method widely used in the analysis of experimental data and extrapolations to the series limit [56]. In the quantum-defect method, the term energies for a Rydberg series of states is written in the form

$$
T_{n}=-R_{M}(Z-1)^{2} / n^{* 2}
$$

where $n^{*}=n-\delta\left(n^{*}\right)$, and the quantum defect $\delta\left(n^{*}\right)$ is a slowly-varying function of $n^{*}$ often expressed in the Ritz form

$$
\delta=\delta_{0}+\delta_{2} /(n-\delta)^{2}+\delta_{4} /(n-\delta)^{4}+\cdots
$$

(see Drake and Swainson [57] for a recent discussion). The significant point is that the above functional form is valid for the fixed experimental value of $R_{M}$ only if the leading term $-R_{M}(Z-1)^{2} / n^{2}$ fully accounts for the $1 / n^{2}$ dependence of the $T_{n}$. This will only be true if the higher-order terms in $1 / n^{2}$ are subtracted from $T_{n}$; i.e., $T_{n}$ should be replaced by $T_{n}-\delta T_{n}$ where, from Eqs. (14) and (93),

$$
\delta T_{n}=-R_{M}(Z-1)^{2} y^{2}\left(1+\frac{5}{6} \alpha^{2} Z^{2}\right) / n^{* 2} .
$$

Equivalently, if the quantum defect $\delta$ is small, one could define an effective Rydberg to be

$$
\widetilde{R}_{M}=R_{M}\left[1+y^{2}+\frac{5}{6}(y \alpha Z)^{2}\right] \text {. }
$$

For ${ }^{4} \mathrm{He}$, the correction factor is $1+1.87927 \times 10^{-8}$, which is certainly significant at current levels of experimental precision of one or two parts in $10^{10}[54,58,59]$. Without this adjustment, a quantum-defect fit may still appear to be adequate, but the higher-order terms in Eq. (140) will be abnormally large and loose their physical significance.

The physical significance of the $1+y^{2}$ correction is that the nucleus and inner electron can be thought of as a single composite particle with mass $M+m$. This increases the effective reduced mass for the Rydberg electron, and hence produces deeper binding. Note that for the variational calculations presented here, the coefficient $\frac{5}{6}$ in Eq. (142) should be replaced by $\frac{1}{2}$ as explained in connection with Eq. (116). Quantum-defect analyses of the total energies will be presented in a future publication [59].

\section{SUMMARY AND CONCLUSIONS}

The results presented here complete the tabulation of nonrelativistic energies, and lowest-order relativistic and QED corrections for all states of helium up to $n=10$, with the exception of the higher-lying $S$ states. The precision that has been achieved makes the helium spectrum up to $n=10$ as well understood as hydrogen for all practical purposes, at least in the nonrelativistic limit. As a consequence, helium now becomes a candidate for fundamental studies of higher-order QED effects in the same sense as hydrogen is, since the lowest-order terms can now be reliably subtracted from experimental data. The results for the $2^{3} \mathrm{P}$ states are of special significance because of the possibility of determining $\alpha$ from the fine- 
structure splittings. Further work is in progress to determine the higher-order corrections to a similarly improved accuracy.

The comparison with the extended asymptotic expansions for the nonrelativistic and relativistic energies summarized in Table XXI clearly establishes the equivalence of the SAT and LRI pictures defined by Hessels et al. [1] in the limit of high $L$, provided that the $\Delta V_{\text {ret }}^{\prime \prime}$ and $\Delta E_{L, 1}$ terms are treated consistently in both pictures. For lower $L$, the differences are due entirely to the lack of convergence of the asymptotic expansions, rather than to a difference in physical content. The comparison also resolves questions raised [34] concerning the adequacy of the Breit operators used in SAT relative to those of LRI. With the addition of $\Delta V_{\text {ret }}^{\prime \prime}$ to SAT, both are equally justified (or unjustified) in the high- $L$ limit.

The other significant conclusion from Table XXI is that variational calculations need not be extended beyond $L=7$ because the asymptotic expansions provide more than sufficient precision for current levels of experimental accuracy. The detailed asymptotic results for $L$ and $M$ states are those listed in Table XXII.

Finally, the interpretation of the experimental results for transition frequencies among the $n=10$ in Table XXIII remains puzzling. The present result for the $10 L$ state gives a predicted $K-L$ transition frequency which appears to fall outside the pattern of deviations shown by the previous ones. In addition, it is difficult to arbitrarily add a new $\left\langle x^{-j}\right\rangle$ term to account for the discrepancies without disrupting the existing agreement between theory and experiment for the lower-lying states. Even the case $j=3$ leads to an implausibly large shift for the $2 S-2 P$ transitions. Perhaps future experiments on Rydberg states, especially a remeasurement of the least accurately

TABLE XXII. Contributions to the energies of ${ }^{4} \mathrm{He}$, relative to $\mathrm{He}^{+}(1 s)$ in $\mathrm{MHz}$.

\begin{tabular}{|c|c|c|c|c|}
\hline Term & $9^{1} L_{8}$ & $9^{3} L_{7}$ & $9^{3} L_{8}$ & $9{ }^{3} L_{9}$ \\
\hline $\begin{array}{l}\Delta E_{\mathrm{nr}} \\
\Delta E_{\mathrm{M}}(1) \\
\Delta E_{\mathrm{M}}(2) \\
\Delta E_{\mathrm{rel}} \\
\Delta E_{\mathrm{anom}} \\
\Delta E_{\mathrm{st}} \\
\left(\Delta E_{\mathrm{RR}}\right)_{\mathrm{M}} \\
\left(\Delta E_{\mathrm{RR}}\right) \mathrm{X} \\
\Delta E_{\mathrm{nuc}} \\
\Delta E_{\mathrm{L}, 1} \\
\Delta E_{\mathrm{L}, 2} \\
\text { Total }\end{array}$ & $\begin{array}{c}-30.712304 \\
-0.008414 \\
-0.763037 \\
-8.235266(9) \\
0.000000 \\
4.90683(2) \\
-0.005219 \\
0.004225 \\
-0.000001 \\
-0.000722(3) \\
-0.002128 \\
-34.81603(2)\end{array}$ & $\begin{array}{c}-30.712304 \\
-0.008414 \\
-0.763037 \\
-6.232928(9) \\
0.000547 \\
0.000000 \\
-0.006252 \\
0.004709 \\
-0.000001 \\
-0.000722(3) \\
-0.002128 \\
-37.72053(1)\end{array}$ & $\begin{array}{c}-30.712304 \\
-0.008414 \\
-0.763037 \\
-8.431574(9) \\
-0.000911 \\
-4.90683(2) \\
-0.005219 \\
0.004279 \\
-0.000001 \\
-0.000722(3) \\
-0.002128 \\
-44.82687(2)\end{array}$ & $\begin{array}{c}-30.712304 \\
-0.008414 \\
-0.763037 \\
-9.640416(9) \\
0.000384 \\
0.000000 \\
-0.004403 \\
0.003795 \\
-0.000001 \\
-0.000722(3) \\
-0.002128 \\
-41.12725(1)\end{array}$ \\
\hline Term & $10^{1} L_{8}$ & $10{ }^{3} L_{7}$ & $10^{3} L_{8}$ & $10{ }^{3} L_{9}$ \\
\hline $\begin{array}{l}\Delta E_{\mathrm{nr}} \\
\Delta E_{\mathrm{M}}(1) \\
\Delta E_{\mathrm{M}}(2) \\
\Delta E_{\mathrm{rel}} \\
\Delta E_{\mathrm{anom}} \\
\Delta E_{\mathrm{st}} \\
\left(\Delta E_{\mathrm{RR}}\right)_{\mathrm{M}} \\
\left(\Delta E_{\mathrm{RR}}\right) \mathrm{X} \\
\Delta E_{\mathrm{nuc}} \\
\Delta E_{\mathrm{L}, 1} \\
\Delta E_{\mathrm{L}, 2} \\
\text { Total }\end{array}$ & $\begin{array}{c}-24.178633 \\
-0.006623 \\
-0.618060 \\
-7.462659(8) \\
0.000000 \\
3.57708(1) \\
-0.004271 \\
0.003607 \\
0.000000 \\
-0.000557(3) \\
-0.001551 \\
-28.69167(2)\end{array}$ & $\begin{array}{c}-24.178633 \\
-0.006623 \\
-0.618060 \\
-6.002954(8) \\
0.000398 \\
0.000000 \\
-0.005025 \\
0.003960 \\
0.000000 \\
-0.000557(3) \\
-0.001551 \\
-30.809045(9)\end{array}$ & $\begin{array}{c}-24.178633 \\
-0.006623 \\
-0.618060 \\
-7.605767(8) \\
-0.000664 \\
-3.57708(1) \\
-0.004271 \\
0.003646 \\
0.000000 \\
-0.000557(3) \\
-0.001551 \\
-35.98956(2)\end{array}$ & $\begin{array}{c}-24.178633 \\
-0.006623 \\
-0.618060 \\
-8.487013(8) \\
0.000280 \\
0.000000 \\
-0.003677 \\
0.003293 \\
0.000000 \\
-0.000557(3) \\
-0.001551 \\
-33.292541(9)\end{array}$ \\
\hline Term & $10^{1} M_{9}$ & $10^{3} M_{8}$ & $10^{3} M_{9}$ & $10^{3} M_{10}$ \\
\hline $\begin{array}{l}\Delta E_{\mathrm{nr}} \\
\Delta E_{\mathrm{M}}(1) \\
\Delta E_{\mathrm{M}}(2) \\
\Delta E_{\mathrm{rel}} \\
\Delta E_{\mathrm{anom}} \\
\Delta E_{\mathrm{st}} \\
\left(\Delta E_{\mathrm{RR}}\right)_{\mathrm{M}} \\
\left(\Delta E_{\mathrm{RR}}\right) \mathrm{X} \\
\Delta E_{\mathrm{nuc}} \\
\Delta E_{\mathrm{L}, 1} \\
\Delta E_{\mathrm{L}, 2} \\
\text { Total }\end{array}$ & $\begin{array}{c}-12.727808 \\
-0.003488 \\
-0.618059 \\
-5.297028(3) \\
0.000000 \\
2.868615(6) \\
-0.003380 \\
0.002715 \\
0.000000 \\
-0.000285(2) \\
-0.001110 \\
-15.779827(7)\end{array}$ & $\begin{array}{c}-12.727808 \\
-0.003488 \\
-0.618059 \\
-4.152161(3) \\
0.000280 \\
0.000000 \\
-0.003975 \\
0.002995 \\
0.000000 \\
-0.000285(2) \\
-0.001110 \\
-17.503610(3)\end{array}$ & $\begin{array}{c}-12.727808 \\
-0.003488 \\
-0.618059 \\
-5.399463(3) \\
-0.000475 \\
-2.868615(6) \\
-0.003380 \\
0.002743 \\
0.000000 \\
-0.000285(2) \\
-0.001110 \\
-21.619941(7)\end{array}$ & $\begin{array}{c}-12.727808 \\
-0.003488 \\
-0.618059 \\
-6.131145(3) \\
0.000204 \\
0.000000 \\
-0.002898 \\
0.002462 \\
0.000000 \\
-0.000285(2) \\
-0.001110 \\
-19.482127(3)\end{array}$ \\
\hline
\end{tabular}


TABLE XXIII. Comparison of theory (including $\Delta V_{\mathrm{ret}}^{\prime \prime}$ ) and experiment for the spin-averaged transition frequencies among the $n=10$ states of helium (in MHz).

\begin{tabular}{|c|c|c|c|c|c|}
\hline Transition & Experiment & Theory & Difference & $\Delta V_{\mathrm{ret}}^{\prime \prime}$ & $\Delta E_{D}^{(4)}$ \\
\hline$D-F$ & $14560.651(34)^{b}$ & $14560.6523(18)$ & $0.001(35)$ & -0.002397 & 0.000153 \\
\hline$F-G$ & $2036.5590(22)^{c}$ & $2036.57325(40)$ & $-0.0143(23)$ & -0.001223 & 0.000046 \\
\hline$G-H$ & $491.00523(49)^{c}$ & $491.00751(7)$ & $-0.00228(49)$ & -0.000714 & 0.000018 \\
\hline$H-I$ & $157.05241(23)^{c}$ & $157.05323(5)$ & $-0.00082(23)$ & -0.000453 & 0.000009 \\
\hline$I-K$ & $60.81595(20)^{\mathrm{c}}$ & $60.816471(14)$ & $-0.00052(20)$ & -0.000304 & 0.000004 \\
\hline$K-L$ & $27.17472(52)^{\mathrm{c}}$ & $27.175706(10)$ & $-0.00099(52)$ & -0.000213 & 0.000002 \\
\hline
\end{tabular}

${ }^{\mathrm{a}} \mathrm{Au}$ and Mesa [11].

${ }^{b}$ Farley, MacAdam, and Wing [60] global fit.

${ }^{\mathrm{c}}$ Hessels et al. [1].

known $10 D-10 F$ transition, will shed additional light on the subject, and ultimately lead to a confirmation of the Casimir-Polder effect.

\section{ACKNOWLEDGMENTS}

We are grateful to Richard Drachman and Eric Hessels for communicating the results of their asymptotic expansions in advance of publication, and to Stephen Lundeen for stimulating conversations. Research support by the Natural Sciences and Engineering Research Council of Canada, and by the Killam Foundation, is gratefully acknowledged.

\section{APPENDIX: ASYMPTOTIC RELATIONSHIPS AMONG SPIN-DEPENDENT RECOIL CORRECTIONS}

This appendix derives a number of useful asymptotic relationships among the relativistic-recoil corrections to the spin-dependent terms in the Breit interaction. It also demonstrates that the same results can be obtained either by a direct perturbation calculation involving the masspolarization operator, or by a transformation to Jacobi coordinates. The results are obtained in detail for $B_{3, Z}$ and $\Delta_{3}$. An exactly parallel transformation to Jacobi coordinates gives the corresponding asymptotic limits for $B_{3, e}$ shown in Eqs. (106) and (107).

Applying the Jacobi transformation $(3)-(5)$ to $B_{3, Z}$ yields immediately

$$
\begin{aligned}
B_{3, Z}= & \frac{Z \alpha^{2}}{2}\left(\frac{1}{r^{3}}\left(\mathbf{r} \times \mathbf{p}_{r}\right) \cdot \mathbf{s}_{1}+\frac{1}{|\mathbf{x}+y \mathbf{r}|^{3}}\left(\mathbf{x} \times \mathbf{p}_{x}\right) \cdot \mathbf{s}_{2}\right) \\
& +\frac{Z \alpha^{2}}{2} y\left(-\frac{1}{r^{3}}\left(\mathbf{r} \times \mathbf{p}_{x}\right) \cdot \mathbf{s}_{1}+\frac{1}{x^{3}}\left(\mathbf{x} \times \mathbf{p}_{x}\right) \cdot \mathbf{s}_{2}\right),
\end{aligned}
$$

up to terms linear in $y=\mu / M$. With use of the expansion

$$
\frac{1}{|\mathbf{x}+y \mathbf{r}|^{3}}=\frac{1}{x^{3}}\left(1-\frac{3 y}{x^{2}} \mathbf{r} \cdot \mathbf{x}+\cdots\right)
$$

and $(\mathbf{r} \cdot \mathbf{x})_{\mathrm{av}}=0$, the first line of (A1) reduces to the lowest-order spin-orbit interaction, and the second line is the recoil correction $B$ X, $Z$ linear in $y$. In the asymptotic approximation $x \gg r$, only the first term of the second line contributes. Comparison with Eq. (48) gives immediately

$$
\Delta_{3} \rightarrow-2 y B_{3, Z}^{\mathrm{X}}
$$

in conformity with the asymptotic limits displayed in Eqs. (106) and (107).

The alternative perturbation calculation proceeds as follows. Denoting the screened hydrogenic wave function for an infinite nuclear mass $1 s n L$ state by $\Psi_{\mathrm{SH}}$ and the mass-polarization correction by $\delta \Psi$, the recoil correction to $\left\langle B_{3, Z}\right\rangle$ is

$$
\left\langle B_{3, Z}^{\mathrm{X}}\right\rangle=\left\langle\Psi_{\mathrm{SH}}\left|B_{3, Z}\right| \delta \Psi\right\rangle+\left\langle\delta \Psi\left|B_{3, Z}\right| \Psi_{\mathrm{SH}}\right\rangle,
$$

where, in terms of spectral representations,

$$
\left\langle\delta \Psi\left|B_{3, Z}\right| \Psi_{\mathrm{SH}}\right\rangle=y \sum_{\substack{n^{\prime}, l^{\prime} \\ n^{\prime \prime}, l^{\prime \prime}}} \frac{\left\langle\Psi_{\mathrm{SH}}\left|\mathbf{p}_{1} \cdot \mathbf{p}_{2}\right| n^{\prime} l^{\prime}, n^{\prime \prime} l^{\prime \prime}\right\rangle\left\langle n^{\prime} l^{\prime}, n^{\prime \prime} l^{\prime \prime}\left|B_{3, Z}\right| \Psi_{\mathrm{SH}}\right\rangle}{E(1 s, n l)-E\left(n^{\prime} l^{\prime}, n^{\prime \prime} l^{\prime \prime}\right)}
$$

With use of

$$
\mathbf{p}_{r}=i\left[h_{r}(\mathbf{Z}), \mathbf{r}\right],
$$

where $h_{r}(Z)$ is the one-electron Hamiltonian for the unscreened inner electron with coordinate $\mathbf{r}$ and

$$
E(1 s, n l)-E\left(n^{\prime} l^{\prime}, n^{\prime \prime} l^{\prime \prime}\right) \simeq E(1 s)-E\left(n^{\prime} l^{\prime}\right)
$$

in the adiabatic approximation, the commutator approxi- mately cancels the energy difference in the denominator of (A5). The sums over intermediate states can then be completed by closure. Combining the two terms in (A4) gives

$$
\left\langle B_{3, Z}^{\mathrm{X}}\right\rangle=y\left\langle\Psi_{\mathrm{SH}}\left|\left[\mathbf{r}_{1} \cdot \nabla_{2}, B_{3, Z}\right]\right| \Psi_{\mathrm{SH}}\right\rangle
$$

Evaluation of the commutator gives the final result 


$$
\begin{gathered}
\left\langle B_{3, Z}^{\mathrm{X}}\right\rangle=\frac{1}{2} Z \alpha^{2} y\left\langle\Psi_{\mathrm{SH}}\right|-\frac{1}{r^{3}}\left(\mathbf{r} \times \mathbf{p}_{x}\right) \cdot \mathbf{s}_{1}+\frac{1}{x^{3}}\left(\mathbf{r} \times \mathbf{p}_{x}\right) \cdot \mathbf{s}_{2} \\
-\frac{3 \mathbf{r} \cdot \mathbf{x}}{x^{5}}\left(\mathbf{r} \times \mathbf{p}_{x}\right) \cdot \mathbf{s}_{2}\left|\Psi_{\mathrm{SH}}\right\rangle
\end{gathered}
$$

in agreement with the corresponding terms from (A1) obtained from the Jacobi transformation.

The expectation value $\left\langle\Delta_{3}\right\rangle$ vanishes in any oneelectron approximation because of the symmetry of the $\mathbf{r} \cdot \mathbf{p}_{x}$ operator. The leading nonvanishing contribution comes from perturbations to the screened hydrogenic wave function due to the dipole term

$$
V_{1}=\frac{r}{x^{2}} \cos (\hat{\mathbf{r}} \cdot \hat{\mathbf{x}})
$$

in Eq. (11). Denoting the wave-function correction by $\delta \Psi^{\prime}$, the matrix element is

$$
\left\langle\Delta_{3}\right\rangle=\left\langle\Psi_{\mathrm{SH}}\left|\Delta_{3}\right| \delta \Psi^{\prime}\right\rangle+\left\langle\delta \Psi^{\prime}\left|\Delta_{3}\right| \Psi_{\mathrm{SH}}\right\rangle,
$$

where, in parallel with Eq. (A5),

$$
\begin{aligned}
& \left\langle\delta \Psi^{\prime}\left|\Delta_{3}\right| \Psi_{\mathrm{SH}}\right\rangle \\
& =\sum_{\substack{n^{\prime}, l^{\prime} \\
n^{\prime \prime}, l^{\prime \prime}}} \frac{\left\langle\Psi_{\mathrm{SH}}\left|V_{1}\right| n^{\prime} l^{\prime}, n^{\prime \prime} l^{\prime \prime}\right\rangle\left\langle n^{\prime} l^{\prime}, n^{\prime \prime} l^{\prime \prime}\left|\Delta_{3}\right| \Psi_{\mathrm{SH}}\right\rangle}{E(1 s, n l)-E\left(n^{\prime} l^{\prime}, n^{\prime \prime} l^{\prime \prime}\right)} .
\end{aligned}
$$

With use of

$$
Z \frac{\mathbf{r}}{r^{3}}=i\left[\mathbf{p}_{r}, h_{r}(Z)\right]
$$

to replace the corresponding factors in the dominant $r^{-3}$ part of $\Delta_{3}$, the same steps as those leading to Eq. (A8) yield

$$
\left\langle\Delta_{3}\right\rangle \rightarrow-i \alpha^{2} y\left\langle\Psi_{\mathrm{SH}}\left|\mathbf{s}_{1} \cdot\left[\mathbf{p}_{r} \times \mathbf{p}_{x}, V_{1}\right]\right| \Psi_{\mathrm{SH}}\right\rangle
$$

in the adiabatic approximation. Evaluation of the commutator and discarding terms proportional to $\mathbf{r} \times \mathbf{p}_{r}$, $\mathbf{r} \times \mathbf{p}_{x}$, and $\mathbf{x} \times \mathbf{p}_{r}$ that vanish for $1 s n l$ states gives

$$
\left\langle\Delta_{3}\right\rangle \rightarrow-\alpha^{2} \frac{\mu}{M}\left\langle\Psi_{\mathrm{SH}}\left|\frac{1}{x^{3}}\left(\mathbf{x} \times \mathbf{p}_{x}\right) \cdot \mathbf{s}_{1}\right| \Psi_{\mathrm{SH}}\right\rangle .
$$

Comparing with Eq. (34) and using $\mathbf{r}_{1} \simeq \mathbf{r}, \mathbf{r}_{2} \simeq \mathbf{x}$, it is evident that for diagonal triplet matrix elements

$\left\langle 1 s n l{ }^{3} L\left|\Delta_{3}\right| 1 s n l{ }^{3} L\right\rangle$

$$
\rightarrow-2 Z^{-1} y\left\langle 1 s n l^{3} L\left|B_{3, Z}\right| 1 s n l^{3} L\right\rangle,
$$

and for off-diagonal matrix elements

$$
\begin{aligned}
& \left\langle 1 s n l{ }^{3} L\left|\Delta_{3}\right| 1 s n l{ }^{1} L\right\rangle \\
& \quad \rightarrow 2 Z^{-1} y\left\langle 1 s n l^{3} L\left|B_{3, Z}\right| 1 s n l{ }^{1} L\right\rangle
\end{aligned}
$$

in agreement with Eqs. (106) and (107).
[1] E. A. Hessels, F. J. Deck, P. W. Arcuni, and S. R. Lundeen, Phys. Rev. Lett. 65, 2765 (1990); 66, 2544(E) (1991); Phys. Rev. A (to be published).

[2] S. R. Lundeen, in Long Range Forces: Theory and Recent Experiments in Atomic Systems, edited by F. S. Levin and D. Micha (Plenum, New York, 1992).

[3] G. W. F. Drake, J. Phys. B 22, L651 (1989); 23, 1943(E) (1990).

[4] G. W. F. Drake, Phys. Rev. Lett. 65, 2769 (1990).

[5] G. W. F. Drake, in Long Range Forces: Theory and Recent Experiments in Atomic Systems, edited by F. S. Levin and D. Micha (Plenum, New York, 1992).

[6] S. P. Goldman and G. W. F. Drake, Phys. Rev. Lett. 68, 1683 (1992).

[7] E. J. Kelsey and L. Spruch, Phys. Rev. A 18, 15 (1978); 18, 1055 (1978).

[8] C.-K. Au, G. Feinberg, and J. Sucher, Phys. Rev. Lett. 53, 1145 (1984).

[9] J. F. Babb and L. Spruch, Phys. Rev. A 38, 13 (1988).

[10] C.-K. Au, Phys. Rev. A 39, 2789 (1989).

[11] C.-K. Au and M. A. Mesa, Phys. Rev. A 41, 2848 (1990).

[12] L. Spruch, in Long Range Forces: Theory and Recent Experiments in Atomic Systems, edited by F. S. Levin and D. Micha (Plenum, New York, 1992).

[13] See, for example, A. I. Akhiezer and V. B. Berestetskii, Quantum Electrodynamics (Interscience, New York, 1965), Sec. 38.

[14] A. Dalgarno and R. W. McCarroll, Proc. R. Soc. London Ser. A 237, 383 (1956).

[15] A. Dalgarno, G. W. F. Drake, and G. A. Victor, Phys. Rev. 176, 194 (1968).
[16] C. J. Kleinman, Y. Hahn, and L. Spruch, Phys. Rev. 165, 53 (1968).

[17] C. Deutsch, Phys. Rev. A 2, 43 (1970); 3, 1516(E) (1971); 13, 2311 (1976).

[18] R. J. Drachman, Phys. Rev. A 26, 1228 (1982).

[19] R. J. Drachman, Phys. Rev. A 31, 1253 (1985); 38, 1659(E) (1988).

[20] R. J. Drachman, Phys. Rev. A 33, 2780 (1986); 37, 979 (1988).

[21] R. J. Drachman, in Long Range Forces: Theory and Recent Experiments in Atomic Systems, edited by F. S. Levin and D. Micha (Plenum, New York, 1992).

[22] G. W. F. Drake, Phys. Rev. Lett. 59, 1549 (1987).

[23] G. W. F. Drake, Nucl. Instrum. Methods Phys. Res. Sect. B 31, 7 (1988).

[24] G. W. F. Drake and A. J. Makowski, J. Opt. Soc. Am. B 5, 2207 (1988).

[25] H. A. Bethe and E. E. Salpeter, Quantum Mechanics of One- and Two-Electron Atoms (Springer-Verlag, Berlin, 1957).

[26] G. W. F. Drake, Adv. At. Mol. Phys. (to be published).

[27] G. W. F. Drake and R. A. Swainson, Phys. Rev. A 44, 5448 (1991).

[28] R. A. Swainson and G. W. F. Drake, Can. J. Phys. 70, 187 (1992).

[29] A. P. Stone, Proc. Phys. Soc. (London) 77, 786 (1961); 81, 868 (1963); M. Douglas and N. M. Kroll, Ann. Phys. (N.Y.) 62, 89 (1974).

[30] A. M. Sessler and H. M. Foley, Phys. Rev. 92, 1321 (1953); 98, 6 (1955).

[31] M. L. Lewis and P. H. Serafino, Phys. Rev. A 18, 867 
(1978).

[32] E. A. Hessels (unpublished).

[33] A. Dalgarno and J. T. Lewis, Proc. R. Soc. London Ser. A 233, 70 (1956); A. Dalgarno and A. L. Stewart, ibid. 238, 269 (1956).

[34] C.-K. Au, G. Feinberg, and J. Sucher, Phys. Rev. A 43, 561 (1991).

[35] G. W. F. Drake, Phys. Rev. A 45, 70 (1992); 45, 6933(E) (1992).

[36] E. A. Hessels, W. G. Sturrus, S. R. Lundeen, and D. R. Cok, Phys. Rev. A 35, 4489 (1987).

[37] R. J. Boyd and C. A. Coulson, J. Phys. B 6, 782 (1973).

[38] H. Araki, Prog. Theor. Phys. 17, 619 (1957).

[39] J. Sucher, Phys. Rev. 109, 1010 (1958).

[40] D. R. Cok and S. R. Lundeen, Phys. Rev. A 19, 1830 (1979); 24, 3283(E) (1981).

[41] T. N. Chang, Phys. Rev. A 39, 6129 (1989).

[42] M. Idrees and C. F. Fischer, Nucl. Instrum. Methods Phys. Res. B 42, 552 (1989).

[43] C. Schwartz, Phys. Rev. 134, A1181 (1964).

[44] M. Douglas and N. M. Kroll, Ann. Phys. (N.Y.) 82, 89 (1974).

[45] E. R. Cohen and B. N. Taylor, Rev. Mod. Phys. 59, 1121 (1987); Sec. B 10.

[46] G. W. Erickson and D. R. Yennie, Ann. Phys. (N.Y.) 35, 271 (1965); 35, 447 (1965).

[47] G. W. F. Drake, Adv. At. Mol. Phys. 18, 399 (1982).
[48] J. R. Sapirstein and D. R. Yennie, in Quantum Electrodynamics, edited by T. Kinoshita (World Scientific, Singapore, 1990).

[49] E. Borie and G. A. Rinker, Phys. Rev. A 18, 324 (1978). See also A. van Wijngaarden, J. Kwela, and G. W. F. Drake, Phys. Rev. A 43, 3325 (1991), Sec. VI for a recent discussion of nuclear radius measurements.

[50] G. W. F. Drake and R. A. Swainson, Phys. Rev. A 41, 1243 (1990).

[51] S. P. Goldman and G. W. F. Drake, J. Phys. B 16, L183 (1983); 17, L197 (1984).

[52] S. P. Goldman, Phys. Rev. A 30, 1219 (1984).

[53] J. Baker, G. C. Forrey, R. N. Hill, M. Jerziorska, J. D. Morgan III, and J. Shertzer (unpublished).

[54] W. Lichten, D. Shiner, and Z.-X. Zhao, Phys. Rev. A 43, 1663 (1991).

[55] L. Hlousek, S. A. Lee, and W. M. Fairbank, Jr., Phys. Rev. Lett. 50, 328 (1983).

[56] B. Edlén, in Encyclopedia of Physics (Springer, Berlin, 1964), Vol. XXVII.

[57] G. W. F. Drake and R. A. Swainson, Phys. Rev. A 44, 5448 (1991).

[58] C. J. Sansonetti and J. D. Gillaspy, Phys. Rev. A 45, R1 (1992).

[59] G. W. F. Drake, Adv. At. Mol. Phys. (to be published).

[60] J. W. Farley, K. B. McAdam, and W. H. Wing, Phys. Rev. A 20, 1754 (1979); 25, 1790(E) (1982). 